\title{
INTEGRAL MOMENTS OF L-FUNCTIONS
}

\author{
J. B. CONREY, D. W. FARMER, J. P. KEATING, M. O. RUBINSTEIN, N. C. SNAITH
}

\begin{abstract}
We give a new heuristic for all of the main terms in the integral moments of various families of primitive $L$-functions. The results agree with previous conjectures for the leading order terms. Our conjectures also have an almost identical form to exact expressions for the corresponding moments of the characteristic polynomials of either unitary, orthogonal, or symplectic matrices, where the moments are defined by the appropriate group averages. This lends support to the idea that arithmetical $L$-functions have a spectral interpretation, and that their value distributions can be modeled using Random Matrix Theory. Numerical examples show good agreement with our conjectures.
\end{abstract}

\section{Contents}

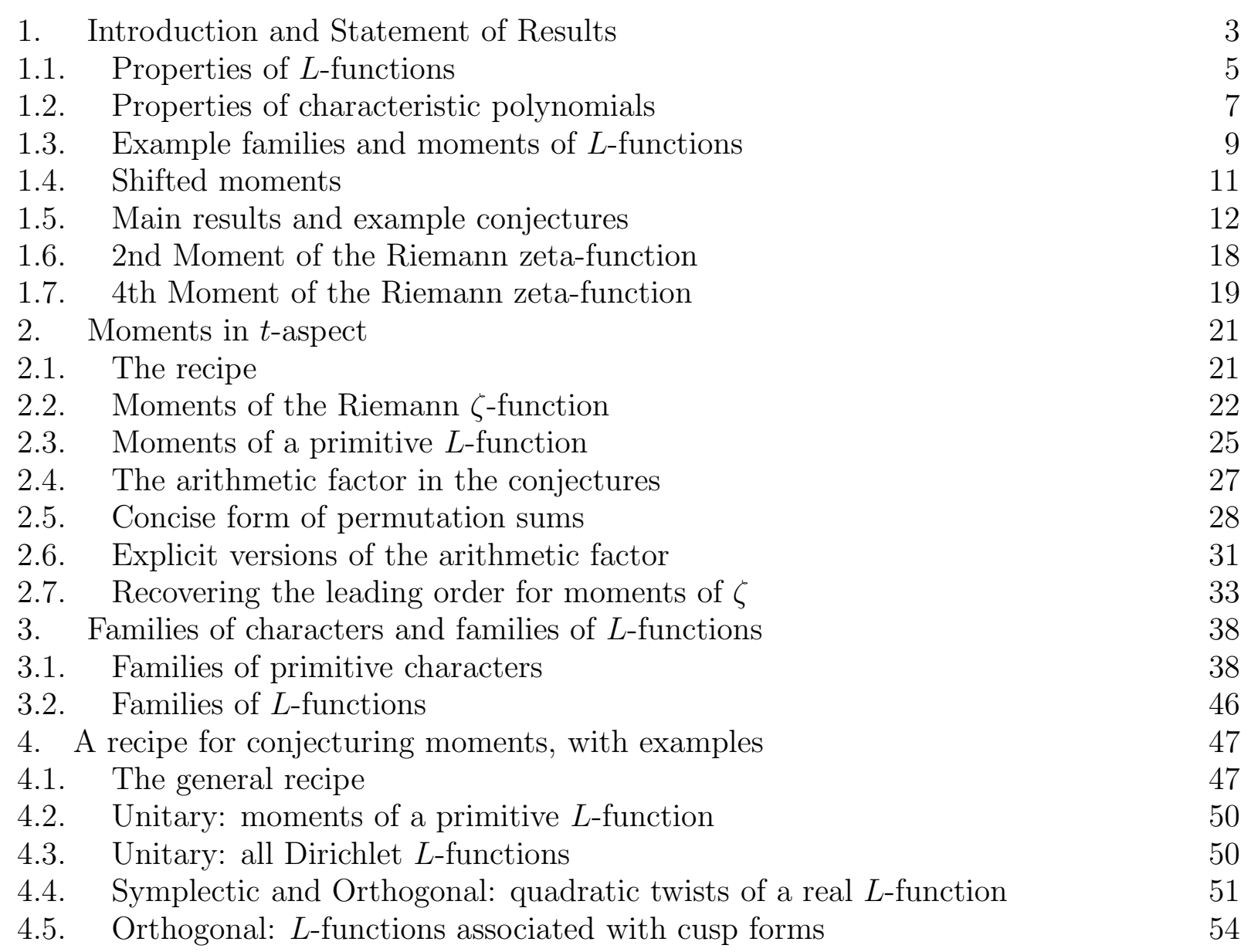

Research partially supported by the American Institute of Mathematics and a Focused Research Group grant from the National Science Foundation. The last author was also supported by a Royal Society Dorothy Hodgkin Fellowship. 
5. Numerical calculations

5.1. Unitarv: Riemann zeta-function

5.2. Svmplectic: quadratic Dirichlet $L$-functions

5.3. Orthogonal: twists of a $G L(2) L$-function

59

References 


\section{Introduction and Statement of Results}

Random Matrix Theory (RMT) has recently become a fundamental tool for understanding $L$-functions. Montgomery [Mon] showed that the two-point correlations between the non-trivial zeros of the Riemann $\zeta$-function, on the scale of the mean zero spacing, are similar to the corresponding correlations between the eigenvalues of random unitary matrices in the limit of large matrix size [Meh] and conjectured that these correlations are, in fact, identical to each other. There is extensive numerical evidence Odl in support of this conjecture. Rudnick and Sarnak RS extended Montgomery's analysis to all $n$-point correlations, and to the zeros of other principal $L$-functions. Katz and Sarnak [KSa] introduced the idea of studying zero distributions within families of $L$-functions (see also [OS, Rub]) and have conjectured that these coincide with the eigenvalue distributions of the classical compact groups. In this context symmetries of an $L$-function family determine the associated classical group. We shall here be concerned with the distribution of values taken by $L$-functions, either individually (i.e. along the appropriate critical line), or with respect to averages over families. Specifically, we shall calculate the integral moments of these distributions.

Keating and Snaith [KS1] suggested that the value distribution of the Riemann $\zeta$-function (or any other principal $L$-function) on its critical line is related to that of the characteristic polynomials of random unitary matrices. This led them to a general conjecture for the leading-order asymptotics of the moments of this distribution in the limit of large averaging range. Their conjecture agrees with a result of Hardy and Littlewood [HL for the second moment and a result of Ingham [I] for the fourth moment (see, for example [T]). It also agrees with conjectures, based on number-theoretical calculations, of Conrey and Ghosh [CG2] and Conrey and Gonek [CGo for the sixth and eighth moments. General conjectures for the leading-order asymptotics of the moments of $L$-functions within families, based on randommatrix calculations for the characteristic polynomials of matrices from the orthogonal and unitary-symplectic groups, were developed by Conrey and Farmer [CF] and Keating and Snaith KS2]. These are also in agreement with what is known, and with previous conjectures.

Our purpose here is, for the integral moments of a family of primitive $L$-functions, to go beyond the leading order asymptotics previously investigated: we give conjectures for the full main terms. We propose a refined definition of "conductor" of an $L$-function, which to leading order is the (logarithm of) the "usual" conductor. We find that often, but not always, the mean values can be expressed as polynomials in the conductor. Importantly, our conjectures show a striking formal similarity with analogous expressions for the characteristic polynomials of random matrices. This provides a strong measure of the depth of the connection between $L$-functions and RMT. We also perform numerical calculations which show very good agreement with our conjectures. Non-primitive families can also be handled by our methods, but we do not treat those here.

The conjectures we develop here can also be obtained by techniques of multiple Dirichlet series, as described by Diaconu, Goldfeld, and Hoffstein DGH. In their formulation, one considers Dirichlet series in several complex variables. The mean values we conjecture would then follow from a plausible conjecture about the polar divisors of the function. An interesting feature of their approach is that for higher moments it seems to predict lower order terms of the form $c T^{A}$ with $\frac{1}{2}<A<1$, while in this paper we conjecture that our main terms are valid with an error of size $O\left(T^{\frac{1}{2}+\varepsilon}\right)$. The cubic moment of quadratic Dirichlet $L$-functions is 
a specific case for which there is a conjectured lower order term [Z] which possibly could be tested numerically.

There are many theorems dealing with moments of $L$-functions in particular families. The technique to prove these theorems usually involves invoking an approximate functional equation and averaging the coefficients of the $L$-function over the family. The averaging process behaves like a harmonic detection device. This harmonic detector usually presents itself as a formula with a relatively simple part and a somewhat more complicated part that is smaller in the first approximation. In the theorems in the literature it is often the case that the simple part of the harmonic detector is sufficiently good to determine the first or second moment of the family. The terms involved here are usually called the "diagonal" terms. But invariably the more complicated version is needed to determine the asymptotics of the third or fourth moments; in these situations one has gone "beyond the diagonal." In at least one situation (fourth moment of cusp form $L$-functions) it has been necessary to identify three separate stages of more subtle harmonic detection: the first featuring diagonal term contributions and the second and third featuring contributions to the main terms by two different types of off-diagonal terms. We believe that as one steps up the moments of a family then at every one or two steps a new type of off-diagonal contribution will emerge. The whole process is poorly understood; we only have glimpses of a mechanism but no clear idea of how or why it works.

It is remarkable that all of these complicated harmonic detection devices ultimately lead to very simple answers, as detailed in this paper. It is also remarkable that there are only three or four different types of symmetries; families with the same symmetry type often have different harmonic detectors, with different wrinkles at each new stage of off-diagonal, but somehow lead to answers which are structurally the same. It would be worthwhile to understand how this works.

Finally, we comment that the recipe we develop in this paper only uses the simplest diagonal harmonic detectors. Our formulas are expressed as combinatorial sums arising only from diagonal terms. We are well aware of the off-diagonal pieces, and we do not understand how they cancel and combine. What we do understand and what we are presenting here is a conjecture for the final simple answer that should emerge after all of the complicated cancellations between the increasingly subtle off-diagonal terms are taken into account. The reader needs to be aware of this to understand the goals and contents of this paper.

The paper is organized as follows. In the remainder of this section we give a detailed comparison between $L$-functions and characteristic polynomials of unitary matrices, summarize our previous work on the leading terms in the mean values of $L$-functions, and describe the more general moments considered in this paper. This allows us to state our main results and conjectures, which are given in Section [1.5. We then give a detailed comparison with known results for the Riemann $\zeta$-function.

In Section 2 we give a detailed derivation of our conjectures in the case of moments on the critical line of a single $L$-function. We first write the conjecture in terms of a function defined by an infinite sum, and then write it as an Euler product and identify the leading-order poles. The local factors are also written in a concise form which is more suitable for computation. Both the $L$-function and random matrix calculations lead to expressions involving a sum over a set of partitions. These sums can be written in a concise form involving contour integrals, as described in Section 2.5. We also show that the original results of Keating and 
Snaith [KS1, KS2] for the leading order term can be re-derived from the present work. In addition, we express the arithmetic factor in the moments of the Riemann zeta-function in an explicit form.

In Section 3 we describe a particular notion of a family of $L$-functions which can be used to give a unified treatment of all of the mean values we have considered. These families are central to our method of conjecturing mean values and we give a detailed description of the method in Section 4. As explicit examples we give the details of the calculations for $L$-function families with Unitary, Symplectic, and Orthogonal symmetry.

In Section 5 we give numerical approximations for the coefficients in our conjectured mean values. We then report on numerical calculations of representative cases of the conjectures. Good agreement is found.

The calculations of the random matrix averages, which are based in part on $[\mathrm{BF}$ and $[\mathrm{BH}]$, are complicated but elementary. Those results have been presented in [CFKRS]. In subsequent papers we will also present a fuller discussion of the terms which appear in our conjectures, give some more general conjectures, and describe the algorithms behind our numerical calculations.

The authors thank P. Forrester, R. Heath-Brown, C. Hughes, N. Katz, P. Michel, and P. Sarnak for many helpful discussions.

1.1. Properties of $L$-functions. We present the definition and key properties of $L$-functions. These properties are familiar, but a summary will be useful in our discussion of mean values and for the comparison with the characteristic polynomials of random matrices.

The definition of an $L$-function which we give below is a slight modification of what has come to be called the "Selberg class" [S, CG3, Mur] of Dirichlet series. Let $s=\sigma+i t$ with $\sigma$ and $t$ real. An $L$-function is a Dirichlet series

$$
L(s)=\sum_{n=1}^{\infty} \frac{a_{n}}{n^{s}},
$$

with $a_{n} \ll_{\varepsilon} n^{\varepsilon}$ for every $\varepsilon>0$, which has three additional properties.

Analytic continuation: $L(s)$ continues to a meromorphic function of finite order with at most finitely many poles, and all poles are located on the $\sigma=1$ line.

Functional equation: There is a number $\varepsilon$ with $|\varepsilon|=1$, and a function $\gamma_{L}(s)$ of the form

$$
\gamma_{L}(s)=P(s) Q^{s} \prod_{j=1}^{w} \Gamma\left(w_{j} s+\mu_{j}\right),
$$

where $Q>0, w_{j}>0, \Re \mu_{j} \geq 0$, and $P$ is a polynomial whose only zeros in $\sigma>0$ are at the poles of $L(s)$, such that

$$
\xi_{L}(s):=\gamma_{L}(s) L(s)
$$

is entire, and

$$
\xi_{L}(s)=\varepsilon \overline{\xi_{L}}(1-s),
$$

where $\overline{\xi_{L}}(s)=\overline{\xi_{L}(\bar{s})}$ and $\bar{s}$ denotes the complex conjugate of $s$. 
The number $2 \sum_{j=1}^{w} w_{j}$ is called the degree of the $L$-function, and this is conjectured to be an integer. It is conjectured furthermore that each $w_{j}$ can be taken to equal $\frac{1}{2}$, so $w$ equals the degree of the $L$-function.

For the calculations we do in this paper, it is convenient to write the functional equation in asymmetric form:

$$
L(s)=\varepsilon X_{L}(s) \bar{L}(1-s),
$$

where $X_{L}(s)=\frac{\overline{\gamma_{L}}(1-s)}{\gamma_{L}(s)}$. Also we define the " $Z$-function" associated to an $L$-function:

$$
Z_{L}(s):=\varepsilon^{-\frac{1}{2}} X_{L}^{-\frac{1}{2}}(s) L(s),
$$

which satisfies the functional equation

$$
Z_{L}(s)=\overline{Z_{L}}(1-s) .
$$

Note that here we define $Z_{L}$ as a function of a complex variable, which is slightly different from the standard notation. Note also that $Z_{L}\left(\frac{1}{2}+i t\right)$ is real when $t$ is real, $X_{L}\left(\frac{1}{2}\right)=1$, and $\left|X_{L}\left(\frac{1}{2}+i t\right)\right|=1$ if $t$ is real.

Euler product: For $\sigma>1$ we have

$$
L(s)=\prod_{p} L_{p}\left(1 / p^{s}\right)
$$

where the product is over the primes $p$, and

$$
L_{p}\left(1 / p^{s}\right)=\sum_{k=0}^{\infty} \frac{a_{p^{k}}}{p^{k s}}=\exp \left(\sum_{k=1}^{\infty} \frac{b_{p^{k}}}{p^{k s}}\right),
$$

where $b_{n} \ll n^{\theta}$ with $\theta<\frac{1}{2}$.

Note that $L(s) \equiv 1$ is the only constant $L$-function, the set of $L$-functions is closed under products, and if $L(s)$ is an $L$-function then so is $L(s+i y)$ for any real $y$. An $L$ function is called primitive if it cannot be written as a nontrivial product of $L$-functions, and it can be shown, assuming Selberg's orthonormality conjectures, that any $L$-function has a unique representation as a product of primitive $L$-functions. See [CG3. It is believed that $L$-functions only arise from arithmetic objects, such as characters [Dav], automorphic forms [Iw1, Iw2, and automorphic representations $\mathrm{BC}, \mathrm{Bu}$. Very little is known about $L$-functions beyond those cases which have been shown to be arithmetic.

There are several interesting consequences of the above properties, and many conjectures which have been established in a few (or no) cases. We highlight some additional properties of $L$-functions and then discuss their random matrix analogues.

Location of zeros: Since $\xi_{L}(s)$ is entire, $L(s)$ must vanish at the poles of the $\Gamma$-functions in the $\gamma_{L}$ factor. These are known as the trivial zeros of the $L$-function. By the functional equation and the Euler product, the only other possible zeros of $L(s)$ lie in the critical strip $0 \leq \sigma \leq 1$. By the argument principle, the number of nontrivial zeros with $0<t<T$ is asymptotically $(W / \pi) T \log T$, where $W=\sum w_{j}$. The Riemann Hypothesis for $L(s)$ asserts that the nontrivial zeros of $L(s)$ lie on the critical line $\sigma=\frac{1}{2}$. The much weaker (but still deep) assertion that $L(s) \neq 0$ on $\sigma=1$ has been proven for arithmetic $L$-functions [JS], which can be viewed as a generalization of the prime number theorem. 
Average spacing of zeros: By the zero counting result described above, the average gap between consecutive zeros of $L(s)$ with imaginary part around $T$ is $\pi /(W \log T)$.

Zeros of derivatives: If the Riemann Hypothesis is true then all zeros of the derivative $\xi^{\prime}(s)$ lie on the critical line, while all zeros of $\zeta^{\prime}(s)$ lie to the right of the critical line [LM].

Critical values: The value $L\left(\frac{1}{2}\right)$ is called the critical value of the $L$-function. The significance of $s=\frac{1}{2}$ is that it is the symmetry point of the functional equation. The mean values we study in this paper are averages of (powers of) critical values of $L$-functions, where the average is taken over a "family" of $L$-functions. Examples of families and their corresponding mean values are given in Section 1.3 .

Note. If the set $\left\{\mu_{j}\right\}$ is stable under complex conjugation and the $a_{n}$ are real, then $\varepsilon$ is commonly called the sign of the functional equation. If the sign is -1 then $L(s)$ has an odd order zero at $s=\frac{1}{2}$; more generally, if the sign is not 1 then $L\left(\frac{1}{2}\right)=0$. When $L\left(\frac{1}{2}\right)$ vanishes, it is common to use the term 'critical value' for the first nonzero derivative $L^{(j)}\left(\frac{1}{2}\right)$, but in this paper we use 'critical value' to mean 'value at the critical point.'

Log conductor: We measure the "size" of an $L$-function by its log conductor, defined as $c(L)=\operatorname{cond}(L)=\left|X_{L}^{\prime}\left(\frac{1}{2}\right)\right|$. The conductor of an $L$-function has a conventional meaning in many contexts, and the log conductor is a simple function of the (logarithm of the) usual conductor. Other authors use similar names, such as "analytic conductor", for similar quantities. By the argument principle, the density of zeros near the critical point is $2 \pi c(L)^{-1}$.

Approximate functional equation: A standard tool for studying analytic properties of $L$ functions is an approximate functional equation for $L(s)$, which expresses the $L$-function as a sum of two Dirichlet series involving the Dirichlet coefficients of $L$ multiplied by a smoothing function. See for example [K, 5.3]. For the purposes of the heuristics we develop, we use a sharp cutoff and don't concern ourselves with the remainder,

$$
L(s)=\sum_{m<x} \frac{a_{m}}{m^{s}}+\varepsilon X_{L}(s) \sum_{n<y} \frac{\overline{a_{n}}}{n^{1-s}}+\text { remainder } .
$$

Here the product $x y$ depends on parameters in the functional equation. The name comes from the fact that the right side looks like $L(s)$ if $x$ is large, and like $\varepsilon X_{L}(s) \bar{L}(1-s)$ if $x$ is small, which suggests the asymmetric form of the functional equation.

The approximate functional equation is the starting point of our approach to conjecturing the moments of $L$-functions. This is described in Sections 2.1 and 4.1.

1.2. Properties of characteristic polynomials. With the exception of the Euler product, all of the properties of $L$-functions have a natural analogue in the characteristic polynomials of unitary matrices. We note each property in turn.

Let

$$
\Lambda(s)=\Lambda_{A}(s)=\operatorname{det}\left(I-A^{*} s\right)=\prod_{n=1}^{N}\left(1-s e^{-i \theta_{n}}\right)
$$

denote the characteristic polynomial of an $N \times N$ matrix $A$. Throughout the paper we assume that $A$ is unitary (i.e. $A^{*} A=I$ where $A^{*}$ is the Hermitian conjugate of $A$ ), so the eigenvalues of $A$ lie on the unit circle and can be denoted by $e^{i \theta_{n}}$. 
Note: in our previous paper CFKRS] we used a different definition of the characteristic polynomial.

We can express $\Lambda(s)$ in expanded form:

$$
\Lambda(s)=\sum_{n=0}^{N} a_{n} s^{n},
$$

which corresponds to the Dirichlet series representation for $L$-functions.

Analytic continuation: Since $\Lambda(s)$ is a polynomial, it is an entire function.

Functional equation: Since $A$ is unitary, we have

$$
\Lambda_{A}(s)=(-1)^{N} \operatorname{det} A^{*} s^{N} \operatorname{det}\left(I-A s^{-1}\right),
$$

and so, writing

$$
\operatorname{det} A=e^{i \phi}
$$

(where unitarity implies that $\phi \in \mathbb{R}$ ), we have

$$
\begin{aligned}
\Lambda_{A}(s) & =(-1)^{N} \operatorname{det} A^{*} s^{N} \Lambda_{A^{*}}\left(\frac{1}{s}\right) \\
& =(-1)^{N} e^{-i \phi} s^{N} \overline{\Lambda_{A}}\left(\frac{1}{s}\right)
\end{aligned}
$$

This plays the same role for $\Lambda(s)$ as the functional equation for $L$-functions: it represents a symmetry with respect to the unit circle $\left(s=r e^{i \alpha} \rightarrow \frac{1}{s}=\frac{1}{r} e^{-i \alpha}\right)$.

Also let

$$
\mathcal{Z}_{A}(s)=\left((-1)^{N} e^{-i \phi}\right)^{-\frac{1}{2}} s^{-N / 2} \Lambda_{A}(s)
$$

in direct analogy to (1.1.6), the sign of the functional equation $\varepsilon$ being identified with $(-1)^{N} e^{-i \phi}=(-1)^{N} \operatorname{det} A^{*}$. The functional equation becomes

$$
\mathcal{Z}_{A}(s)=\overline{\mathcal{Z}}_{A}\left(\frac{1}{s}\right) \text {. }
$$

Note that this implies $\mathcal{Z}$ is real on the unit circle, and in analogy to the $X_{L}$ factor from an $L$-function, the factor $s^{-N / 2}$ equals 1 at the critical point $s=1$, and has absolute value 1 on the unit circle.

Location of zeros: Since $A$ is unitary, its eigenvalues all have modulus 1 , so the zeros of $\Lambda(s)$ lie on the unit circle (i.e. the Riemann Hypothesis is true). The unit circle is the "critical line" for $\Lambda(s)$.

Average spacing of zeros: Since the $N \times N$ matrix $A$ has $N$ eigenvalues on the unit circle, the average spacing between zeros of $\Lambda_{A}(s)$ is $2 \pi / N$.

Zeros of derivatives: Since the zeros of $\Lambda(s)$ lie on the unit circle, the zeros of the derivative $\Lambda^{\prime}$ lie inside the unit circle. This follows from the general fact that the zeros of the derivative of a polynomial lie in the convex hull of the zeros of the polynomial.

Critical values: The critical point for $\Lambda(s)$ is the symmetry point of the functional equation $s=1=e^{i \cdot 0}$, and $\Lambda(1)$ is the critical value.

Conductor: In analogy with the case of $L$-functions, we define the conductor of $\Lambda$ to be the (absolute value of the) derivative of the factor in the asymmetric form of the functional equation, evaluated at the critical point $s=1$. That is, the conductor of $\Lambda$ is $N$. Also in analogy to the case of $L$-functions, the density of zeros on the unit circle is $2 \pi / N$. 
When modeling a family of $L$-functions, we choose $N$ so that $L$ and $\Lambda$ have the same conductor. Equivalently, $L$ and $\Lambda$ have the same density of zeros near the critical point.

Approximate functional equation: Substituting the polynomial (1.2.2) into the functional equation (1.2.5), we have

$$
\sum_{n=0}^{N} a_{n} s^{n}=(-1)^{N} e^{-i \phi} \sum_{n=0}^{N} \overline{a_{n}} s^{N-n},
$$

and so

$$
a_{n}=(-1)^{N} e^{-i \phi} \overline{a_{N-n}}
$$

Hence, when $N$ is odd, we have

$$
\Lambda(s)=\sum_{m=0}^{\frac{N-1}{2}} a_{m} s^{m}+(-1)^{N} e^{-i \phi} s^{N} \sum_{n=0}^{\frac{N-1}{2}} \overline{a_{n}} s^{-n},
$$

which corresponds to the approximate functional equation for $L$-functions. When $N$ is even, there is an additional term: $a_{\frac{N}{2}} s^{\frac{N}{2}}$.

Although we use the approximate functional equation in our calculations for $L$-functions, in our previous paper [CFKRS] we use other methods for the characteristic polynomials. In principle, it would be possible to use the approximate functional equation and compute averages of products of the coefficients $a_{n}$. Such a calculation would, presumably, mirror that for the $L$-functions. This would appear to be more cumbersome than the approach taken in CFKRS, but might merit further investigation.

The above discussion applies to any unitary matrix. We also consider matrices which, in addition to being unitary, are either symplectic or orthogonal. We use these three ensembles of matrices to model families of $L$-functions. While the notion of "family of $L$-functions" has not yet been made precise, we give several natural examples in the next section.

Associated to each family is a "symmetry type" which identifies the matrix ensemble that will be used to model the family. This correspondence is most easily seen in terms of the sign of the functional equation, which is analogous to the determinant of the matrix. If $A$ is unitary symplectic, then $\operatorname{det} A=1$ (i.e. $\phi=0$ ), and if $A$ is orthogonal, then $\operatorname{det} A= \pm 1$. Correspondingly, the functional equations for $L$-functions with unitary symmetry involve a (generally complex) phase factor, whereas for $L$-functions with symplectic symmetry this phase factor is unity, and in the case of orthogonal symmetry it is either +1 or -1 .

While the sign of the functional equation can sometimes suggest the symmetry type of the family, in general it requires a calculation to determine the symmetry type. One possible calculation is to determine the moments of the family near the critical point, as described in this paper. Comparison with the corresponding random matrix average can then be used to determine the symmetry type. Another possibility is to determine the density of the low-lying zeros of the family.

1.3. Example families and moments of $L$-functions. We now give examples of families of primitive $L$-functions and describe the associated mean values. The families we consider here are of a special form, which is described in Section 3. In preparation for the comparison with random matrices in the next section, we will classify the example families according to 
their symmetry type: Unitary, Orthogonal, and Symplectic. For the Orthogonal symmetry type we recognize three cases: $S O, \mathrm{O}^{-}$, and $O$, corresponding respectively to Orthogonal families in which the functional equation has $\varepsilon=1$, or $\varepsilon=-1$, or $\varepsilon= \pm 1$ equally often. Note that each family is a partially ordered set, and the order is determined by a quantity called the "conductor" of the $L$-function. The mean values given below are conjectural for all but a few small values of $k$. For a general discussion of these mean values and some more examples, see $[\mathrm{CF}$.

Unitary examples:

1) $\{L(s+i y) \mid y \geq 0\}$, ordered by $y$, where $L(s)$ is any primitive $L$-function. These are the only known continuous families of $L$-functions (Sarnak's rigidity conjecture).

2) $\{L(s, \chi) \mid q$ a positive integer, $\chi$ a primitive character $\bmod q\}$ ordered by $q$.

An example conjectured mean value for integer $k$ is:

$$
\int_{0}^{T}\left|\zeta\left(\frac{1}{2}+i t\right)\right|^{2 k} d t=T \mathcal{P}_{k}(\log T)+O\left(T^{\frac{1}{2}+\varepsilon}\right),
$$

for some polynomial $\mathcal{P}_{k}$ of degree $k^{2}$ with leading coefficient $g_{k} a_{k} / k^{2}$ !, where

$$
\begin{aligned}
a_{k} & =\prod_{p}\left(1-\frac{1}{p}\right)^{k^{2}} \sum_{m=0}^{\infty}\left(\frac{\Gamma(m+k)}{m ! \Gamma(k)}\right)^{2} p^{-m} \\
& =\prod_{p}\left(1-\frac{1}{p}\right)^{(k-1)^{2}} \sum_{j=0}^{k-1}\left(\begin{array}{c}
k-1 \\
j
\end{array}\right)^{2} p^{-j}
\end{aligned}
$$

and

$$
g_{k}=k^{2} ! \prod_{j=0}^{k-1} \frac{j !}{(k+j) !} .
$$

(The placement of $k^{2}$ ! is to ensure that $g_{k}$ is an integer [CF].) The above conjecture has been proven for $k=1$, 2. See [HL, II, A, Ko, H-B1, C], Mot]. When $k=2$ our conjectured error term of $O\left(T^{\frac{1}{2}+\varepsilon}\right)$ has only been obtained in the case of a smooth weight function [Iv1].

Symplectic examples:

3) $\left\{L\left(s, \chi_{d}\right) \mid d\right.$ a fundamental discriminant, $\left.\chi_{d}(n)=\left(\frac{d}{n}\right)\right\}$ ordered by $|d|$.

4) $\left\{L\left(s, \operatorname{sym}^{2} f\right) \mid f \in S_{k}\left(\Gamma_{0}(1)\right), k\right.$ a positive even integer $\}$, ordered by $k$.

An example conjectured mean value is:

$$
\sum_{|d| \leq D}^{*} L\left(\frac{1}{2}, \chi_{d}\right)^{k}=\frac{6}{\pi^{2}} D \mathcal{Q}_{k}(\log D)+O\left(D^{\frac{1}{2}+\varepsilon}\right)
$$

where $\sum^{*}$ is over fundamental discriminants, $\chi_{d}(n)=\left(\frac{d}{n}\right)$ is the Kronecker symbol, and the sum is over all real, primitive Dirichlet characters of conductor up to $D$. Here $\mathcal{Q}_{k}$ is a polynomial of degree $k(k+1) / 2$, with leading coefficient $g_{k} a_{k} /(k(k+1) / 2)$ !, where

$$
a_{k}=\prod_{p} \frac{\left(1-\frac{1}{p}\right)^{\frac{k(k+1)}{2}}}{1+\frac{1}{p}}\left(\frac{\left(1-\frac{1}{\sqrt{p}}\right)^{-k}+\left(1+\frac{1}{\sqrt{p}}\right)^{-k}}{2}+\frac{1}{p}\right)
$$


and

$$
g_{k}=(k(k+1) / 2) ! \prod_{j=1}^{k} \frac{j !}{(2 j) !} .
$$

The main term of this conjecture has been proven for $k=1,2,3$, and the case of $k=4$ is almost within reach of current methods. See GV, [J, So,

Orthogonal examples:

5) $\left\{L(s, f) \mid f \in S_{k}\left(\Gamma_{0}(N)\right), N\right.$ fixed, $k$ a positive even integer $\}$, ordered by $k$.

6) $\left\{L(s, f) \mid f \in S_{k}\left(\Gamma_{0}(N)\right)\right.$, $k$ fixed, $N$ a positive integer $\}$, ordered by $N$.

An example conjectured mean value is:

$$
\sum_{f \in H_{2}(q)} L_{f}\left(\frac{1}{2}, f\right)^{k}=\frac{q}{3} \mathcal{R}_{k}(\log q)+O\left(q^{\frac{1}{2}+\varepsilon}\right),
$$

where $\mathrm{H}_{2}(q)$ is the collection of Hecke newforms of weight 2 and squarefree level $q$. Here $\mathcal{R}_{k}$ is a polynomial of degree $k(k-1) / 2$, with leading coefficient $g_{k} a_{k} /(k(k-1) / 2)$ !, where

$$
a_{k}=\prod_{p \nmid q}\left(1-\frac{1}{p}\right)^{\frac{k(k-1)}{2}} \frac{2}{\pi} \int_{0}^{\pi} \sin ^{2} \theta\left(\frac{e^{i \theta}\left(1-\frac{e^{i \theta}}{\sqrt{p}}\right)^{-1}-e^{-i \theta}\left(1-\frac{e^{-i \theta}}{\sqrt{p}}\right)^{-1}}{e^{i \theta}-e^{-i \theta}}\right)^{k} d \theta
$$

and

$$
g_{k}=2^{k-1}(k(k-1) / 2) ! \prod_{j=1}^{k-1} \frac{j !}{(2 j) !} .
$$

The main term of this conjecture has been proven for $k=1,2,3,4$, in the case that $q$ is prime. See [D, DFI, KMV]. Also see Ivić [Iv2 for the analogous mean values for Maass forms.

The above examples are merely meant to give a flavor of the types of families which are of current interest.

The above cases, and their random matrix analogues, have been extensively discussed from the perspective of the leading terms in the asymptotic expansions. See CF, KS1, KS2. In the present paper we extend that work to include all of the terms in the above mean values (ie., all coefficients in the conjectured polynomials), which we recover from a more general mean value involving a product of $L$-functions whose arguments are free parameters. In the next two sections we describe these more general mean values, discuss their random matrix analogues, and then state our results and conjectures.

1.4. Shifted moments. A key point in this paper is that the structure of mean values of $L$-functions is more clearly revealed if one considers the average of a product of $L$-functions, where each $L$-function is evaluated at a location slightly shifted from the critical point. The example mean values given in the previous section can be obtained by allowing the shifts to tend to zero. 
Let $\alpha=\left(\alpha_{1}, \ldots, \alpha_{2 k}\right)$, where throughout the paper we assume $\left|\Re \alpha_{j}\right|<\frac{1}{2}$, and suppose that $g(t)$ is a suitable weight function. The mean values we consider are

$$
I_{k}(L, \alpha, g)=\int_{-\infty}^{\infty} Z_{L}\left(\frac{1}{2}+\alpha_{1}+i t\right) \cdots Z_{L}\left(\frac{1}{2}+\alpha_{2 k}+i t\right) g(t) d t
$$

and, with $\alpha=\left(\alpha_{1}, \ldots, \alpha_{k}\right)$,

$$
S_{k}(\mathcal{F}, \alpha, g)=\sum_{L \in \mathcal{F}} Z_{L}\left(\frac{1}{2}+\alpha_{1}\right) \cdots Z_{L}\left(\frac{1}{2}+\alpha_{k}\right) g(c(L))
$$

In the first case it is assumed that $L(s)$ is a primitive $L$-function, and in the second $\mathcal{F}$ is family of primitive $L$-functions partially ordered by $\log$ conductor $c(L)$.

We refer to $g$ as a "suitable" weight function, but we leave that term undefined. An example of a suitable weight function is $g(x)=f(x / T)$, where $f$ is real, nonnegative, bounded, and integrable on the positive real line.

The random matrix analogs of the above expressions are

$$
J_{k}(U(N), \alpha)=\int_{U(N)} \mathcal{Z}_{A}\left(e^{-\alpha_{1}}\right) \cdots \mathcal{Z}_{A}\left(e^{-\alpha_{2 k}}\right) d A,
$$

where $\alpha=\left(\alpha_{1}, \ldots, \alpha_{2 k}\right)$ and the average is over Haar measure on $U(N)$, and

$$
J_{k}(G(N), \alpha)=\int_{G(N)} \mathcal{Z}_{A}\left(e^{-\alpha_{1}}\right) \cdots \mathcal{Z}_{A}\left(e^{-\alpha_{k}}\right) d A
$$

where $G(N)$ is $U S p(2 N), O^{-}(2 N)$, or $S O(2 N)$ and $\alpha=\left(\alpha_{1}, \ldots, \alpha_{k}\right) . O^{-}(2 N)$ is defined as the collection of orthogonal $2 N \times 2 N$ matrices with determinant negative one. Haar measure on $U S p(2 N)$ and $O(2 N)$ determines the weighting for the averages.

In the next section we compare our conjectures for the $L$-function mean values with exact formulae for the random matrix averages.

1.5. Main results and example conjectures. We state our main results and conjectures here. We give example conjectures for the full main term in shifted mean values of number theoretic interest; these examples illustrate our methods and cover the three symmetry types of families of $L$-functions. We also give a corresponding theorem about the random matrix analogue of these mean values for each of the three compact matrix ensembles.

We present our results in pairs: a conjecture for an $L$-function mean value, followed by a theorem, quoted from CFKRS, for the corresponding average of characteristic polynomials. For each pair the parts of each formula match according to the scheme described in Section 1.2. In particular, the scaling of the large parameter is determined by equating log conductors. In the random matrix formula the integrand contains a term $\left(1-e^{ \pm z_{m}-z_{\ell}}\right)^{-1}$, which has a simple pole at $z_{\ell}= \pm z_{m}$. In the $L$-function formula this corresponds to the term containing all of the arithmetic information, which is of the form $\zeta\left(1+z_{i} \mp z_{j}\right)$ times an Euler product, and so also has a simple pole at $z_{i}= \pm z_{j}$.

The formulae are written in terms of contour integrals and involve the Vandermonde:

$$
\Delta\left(z_{1}, \ldots, z_{m}\right)=\prod_{1 \leq i<j \leq m}\left(z_{j}-z_{i}\right) .
$$

We also set $e(z)=e^{2 \pi i z}$. 
Conjecture 1.5.1. Suppose $g(t)$ is a suitable weight function. Then

$$
\int_{-\infty}^{\infty}\left|\zeta\left(\frac{1}{2}+i t\right)\right|^{2 k} g(t) d t=\int_{-\infty}^{\infty} P_{k}\left(\log \frac{t}{2 \pi}\right)\left(1+O\left(t^{-\frac{1}{2}+\varepsilon}\right)\right) g(t) d t
$$

where $P_{k}$ is the polynomial of degree $k^{2}$ given by the $2 k$-fold residue

$$
P_{k}(x)=\frac{(-1)^{k}}{k !^{2}} \frac{1}{(2 \pi i)^{2 k}} \oint \cdots \oint \frac{G\left(z_{1}, \ldots, z_{2 k}\right) \Delta^{2}\left(z_{1}, \ldots, z_{2 k}\right)}{\prod_{j=1}^{2 k} z_{j}^{2 k}} e^{\frac{x}{2} \sum_{j=1}^{k} z_{j}-z_{k+j}} d z_{1} \ldots d z_{2 k},
$$

where one integrates over small circles about $z_{i}=0$, with

$$
G\left(z_{1}, \ldots, z_{2 k}\right)=A_{k}\left(z_{1}, \ldots, z_{2 k}\right) \prod_{i=1}^{k} \prod_{j=1}^{k} \zeta\left(1+z_{i}-z_{k+j}\right),
$$

and $A_{k}$ is the Euler product

$$
A_{k}(z)=\prod_{p} \prod_{i=1}^{k} \prod_{j=1}^{k}\left(1-\frac{1}{p^{1+z_{i}-z_{k+j}}}\right) \int_{0}^{1} \prod_{j=1}^{k}\left(1-\frac{e(\theta)}{p^{\frac{1}{2}+z_{j}}}\right)^{-1}\left(1-\frac{e(-\theta)}{p^{\frac{1}{2}-z_{k+j}}}\right)^{-1} d \theta .
$$

More generally,

$$
I_{k}(\zeta, \alpha, g)=\int_{-\infty}^{\infty} P_{k}\left(\log \frac{t}{2 \pi}, \alpha\right)\left(1+O\left(t^{-\frac{1}{2}+\varepsilon}\right)\right) g(t) d t
$$

where

$$
P_{k}(x, \alpha)=\frac{(-1)^{k}}{k !^{2}} \frac{1}{(2 \pi i)^{2 k}} \oint \cdots \oint \frac{G\left(z_{1}, \ldots, z_{2 k}\right) \Delta^{2}\left(z_{1}, \ldots, z_{2 k}\right)}{\prod_{j=1}^{2 k} \prod_{i=1}^{2 k}\left(z_{j}-\alpha_{i}\right)} e^{\frac{x}{2} \sum_{j=1}^{k} z_{j}-z_{k+j}} d z_{1} \ldots d z_{2 k},
$$

with the path of integration being small circles surrounding the poles $\alpha_{i}$.

A general version of the above conjecture is given in Conjecture 2.5.4.

Theorem 1.5.2. In the notation of Section 1.4 we have

$$
J_{k}(U(N), 0)=\prod_{j=0}^{k-1}\left(\frac{j !}{(k+j) !} \prod_{i=1}^{k}(N+i+j)\right) .
$$

More generally, with

$$
G\left(z_{1}, \ldots, z_{2 k}\right)=\prod_{i=1}^{k} \prod_{j=1}^{k}\left(1-e^{-z_{i}+z_{j+k}}\right)^{-1}
$$

we have

$$
J_{k}(U(N), \alpha)=\frac{(-1)^{k}}{k !^{2}} \frac{1}{(2 \pi i)^{2 k}} \oint \cdots \oint \frac{G\left(z_{1}, \ldots, z_{2 k}\right) \Delta^{2}\left(z_{1}, \ldots, z_{2 k}\right)}{\prod_{i=1}^{2 k} \prod_{j=1}^{2 k}\left(z_{j}-\alpha_{i}\right)} e^{\frac{N}{2} \sum_{j=1}^{k} z_{j}-z_{k+j}} d z_{1} \cdots d z_{2 k} .
$$


Comments on the formulae:

(1) Let $\alpha_{i} \rightarrow 0$ in the second part of the Conjecture to obtain the first part of the Conjecture.

(2) The structures of $J_{k}(U(N), \alpha)$ and $P_{k}(x, \alpha)$ are identical in that the functions $G\left(z_{1}, \ldots, z_{2 k}\right)$ have simple poles at $z_{i}=z_{k+j}$.

(3) The local factors of $A_{k}(\alpha)$ are polynomials in $p^{-1}$ and $p^{-\alpha_{i}}, i=1, \ldots, k$, as seen from Theorem 2.6.2. Since $A_{k}(\alpha)$ comes from a symmetric expression, it is also a polynomial in $p^{\alpha_{i+k}}, i=1, \ldots, k$. This is discussed in Section 2.6. Note also that $a_{k}$ in (1.3.2) equals $A_{k}(0, \ldots, 0)$, as shown in Section 2.7

(4) That $P_{k}(x)$ is actually a polynomial of degree $k^{2}$ can be seen by considering the order of the pole at $z_{j}=0$. We wish to extract from the numerator of the integrand, the coefficient of $\prod z_{i}^{2 k-1}$, a polynomial of degree $2 k(2 k-1)$. The Vandermonde determinant squared is a homogeneous polynomial of degree $2 k(2 k-1)$. However, the poles coming from the $\zeta\left(1+z_{i}-z_{k+j}\right)$ cancel $k^{2}$ of the Vandermonde factors. This requires us, in computing the residue, to take, in the Taylor expansion of $\exp \left(\frac{x}{2} \sum_{1}^{k} z_{j}-z_{k+j}\right)$, terms up to degree $k^{2}$.

(5) The fact that $P_{k}\left(\log \frac{t}{2 \pi}\right)$ is a polynomial in $\log \frac{t}{2 \pi}$ of degree $k^{2}$ corresponds nicely to the formula for $J_{k}(U(N), 0)$, which is a polynomial of degree $k^{2}$ in $N$. Equating the density of the Riemann zeros at height $t$ with the density of the random matrix eigenvalues suggests the familiar equivalence $N=\log \frac{t}{2 \pi}$. In this paper we view this as equating conductors.

(6) The leading term of $P_{k}(x)$ coincides with the leading term conjectured by Keating and Snaith (see Section [2.7). The full polynomial $P_{k}(x)$ agrees, when $k=1$ and $k=2$, with known theorems (see Sections [1.6 and 1.7).

(7) We can recover the polynomial $\mathcal{P}_{k}$ in (1.3.1) from $P_{k}$ by taking $g(t)=\chi_{[0, T]}(t)$ in the conjecture.

(8) The multiple integrals in the Theorem and the Conjecture can be written as combinatorial sums. See Section 2 where a detailed derivation of our conjecture is given.

(9) Our conjecture concerning the order of the error term is based on our numerical calculations (see Section 5) and examination of examples in the literature.

Conjecture 1.5.3. Suppose $g(u)$ is a suitable weight function with support in either $(0, \infty)$ or $(-\infty, 0)$, and let $X_{d}(s)=|d|^{\frac{1}{2}-s} X(s, a)$ where $a=0$ if $d>0$ and $a=1$ if $d<0$, and

$$
X(s, a)=\pi^{s-\frac{1}{2}} \frac{\Gamma\left(\frac{1+a-s}{2}\right)}{\Gamma\left(\frac{s+a}{2}\right)} .
$$

That is, $\chi_{d}(s)$ is the factor in the functional equation $L\left(s, \chi_{d}\right)=\varepsilon_{d} X_{d}(s) L\left(1-s, \chi_{d}\right)$. Summing over fundamental discriminants $d$ we have

$$
\sum_{d}^{*} L\left(\frac{1}{2}, \chi_{d}\right)^{k} g(|d|)=\sum_{d}^{*} Q_{k}(\log |d|)\left(1+O\left(|d|^{-\frac{1}{2}+\varepsilon}\right)\right) g(|d|)
$$


where $Q_{k}$ is the polynomial of degree $k(k+1) / 2$ given by the $k$-fold residue (1.5.12)

$$
Q_{k}(x)=\frac{(-1)^{k(k-1) / 2} 2^{k}}{k !} \frac{1}{(2 \pi i)^{k}} \oint \cdots \oint \frac{G\left(z_{1}, \ldots, z_{k}\right) \Delta\left(z_{1}^{2}, \ldots, z_{k}^{2}\right)^{2}}{\prod_{j=1}^{k} z_{j}^{2 k-1}} e^{\frac{x}{2} \sum_{j=1}^{k} z_{j}} d z_{1} \ldots d z_{k},
$$

where

$$
G\left(z_{1}, \ldots, z_{k}\right)=A_{k}\left(z_{1}, \ldots, z_{k}\right) \prod_{j=1}^{k} X\left(\frac{1}{2}+z_{j}, a\right)^{-\frac{1}{2}} \prod_{1 \leq i \leq j \leq k} \zeta\left(1+z_{i}+z_{j}\right),
$$

and $A_{k}$ is the Euler product, absolutely convergent for $\left|\Re z_{j}\right|<\frac{1}{2}$, defined by

$$
\begin{aligned}
A_{k}\left(z_{1}, \ldots, z_{k}\right)= & \prod_{p} \prod_{1 \leq i \leq j \leq k}\left(1-\frac{1}{p^{1+z_{i}+z_{j}}}\right) \\
& \times\left(\frac{1}{2}\left(\prod_{j=1}^{k}\left(1-\frac{1}{p^{\frac{1}{2}+z_{j}}}\right)^{-1}+\prod_{j=1}^{k}\left(1+\frac{1}{p^{\frac{1}{2}+z_{j}}}\right)^{-1}\right)+\frac{1}{p}\right)\left(1+\frac{1}{p}\right)^{-1} .
\end{aligned}
$$

More generally, if $\mathcal{F}$ is the family of real primitive Dirichlet $L$-functions then

$$
S_{k}(\mathcal{F}, \alpha, g)=\sum_{d}^{*} Q_{k}(\log |d|, \alpha)\left(1+O\left(|d|^{-\frac{1}{2}+\varepsilon}\right)\right) g(|d|),
$$

in which

$$
\begin{aligned}
Q_{k}(x, \alpha)= & \frac{(-1)^{k(k-1) / 2} 2^{k}}{k !} \frac{1}{(2 \pi i)^{k}} \\
& \quad \times \oint \cdots \oint \frac{G\left(z_{1}, \ldots, z_{k}\right) \Delta\left(z_{1}^{2}, \ldots, z_{k}^{2}\right)^{2} \prod_{j=1}^{k} z_{j}}{\prod_{i=1}^{k} \prod_{j=1}^{k}\left(z_{j}-\alpha_{i}\right)\left(z_{j}+\alpha_{i}\right)}
\end{aligned}
$$

where the path of integration encloses the $\pm \alpha$ 's.

Theorem 1.5.4. In the notation of Section 1.4 we have

$$
J_{k}(U S p(2 N), 0)=\left(2^{k(k+1) / 2} \prod_{j=1}^{k} \frac{j !}{(2 j) !}\right) \prod_{1 \leq i \leq j \leq k}\left(N+\frac{i+j}{2}\right) .
$$

More generally, with

$$
G\left(z_{1}, \ldots, z_{k}\right)=\prod_{1 \leq i \leq j \leq k}\left(1-e^{-z_{i}-z_{j}}\right)^{-1}
$$

we have

$$
\begin{aligned}
J_{k}(U S p(2 N), \alpha)= & \frac{(-1)^{k(k-1) / 2} 2^{k}}{k !} \frac{1}{(2 \pi i)^{k}} \\
& \quad \times \oint \cdots \oint \frac{G\left(z_{1}, \ldots, z_{k}\right) \Delta\left(z_{1}^{2}, \ldots, z_{k}^{2}\right)^{2} \prod_{j=1}^{k} z_{j}}{\prod_{i=1}^{k} \prod_{j=1}^{k}\left(z_{j}-\alpha_{i}\right)\left(z_{j}+\alpha_{i}\right)}
\end{aligned}
$$


where the contours of integration enclose the $\pm \alpha$ 's.

Comments:

(1) When comparing Theorem 1.5.4 with Conjecture 1.5.3, equating log conductors (i.e., the density of zeros) gives the equivalence

$$
2 N=\operatorname{cond}(d):=\log (|d| / \pi)+\left(\Gamma^{\prime} / \Gamma\right)\left(\frac{1}{4}+a\right) .
$$

The conductor we use here should be contrasted with the "usual" conductor associated with Dirichlet $L$-functions: $\log (|d| / \pi)-\log (2)$. We believe this difference is significant, so we discuss it briefly.

The following manipulations show that our conductor arises naturaly. In the derivation of the conjecture, one encounters the function $X_{d}\left(\frac{1}{2}+z\right)^{-\frac{1}{2}}$, which can we rewritten in several ways:

$$
\begin{aligned}
X_{d}\left(\frac{1}{2}+z\right)^{-\frac{1}{2}} & =e^{\frac{1}{2} \log d \cdot z} X\left(\frac{1}{2}+z, a\right)^{-\frac{1}{2}} \\
& =e^{\frac{1}{2} \operatorname{cond}(d) \cdot z} \mathcal{G}(z)
\end{aligned}
$$

where $\mathcal{G}(z)=1+O\left(z^{3}\right)$. In the statement of the conjecture we used the first line of (1.5.20), incorporating the product over $X\left(\frac{1}{2}+z, a\right)^{-\frac{1}{2}}$ into the factor $G\left(z_{1}, \ldots, z_{k}\right)$. If we chose instead to use the second line of (1.5.20), then the conjecture would be written as a sum over $Q_{k}(\operatorname{cond}(d))$. One would still have that $Q_{k}$ is a polynomial of degree $k(k+1) / 2$. Since $\mathcal{G}(z)=1+O\left(z^{3}\right)$, the first 3 leading terms in that polynomial would not explicitly depend on the factor $X_{d}$ from the functional equation, although the lower degree terms would. This phenomenon does not occur for moments of $L$-functions in $t$-aspect.

(2) $\mathcal{Q}_{k}$ in (1.3.4) can be recovered from $Q_{k}$ above by taking $g(|d|)=\chi_{[0, D]}(|d|)$, and using the estimate $\sum_{-D<d<0}^{*} 1=3 D / \pi^{2}+O\left(D^{\frac{1}{2}+\varepsilon}\right)$; the same estimate holds for positive $d$.

(3) A heuristic derivation of Conjecture 1.5.3 is given in Section 4.4.

(4) The leading term of $Q_{k}$ coincides with the leading term conjectured by Keating and Snaith KS2]. The calculation is analogous to the one given in Section 2.7

Conjecture 1.5.5. Suppose $q$ is squarefree, let $H_{n}(q)$ be the set of newforms in $S_{n}\left(\Gamma_{0}(q)\right)$, and let

$$
X_{n, q}(s)=\left(\frac{q}{4 \pi^{2}}\right)^{\frac{1}{2}-s} \frac{\Gamma\left(\frac{1}{2}-s+\frac{n}{2}\right)}{\Gamma\left(s-\frac{1}{2}+\frac{n}{2}\right)}
$$

be the factor in the functional equation $L_{f}(s)=\varepsilon_{n, q} X_{n, q}(s) L_{f}(1-s)$ for the L-functions associated to $f \in H_{n}(q)$. Then

$$
\sum_{f \in H_{n}(q)} L_{f}\left(\frac{1}{2}\right)^{k}\langle f, f\rangle^{-1}=\sum_{f \in H_{n}(q)} R_{k}(n, q)\langle f, f\rangle^{-1}\left(1+O(n q)^{-\frac{1}{2}+\varepsilon}\right)
$$

as $n q \rightarrow \infty$, where $R_{k}(n, q)$ is given by the $k$-fold residue

$$
R_{k}=\frac{(-1)^{k(k-1) / 2} 2^{k-1}}{k !} \frac{1}{(2 \pi i)^{k}} \oint \ldots \oint \frac{G\left(z_{1}, \ldots, z_{k}\right) \Delta\left(z_{1}^{2}, \ldots, z_{k}^{2}\right)^{2}}{\prod_{j=1}^{k} z_{j}^{2 k-1}} \prod_{j=1}^{k} X_{n, q}\left(\frac{1}{2}+z_{j}\right)^{-\frac{1}{2}} d z_{1} \ldots d z_{k},
$$


where

$$
G\left(z_{1}, \ldots, z_{k}\right)=A_{k}\left(z_{1}, \ldots, z_{k}\right) \prod_{1 \leq i<j \leq k} \zeta\left(1+z_{i}+z_{j}\right)
$$

and $A_{k}$ is the Euler product which is absolutely convergent for $\left|\Re z_{j}\right|<\frac{1}{2}$, with $j=1, \ldots, k$, defined by

$$
A_{k}\left(z_{1}, \ldots, z_{k}\right)=\prod_{p \nmid q} \prod_{1 \leq i<j \leq k}\left(1-\frac{1}{p^{1+z_{i}+z_{j}}}\right)
$$

$$
\times \frac{2}{\pi} \int_{0}^{\pi} \sin ^{2} \theta \prod_{j=1}^{k} \frac{e^{i \theta}\left(1-\frac{e^{i \theta}}{p^{\frac{1}{2}+z_{j}}}\right)^{-1}-e^{-i \theta}\left(1-\frac{e^{-i \theta}}{p^{\frac{1}{2}+z_{j}}}\right)^{-1}}{e^{i \theta}-e^{-i \theta}} d \theta .
$$

To state the more general version of Conjecture 1.5.5, involving a sum of products of $L_{f}\left(\frac{1}{2}+u_{j}\right)$, it is natural also to consider the sums over even $f$ and odd $f$ separately. See Conjectures 4.5.1 and 4.5.2.

Theorem 1.5.6. In the notation of Section 1.4 we have

$$
J_{k}(S O(2 N), 0)=\left(2^{k(k+1) / 2} \prod_{j=1}^{k-1} \frac{j !}{(2 j) !}\right) \prod_{0 \leq i<j \leq k-1}\left(N+\frac{i+j}{2}\right) .
$$

More generally, with

$$
G\left(z_{1}, \ldots, z_{k}\right)=\prod_{1 \leq \ell<m \leq k}\left(1-e^{-z_{m}-z_{\ell}}\right)^{-1}
$$

we have

$$
\begin{aligned}
J_{k}(S O(2 N), \alpha)= & \frac{(-1)^{k(k-1) / 2} 2^{k}}{k !} \frac{1}{(2 \pi i)^{k}} \\
& \quad \times \oint \cdots \oint \frac{G\left(z_{1}, \ldots, z_{k}\right) \Delta\left(z_{1}^{2}, \ldots, z_{k}^{2}\right)^{2} \prod_{j=1}^{k} z_{j}}{\prod^{k} \prod^{N} \prod_{j=1}^{k}\left(z_{j}-\alpha_{i}\right)\left(z_{j}+\alpha_{i}\right)} d z_{1} \cdots d z_{k}
\end{aligned}
$$

and

$$
\begin{aligned}
J_{k}\left(O^{-}(2 N), \alpha\right)= & i^{-k} \frac{(-1)^{k(k-1) / 2} 2^{k}}{k !} \frac{1}{(2 \pi i)^{k}} \\
& \times \oint \cdots \oint \frac{G\left(z_{1}, \ldots, z_{k}\right) \Delta\left(z_{1}^{2}, \ldots, z_{k}^{2}\right)^{2} \prod_{j=1}^{k} \alpha_{j}}{\prod_{i=1}^{k} \prod_{j=1}^{k}\left(z_{j}-\alpha_{i}\right)\left(z_{j}+\alpha_{i}\right)}
\end{aligned}
$$

Comments:

(1) $R_{k}(n, q)$ does not actually depend on $f \in H_{n}(q)$. We write (1.5.22) in this manner to stress that $R_{k}(n, q)$ is the expected value of $L_{f}\left(\frac{1}{2}\right)^{k}$. 
(2) To compare Theorem 1.5.6 and Conjecture 1.5.5, equating conductors gives the equivalence

$$
\begin{aligned}
2 N & =\operatorname{cond}(n, q):=\log \left(q / 4 \pi^{2}\right)+\left(\Gamma^{\prime} / \Gamma\right)(n / 2) \\
& =\log \left(q n / 8 \pi^{2}\right)+O\left(n^{-1}\right) .
\end{aligned}
$$

One can express the conjectured mean value in terms of the conductor in the following way. In (1.5.23) we can write

$$
X_{n, q}\left(\frac{1}{2}+z_{j}\right)^{-\frac{1}{2}}=e^{\frac{1}{2} \operatorname{cond}(n, q) \cdot z_{j}} \mathcal{G}\left(z_{j}\right),
$$

where $\mathcal{G}\left(z_{j}\right)=1+O\left(z^{3}\right)$. As in Conjecture 1.5.3. we can express $R_{k}(n, q)$ as a polynomial in the conductor, the first 3 terms of which do not depend on the $X_{n, q}$ factor in the functional equation.

(3) All of our conjectures naturally contain a factor of the form $\prod X\left(\frac{1}{2} \pm z_{j}\right)^{-\frac{1}{2}}$, it just happens that in some cases $X\left(\frac{1}{2} \pm z_{j}\right)$ can be closely approximated by a simple function of the conductor. It is interesting that this same factor occurs in all of the random matrix moments. In that case $X(s)=s^{-M}$, where $M=N$ or $2 N$, so in the formula for the moments there occurs $\prod X\left(e^{ \pm z_{j}}\right)^{-\frac{1}{2}}=e^{\frac{M}{2} \sum \pm z_{j}}$.

1.6. 2nd Moment of the Riemann zeta-function. Now we consider the 2 nd moment of the Riemann zeta-function in detail, putting our results in the context of the literature.

Ingham's result [I] on the 2nd moment can be stated as

$$
\int_{0}^{T} \zeta(s+\alpha) \zeta(1-s-\beta) d t=\int_{0}^{T}\left(\zeta(1+\alpha-\beta)+\tau^{\beta-\alpha} \zeta(1+\beta-\alpha)\right)\left(1+O\left(t^{-\frac{1}{2}+\varepsilon}\right)\right) d t
$$

where $s=\frac{1}{2}+i t$ and $\tau=\tau(t)=\frac{|t|}{2 \pi}$; this is valid for $|\alpha|,|\beta|<\frac{1}{2}$. If we let $\alpha$ and $\beta$ approach 0 here we obtain Ingham's theorem

$$
\int_{0}^{T}\left|\zeta\left(\frac{1}{2}+i t\right)\right|^{2} d t=\int_{0}^{T}\left(\log \frac{t}{2 \pi}+2 \gamma\right) d t+O\left(T^{\frac{1}{2}+\varepsilon}\right)
$$

Our conjecture is compatible with these results, because, when $k=1$, the function $G\left(\alpha_{1}, \alpha_{2}\right)$ that appears in Conjecture 1.5.1 equals $\zeta\left(1+\alpha_{1}-\alpha_{2}\right)$. Computing the residue, we find

$$
\begin{aligned}
P_{1}(x) & =\frac{1}{4 \pi^{2}} \oint \oint \frac{\zeta\left(1+z_{1}-z_{2}\right)\left(z_{2}-z_{1}\right)^{2}}{z_{1}^{2} z_{2}^{2}} e^{\frac{x}{2}\left(z_{1}-z_{2}\right)} d z_{1} d z_{2} \\
& =x+2 \gamma .
\end{aligned}
$$

The second moment with a different weighting is now given; this theorem is a slight variation of the theorem of Kober presented in Titchmarsh [T] and was inspired by the numerical calculations described in Section 5.1 .

Theorem 1.6.1. Let

$$
I(\alpha, \beta, \delta)=\int_{0}^{\infty} \zeta\left(\frac{1}{2}+i t+\alpha\right) \zeta\left(\frac{1}{2}-i t-\beta\right) e^{-\delta t} d t .
$$

Then, for any $\eta>0,|\alpha|,|\beta| \leq \frac{1}{2}-\eta$ and $|\arg \delta| \leq \frac{\pi}{2}-\eta$, we have

$$
I(\alpha, \beta, \delta)=\int_{0}^{\infty}\left(\zeta(1+\alpha-\beta)+\left(\frac{t}{2 \pi}\right)^{\alpha-\beta} \zeta(1-\alpha+\beta)\right) e^{-\delta t} d t+C_{\delta}(\alpha, \beta)+O(\delta \log 1 / \delta)
$$


uniformly in $\alpha$, $\beta$, and $\delta$ where $C_{\delta}(\alpha, \beta) \ll \log 1 / \delta$ uniformly in $\alpha$ and $\beta$ and where $C_{\delta}(\alpha,-\alpha)=-2 \pi \zeta(2 \alpha)$.

We restate the case $\beta=-\alpha$ as

Corollary 1.6.2. For any fixed $\alpha$ with $|\alpha|<\frac{1}{2}$, we have

$$
\begin{array}{ll}
\lim _{\delta \rightarrow 0}\left(\int_{0}^{\infty}\left|\zeta\left(\frac{1}{2}+\alpha+i t\right)\right|^{2} e^{-\delta t} d t-\right. & \left.\int_{0}^{\infty}\left(\zeta(1+2 \alpha)+\left(\frac{t}{2 \pi}\right)^{2 \alpha} \zeta(1-2 \alpha)\right) e^{-\delta t} d t\right) \\
& =-\pi \zeta(2 \alpha)-(2 \pi)^{2 \alpha} \zeta(1-2 \alpha) \Gamma(1-2 \alpha) \sin \pi \alpha \\
& =-2 \pi \zeta(2 \alpha) .
\end{array}
$$

Note that

$$
\lim _{x \rightarrow 0}\left(\zeta(1+x)+w^{-x} \Gamma(1-x) \zeta(1-x)\right)=(2 \gamma+\log w) .
$$

Thus, letting $\alpha \rightarrow 0$, gives

\section{Corollary 1.6.3.}

$$
\lim _{\delta \rightarrow 0}\left(\int_{0}^{\infty}\left|\zeta\left(\frac{1}{2}+i t\right)\right|^{2} e^{-\delta t} d t-\int_{0}^{\infty}\left(2 \gamma+\log \left(\frac{t}{2 \pi}\right)\right) e^{-\delta t} d t\right)=\pi
$$

Remark: We discovered this Corollary after seeing the numerical results of Section 5.1 This result also follows from a result of Hafner and Ivić [HI].

1.7. 4th Moment of the Riemann zeta-function. Now we consider the 4th moment of the Riemann zeta-function in detail. Our discussion here builds upon work of Atkinson $\mathrm{A}$, Heath-Brown[H-B1], Conrey [C], and Motohashi Mot].

Examining Motohashi's results in detail, consider

$$
\int_{-\infty}^{\infty} \zeta\left(s+u_{1}\right) \ldots \zeta\left(s+u_{k}\right) \zeta\left(1-s+v_{1}\right) \ldots \zeta\left(1-s+v_{k}\right) g(t) d t
$$

for a function $g(t)$ which is analytic in a horizontal strip $|\Im(t)|<c$ and decays sufficiently rapidly. Motohashi obtains an exact formula for these moments for $k=1$ and $k=2$. We reformulate Motohashi's theorem $(k=2)$ in our context. Let

$$
C(v)=\frac{(2 \pi)^{v}}{2 \cos \frac{\pi}{2} v}
$$

and let

$$
G_{s}(u, v)=\frac{\Gamma(s-u)}{\Gamma(s-v)}
$$

Then, in a notation analogous to Motohashi's, the $k=2$ case of (1.7.1) equals

$$
L_{r}+L_{d}+L_{c}+L_{h}
$$

where $L_{r}$ is the (residual) main term which we are interested in here:

$$
L_{r}(u, v)=\int_{-\infty}^{\infty} W(t, u, v) g(t) d t
$$


with

$$
\begin{aligned}
W(t, u, v)= & C(0)\left(G_{s}(0,0)+G_{1-s}(0,0)\right) Z\left(u_{1}, u_{2}, v_{1}, v_{2}\right) \\
+ & C\left(u_{1}+v_{1}\right)\left(G_{s}\left(u_{1}, v_{1}\right)+G_{1-s}\left(u_{1}, v_{1}\right)\right) Z\left(-v_{1}, u_{2},-u_{1}, v_{2}\right) \\
& +C\left(u_{1}+v_{2}\right)\left(G_{s}\left(u_{1}, v_{2}\right)+G_{1-s}\left(u_{1}, v_{2}\right)\right) Z\left(-v_{2}, u_{2}, v_{1},-u_{1}\right) \\
+ & C\left(u_{2}+v_{1}\right)\left(G_{s}\left(u_{2}, v_{1}\right)+G_{1-s}\left(u_{2}, v_{1}\right)\right) Z\left(u_{1},-v_{1},-u_{2}, v_{2}\right) \\
+ & C\left(u_{2}+v_{2}\right)\left(G_{s}\left(u_{2}, v_{2}\right)+G_{1-s}\left(u_{2}, v_{2}\right)\right) Z\left(u_{1},-v_{2}, v_{1},-u_{2}\right) \\
+ & C\left(u_{1}+u_{2}+v_{1}+v_{2}\right)\left(G_{s}\left(u_{1}, v_{1}\right) G_{s}\left(u_{2}, v_{2}\right)+G_{1-s}\left(u_{1}, v_{1}\right) G_{1-s}\left(u_{2}, v_{2}\right)\right) \\
& \times Z\left(-v_{1},-v_{2},-u_{1},-u_{2}\right),
\end{aligned}
$$

where $s=\frac{1}{2}+i t$, and

$$
Z\left(u_{1}, u_{2}, v_{1}, v_{2}\right)=\frac{\zeta\left(1+u_{1}+v_{1}\right) \zeta\left(1+u_{1}+v_{2}\right) \zeta\left(1+u_{2}+v_{1}\right) \zeta\left(1+u_{2}+v_{2}\right)}{\zeta\left(2+u_{1}+u_{2}+v_{2}+v_{2}\right)} .
$$

This formula may be obtained from Motohashi's work (Mot] pp. 174 - 178) by a careful analysis of his terms together with appropriate use of the functional equation in the form

$$
\Gamma(s) \zeta(s)=\frac{(2 \pi)^{s}}{2 \cos \pi s / 2} \zeta(1-s)
$$

and some trigonometric identities.

If we use the approximation

$$
\frac{\Gamma(s+\alpha)}{\Gamma(s+\beta)}=(i|s|)^{\alpha-\beta}(1+O(1 /|s|),
$$

we have, using $\tau=|t| /(2 \pi)$,

$$
C(u)\left(G_{s}(u)+G_{1-s}(u)\right)=\tau^{-u}(1+O(1 / \tau))
$$

and

$$
C(u+v)\left(G_{s}(u) G_{s}(v)+G_{1-s}(u) G_{1-s}(v)\right)=\tau^{-u-v}(1+O(1 / \tau)) .
$$

We then have

$$
\begin{aligned}
W(t, u, v)= & \left(Z\left(u_{1}, u_{2}, v_{1}, v_{2}\right)+\tau^{-u_{1}-v_{1}} Z\left(-v_{1}, u_{2},-u_{1}, v_{2}\right)+\tau^{-u_{1}-v_{2}} Z\left(-v_{2}, u_{2}, v_{1},-u_{1}\right)\right. \\
& +\tau^{-u_{2}-v_{1}} Z\left(u_{1},-v_{1},-u_{2}, v_{2}\right)+\tau^{-u_{2}-v_{2}} Z\left(u_{1},-v_{2}, v_{1},-u_{2}\right) \\
& \left.+\tau^{-u_{1}-u_{2}-v_{1}-v_{2}} Z\left(-v_{1},-v_{2},-u_{1},-u_{2}\right)\right)(1+O(1 / \tau)) .
\end{aligned}
$$

By the formulae in Sections 2.2 and 2.5, the above agrees with the $k=2$ case of Conjecture 1.5.1.

A residue computation shows that our conjecture can be restated as

$$
\begin{aligned}
\int_{0}^{T}\left|\zeta\left(\frac{1}{2}+i t\right)\right|^{4} d t= & \int_{0}^{T} \frac{1}{2} \frac{1}{(2 \pi i)^{2}} \oint \oint\left(\frac{t}{2 \pi}\right)^{x+y} \\
& \times \frac{\zeta(1+x)^{4} \zeta(1+y)^{4}}{\zeta(1+x-y) \zeta(1+y-x) \zeta(2+2 x+2 y)} d x d y d t+O\left(T^{\frac{1}{2}+\varepsilon}\right),
\end{aligned}
$$

where we integrate around small circles centered on the origin. This is in contrast to Conjecture 1.5.1, which when $k=2$ expresses the formula in terms of four contour integrals. It 
may be that our formulae can be similarly simplified for all $k$, but we have not succeeded in doing so.

\section{Moments in $t$-ASPECT}

The principle behind our method of conjecturing mean values is that the Dirichlet series coefficients of $L$-functions have an approximate orthogonality relation when averaged over a family. These orthogonality relations are used to identify the main terms in the mean values.

In this section we give a detailed account of the case of moments of a single primitive $L$-function. We describe the recipe for conjecturing the mean values, applying it first to the case of the Riemann $\zeta$-function, and then to a general primitive $L$-function. In the remainder of this section we manipulate the formulas into a more usable form, and also obtain a generalization of Conjecture 1.5.1. Later in Section 3 we recast our principles in a more general setting and consider the averages of various families of $L$-functions.

2.1. The recipe. The following is our recipe for conjecturing the $2 k$ th moment of an $L$ function:

(1) Start with a product of $2 k$ shifted $L$-functions:

$$
Z\left(s, \alpha_{1}, \ldots, \alpha_{2 k}\right)=Z\left(\frac{1}{2}+\alpha_{1}\right) \cdots Z\left(\frac{1}{2}+\alpha_{2 k}\right)
$$

(here we have written the $Z$-function, but the examples below will show that the method applies to either the $L$ - or the $Z$-function).

(2) Replace each $L$-function with the two terms from its approximate functional equation, ignoring the remainder term. Multiply out the resulting expression to obtain $2^{2 k}$ terms.

(3) Keep the $\left(\begin{array}{c}2 k \\ k\end{array}\right)$ terms for which the product of $\chi$-factors from the functional equation is not rapidly oscillating. Use (2.2.9) to simplify the nonoscillating $\chi$-factors.

(4) In each of those $\left(\begin{array}{c}2 k \\ k\end{array}\right)$ terms, keep only the diagonal from the sum.

(5) Complete the resulting sums, and call the total $M\left(s, \alpha_{1}, \ldots, \alpha_{2 k}\right)$.

(6) The conjecture is

$$
\int_{-\infty}^{\infty} Z\left(\frac{1}{2}+i t, \alpha_{1}, \ldots, \alpha_{2 k}\right) g(t) d t=\int_{-\infty}^{\infty} M\left(\frac{1}{2}+i t, \alpha_{1}, \ldots, \alpha_{2 k}\right)\left(1+O\left(t^{-\frac{1}{2}+\varepsilon}\right)\right) g(t) d t
$$

for all $\varepsilon>0$, where $g$ is a suitable weight function. In other words, $L(s, \alpha)$ and $M(s, \alpha)$ have the same expected value if averaged over a sufficiently large range.

Notes:

(1) In order to see the structure of these mean values, it is necessary to include the shifts $\alpha_{j}$. One can obtain the moments of $L\left(\frac{1}{2}+i t\right)$ by allowing the shifts to tend to 0 . Because of the shifts $\alpha_{j}$ we avoid higher-order poles in our expressions.

(2) The recipe applies to either the $L$-function or the $Z$-function, and we give examples of both cases. The $Z$-function case can be directly obtained from the $L$-function, although the reverse is not true in general.

(3) For the approximate functional equations in the recipe, one can ignore the range of summation because it will just be extended to infinity in the final step. 
(4) We do not define what is meant by a "suitable weight function", but it is acceptable to take $g(t)=g_{T}(t)=f(t / T)$ for a fixed integrable function $f$. In particular, one can take $f$ to be the characteristic function of the interval $[0,1]$, obtaining the mean value $\int_{0}^{T} Z\left(\frac{1}{2}+i t, \alpha\right) d t$. From this one can recover a fairly general weighted integral by partial integration.

(5) The error term $O\left(t^{-\frac{1}{2}+\varepsilon}\right)$ fits with known examples and numerical evidence. See Section 5 ,

(6) The above procedure is a recipe for conjecturing all of the main terms in the mean value of an $L$-function. It is not a heuristic, and the steps cannot be justified. In particular, some steps can throw away terms which are the same size as the main term, and other steps add main terms back in. Our conjecture is that all of those errors cancel.

2.2. Moments of the Riemann $\zeta$-function. We illustrate our recipe in the case of the Riemann zeta-function. In this section we consider the most familiar case of moments of $\zeta\left(\frac{1}{2}+i t\right)$. In Section 2.3 we relate this to moments of $Z\left(\frac{1}{2}+i t\right)$ and repeat the calculation for the $Z$-function of an arbitrary primitive $L$-function.

Consider

$$
Z(s, \alpha)=\zeta\left(s+\alpha_{1}\right) \cdots \zeta\left(s+\alpha_{k}\right) \zeta\left(1-s-\alpha_{k+1}\right) \cdots \zeta\left(1-s-\alpha_{2 k}\right),
$$

where $\alpha=\left(\alpha_{1}, \ldots, \alpha_{2 k}\right)$. Note that this is slightly different notation than given in (2.1.1). Our goal is a formula for

$$
\int_{-\infty}^{\infty} Z\left(\frac{1}{2}+i t, \alpha\right) g(t) d t
$$

For each $\zeta$-function we use the approximate functional equation

$$
\zeta(s)=\sum_{m} \frac{1}{m^{s}}+\chi(s) \sum_{n} \frac{1}{n^{1-s}}+\text { remainder } .
$$

Recall that we ignore the remainder term and the limits on the sums. Multiplying out the resulting expression we obtain $2^{2 k}$ terms, and the recipe tells us to keep those terms in which the product of $\chi$-factors is not oscillating rapidly.

If $s=z+i t$ with $z$ bounded (but not necessarily real) then

$$
\chi(s)=\left(\frac{t}{2 \pi}\right)^{\frac{1}{2}-s} e^{i t+\pi i / 4}\left(1+O\left(\frac{1}{t}\right)\right)
$$

and

$$
\chi(1-s)=\left(\frac{t}{2 \pi}\right)^{s-\frac{1}{2}} e^{-i t-\pi i / 4}\left(1+O\left(\frac{1}{t}\right)\right),
$$

as $t \rightarrow+\infty$. We use the above formulas to determine which products of $\chi(s)$ and $\chi(1-s)$ are oscillating.

One term which does not have an oscillating factor is the one where we use the "first part" of each approximate functional equation, for it does not have any $\chi$-factors. With 
$s=\frac{1}{2}+i t$, that term is

$$
\begin{aligned}
\sum_{\substack{m_{1}, \ldots, m_{k} \\
n_{1}, \ldots, n_{k}}} m_{1}^{-s-\alpha_{1}} \cdots & m_{k}^{-s-\alpha_{k}} n_{1}^{s-1+\alpha_{k+1}} \cdots n_{k}^{s-1+\alpha_{2 k}} \\
& =\sum_{\substack{m_{1}, \ldots, m_{k} \\
n_{1}, \ldots, n_{k}}} m_{1}^{-\frac{1}{2}-\alpha_{1}} \cdots m_{k}^{-\frac{1}{2}-\alpha_{k}} n_{1}^{-\frac{1}{2}+\alpha_{k+1}} \cdots n_{k}^{-\frac{1}{2}+\alpha_{2 k}}\left(\frac{n_{1} \cdots n_{k}}{m_{1} \cdots m_{k}}\right)^{i t} .
\end{aligned}
$$

According to the recipe we keep the diagonal from the above sum, which is

$$
\sum_{m_{1} \cdots m_{k}=n_{1} \cdots n_{k}} m_{1}^{-\frac{1}{2}-\alpha_{1}} \cdots m_{k}^{-\frac{1}{2}-\alpha_{k}} n_{1}^{-\frac{1}{2}+\alpha_{k+1}} \cdots n_{k}^{-\frac{1}{2}+\alpha_{2 k}} .
$$

If we define

$$
R(s ; \alpha)=\sum_{m_{1} \cdots m_{k}=n_{1} \cdots n_{k}} \frac{1}{m_{1}^{s+\alpha_{1}} \cdots m_{k}^{s+\alpha_{k}} n_{1}^{s-\alpha_{k+1}} \cdots n_{k}^{s-\alpha_{2 k}}},
$$

where the sum is over all positive $m_{1}, \ldots, m_{k}, n_{1}, \ldots, n_{k}$ such that $m_{1} \cdots m_{k}=n_{1} \cdots n_{k}$, then $R\left(\frac{1}{2} ; \alpha\right)$ is the first piece which we have identified as contributing to the mean value. (The sum in equation (2.2.8) does not converge for $s=\frac{1}{2}$. See Theorem 2.4.1] for its analytic continuation.)

Note that the variable $s$ in equation (2.2.8) should not be viewed the same as the variable $s=\frac{1}{2}+i t$ from the previous equations. We are employing a trick of beginning with an expression involving $s$ and $1-s$, noting that we will later be setting $s=\frac{1}{2}$, so instead we consider an expression only involving $s$, which later will be set equal to $\frac{1}{2}$. This same trick will appear in Section 4.1 when we consider more general mean values.

Now consider one of the other terms, say the one where we use the second part of the approximate functional equation from $\zeta\left(s+\alpha_{1}\right)$ and the second part from $\zeta\left(1-s-\alpha_{k+1}\right)$. By (2.2.4) and (2.2.5),

$$
\chi\left(s+\alpha_{1}\right) \chi\left(1-s-\alpha_{k+1}\right) \sim\left(\frac{t}{2 \pi}\right)^{-\alpha_{1}+\alpha_{k+1}},
$$

which is not rapidly oscillating. Using this and proceeding as above, the contribution from this term will be

$$
\left(\frac{t}{2 \pi}\right)^{-\alpha_{1}+\alpha_{k+1}} R\left(\frac{1}{2} ; \alpha_{k+1}, \alpha_{2}, \ldots, \alpha_{k}, \alpha_{1}, \alpha_{k+2}, \ldots, \alpha_{2 k}\right) .
$$

More generally, note that

$\chi\left(s+\beta_{1}\right) \cdots \chi\left(s+\beta_{J}\right) \chi\left(1-s-\gamma_{1}\right) \cdots \chi\left(1-s-\gamma_{K}\right) \sim\left(\frac{t}{2 \pi e}\right)^{-i(J-K) t} e^{i(J-K) \pi / 4}\left(\frac{t}{2 \pi}\right)^{-\sum \beta_{j}+\sum \gamma_{j}}$,

which is rapidly oscillating (because of the it in the exponent) unless $J=K$. Thus, the recipe tell us to keep those terms which involve an equal number of $\chi\left(s+\alpha_{j}\right)$ and $\chi\left(1-s-\alpha_{k+j}\right)$ factors. This gives a total of $\left(\begin{array}{c}2 k \\ k\end{array}\right)=\sum_{j=0}^{k}\left(\begin{array}{c}k \\ j\end{array}\right)^{2}$ terms in the final answer.

We now describe a typical term of the conjectural formula. First note that the function $R\left(s ; \alpha_{1}, \ldots, \alpha_{k}, \alpha_{k+1}, \ldots, \alpha_{2 k}\right)$ is symmetric in $\alpha_{1}, \ldots, \alpha_{k}$ and in $\alpha_{k+1}, \ldots, \alpha_{2 k}$, so we can rearrange the entries so that the first $k$ are in increasing order, as are the last $k$. Thus, 
the final result will be a sum of terms indexed by the $\left(\begin{array}{c}2 k \\ k\end{array}\right)$ permutations $\sigma \in S_{2 k}$ such that $\sigma(1)<\cdots<\sigma(k)$ and $\sigma(k+1)<\cdots<\sigma(2 k)$. We denote the set of such permutations by $\Xi$. Second, note that the product of an equal number of $\chi\left(s+\alpha_{j}\right)$ and $\chi\left(1-s-\alpha_{k+j}\right)$, as in (2.2.9), can be written as

$$
\left(\frac{t}{2 \pi}\right)^{\frac{1}{2}\left(-\alpha_{1}-\cdots-\alpha_{k}+\alpha_{k+1}+\cdots+\alpha_{2 k}\right)}\left(\frac{t}{2 \pi}\right)^{\frac{1}{2}\left(\alpha_{\sigma(1)}+\cdots+\alpha_{\sigma(k)}-\alpha_{\sigma(k+1)}-\cdots-\alpha_{\sigma(2 k)}\right)} .
$$

For example, (2.2.9) is the case $\sigma(1)=k+1, \sigma(k+1)=1$, and $\sigma(j)=j$ otherwise.

If we set

$$
W(z, \alpha, \sigma)=\left(\frac{y}{2 \pi}\right)^{\frac{1}{2}\left(\alpha_{\sigma(1)}+\cdots+\alpha_{\sigma(k)}-\alpha_{\sigma(k+1)}-\cdots-\alpha_{\sigma(2 k)}\right)} R\left(x ; \alpha_{\sigma(1)}, \ldots, \alpha_{\sigma(2 k)}\right),
$$

for $z=x+i y$ with $x$ and $y$ real, then combining all terms we have

$$
M(z ; \alpha):=\left(\frac{y}{2 \pi}\right)^{\frac{1}{2}\left(-\alpha_{1}-\cdots-\alpha_{k}+\alpha_{k+1}+\cdots+\alpha_{2 k}\right)} \sum_{\sigma \in \Xi} W(z, \alpha, \sigma) .
$$

The recipe has produced the conjecture

$$
\int_{-\infty}^{\infty} Z\left(\frac{1}{2}+i t, \alpha\right) g(t) d t=\int_{-\infty}^{\infty} M\left(\frac{1}{2}+i t, \alpha\right)\left(1+O\left(t^{-\frac{1}{2}+\varepsilon}\right)\right) g(t) d t
$$

with $Z(s, \alpha)$ given in (2.2.1) and $M(s ; \alpha)$ given above.

Note that the exponent of $(t / 2 \pi)$ in (2.2.13) has half the $\alpha_{j}$ with + sign and the other half with - sign, and the same holds for $R(s, \alpha)$. This allows an alternate interpretation of $\Xi$ as the set of ways of choosing $k$ elements from $\left\{\alpha_{1}, \ldots, \alpha_{2 k}\right\}$.

The general case of Conjecture 1.5.1 is stated in terms of the $Z$-function. We can recover the mean value of the $Z$-function directly from that of the $L$-function (in this case, the $\zeta$ function). By the functional equation and (2.2.4) we see that

$$
\begin{aligned}
Z\left(s+\alpha_{1}\right) \cdots Z\left(s+\alpha_{2 k}\right)= & \left(\frac{t}{2 \pi}\right)^{\frac{1}{2}\left(\alpha_{1}+\cdots+\alpha_{k}-\alpha_{k+1}-\cdots-\alpha_{2 k}\right)}(1+O(1 / t)) \\
2.2 .16) & \times \zeta\left(s+\alpha_{1}\right) \cdots \zeta\left(s+\alpha_{k}\right) \zeta\left(1-s-\alpha_{k+1}\right) \cdots \zeta\left(1-s-\alpha_{2 k}\right) .
\end{aligned}
$$

The factor $\left(\frac{t}{2 \pi}\right)^{\frac{1}{2}\left(\alpha_{1}+\cdots+\alpha_{k}-\alpha_{k+1}-\cdots-\alpha_{2 k}\right)}$ can be absorbed into the weight function $g(t)$, so we obtain the conjecture

$$
\int_{-\infty}^{\infty} Z\left(s+\alpha_{1}\right) \cdots Z\left(s+\alpha_{2 k}\right) g(t) d t=\int_{-\infty}^{\infty} \sum_{\sigma \in \Xi} W(s, \alpha, \sigma)\left(1+O\left(t^{-\frac{1}{2}+\varepsilon}\right)\right) g(t) d t
$$

where $s=\frac{1}{2}+i t$.

In the next subsection we directly obtain the above conjecture for the $Z$-function of a general primitive $L$-function, and in the remainder of this section we perform various manipulations to put these in the form of Conjecture 1.5.1. 
2.3. Moments of a primitive $L$-function. Consider the primitive $L$-function

$$
\mathcal{L}(s)=\sum_{n=1}^{\infty} \frac{a_{n}}{n^{s}}=\prod_{p} \mathcal{L}_{p}\left(\frac{1}{p^{s}}\right), \quad(\sigma>1) .
$$

We assume a functional equation of the special form $\xi_{L}(s)=\gamma_{L}(s) L(s)=\varepsilon \bar{\xi}_{L}(1-s)$, where

$$
\gamma_{L}(s)=Q^{s} \prod_{j=1}^{w} \Gamma\left(\frac{1}{2} s+\mu_{j}\right)
$$

with $\left\{\mu_{j}\right\}$ stable under complex conjugation. Note that we have $w_{j}=\frac{1}{2}$, which is conjectured to hold for arithmetic $L$-functions. We also assume

$$
\begin{aligned}
\mathcal{L}_{p}(x) & =\sum_{n=0}^{\infty} a_{p^{n}} x^{n} \\
& =\prod_{j=1}^{w}\left(1-\gamma_{p, j} x\right)^{-1},
\end{aligned}
$$

where $w$ is the degree of $\mathcal{L}$ and where $\left|\gamma_{p, j}\right|=0$ or 1 . Again this is conjectured to hold for arithmetic $L$-functions.

We are going to evaluate the moments of the $Z$-function $Z_{\mathcal{L}}(s)=\varepsilon^{-\frac{1}{2}} \mathcal{X}(s)^{-\frac{1}{2}} \mathcal{L}(s)$, where

$$
\begin{aligned}
\mathcal{X}(s) & =\frac{\overline{\gamma_{\mathcal{L}}(1-s)}}{\gamma_{\mathcal{L}}(s)} \\
& =Q^{1-2 s} \prod_{j=1}^{w} \frac{\Gamma\left(\frac{1}{2}(1-s)+\overline{\mu_{j}}\right)}{\Gamma\left(\frac{1}{2} s+\mu_{j}\right)} .
\end{aligned}
$$

We will have to determine when products of $\mathcal{X}(s)$ and $\mathcal{X}(1-s)$ are not rapidly oscillating. By Stirling's formula

$$
\Gamma(\sigma+i t)=e^{-\frac{\pi t}{2}} t^{\sigma-\frac{1}{2}}\left(\frac{t}{e}\right)^{i t} e^{\frac{i \pi}{2}\left(\sigma-\frac{1}{2}\right)}\left(1-\frac{i}{t}\left(\frac{1}{12}-\frac{\sigma}{2}+\frac{\sigma^{2}}{2}\right)+O\left(\frac{1}{t^{2}}\right)\right)
$$

we obtain

$$
\mathcal{X}(s)=Q^{1-2 s}\left(\frac{t}{2}\right)^{w\left(\frac{1}{2}-s\right)}\left(\frac{t}{2 \pi e}\right)^{-\sum \Im\left(\mu_{j}\right)} e^{w(i t+i \pi / 4)}\left(1+O\left(\frac{1}{t}\right)\right),
$$

as $t \rightarrow+\infty$. Note that the above expression can be simplified because we have assumed $\sum \Im\left(\mu_{j}\right)=0$.

Now we are ready to produce a conjecture for

$$
I_{k}\left(\mathcal{L}, \alpha_{1}, \ldots, \alpha_{2 k}, g\right)=\int_{-\infty}^{\infty} Z_{\mathcal{L}}\left(s+\alpha_{1}\right) \cdots Z_{\mathcal{L}}\left(s+\alpha_{2 k}\right) g(t) d t
$$

with $s=\frac{1}{2}+i t$.

By the definition of $Z$,

$$
\prod_{j=1}^{2 k} Z_{\mathcal{L}}\left(s+\alpha_{j}\right)=\prod_{j=1}^{2 k} \varepsilon^{-\frac{1}{2}} \mathcal{X}\left(s+\alpha_{j}\right)^{-\frac{1}{2}} \prod_{j=1}^{2 k} \mathcal{L}\left(s+\alpha_{j}\right) .
$$


According to the recipe, we replace each $\mathcal{L}(s+i t)$ by its approximate functional equation and multiply out the product obtaining $2^{2 k}$ terms. A typical term is a product of $2 k$ sums arising from either the first piece or the second piece of the approximate functional equation. Consider a term where we have $\ell$ factors from the first piece of the approximate functional equation and $2 k-\ell$ factors from the second piece. To take one specific example, suppose it is the first $\ell$ factors where we choose the first piece of the approximate functional equation, and the last $2 k-\ell$ factors where we take the second piece:

$$
\begin{aligned}
\varepsilon^{-k} \mathcal{X}\left(\frac{1}{2}+\right. & \left.\alpha_{1}+i t\right)^{-\frac{1}{2}} \cdots \mathcal{X}\left(\frac{1}{2}+\alpha_{\ell}+i t\right)^{-\frac{1}{2}} \sum_{n_{1}} \frac{a_{n_{1}}}{n_{1}^{\frac{1}{2}+\alpha_{1}+i t}} \cdots \sum_{n_{\ell}} \frac{a_{n_{\ell}}}{n_{\ell}^{\frac{1}{2}+\alpha_{\ell}+i t}} \\
& \times \varepsilon^{2 k-\ell} \mathcal{X}\left(\frac{1}{2}+\alpha_{\ell+1}+i t\right)^{\frac{1}{2}} \cdots \mathcal{X}\left(\frac{1}{2}+\alpha_{2 k}+i t\right)^{\frac{1}{2}} \sum_{n_{\ell+1}} \frac{\overline{a_{n_{\ell+1}}}}{n_{\ell+1}^{\frac{1}{2}-\alpha_{\ell+1}-i t}} \cdots \sum_{n_{2 k}} \frac{\overline{a_{n_{2 k}}}}{n_{2 k}^{\frac{1}{2}-\alpha_{2 k}-i t}} \\
=\varepsilon^{k-\ell}( & \left.\frac{\mathcal{X}\left(\frac{1}{2}+\alpha_{1}+i t\right) \cdots \mathcal{X}\left(\frac{1}{2}+\alpha_{\ell}+i t\right)}{\mathcal{X}\left(\frac{1}{2}+\alpha_{\ell+1}+i t\right) \cdots \mathcal{X}\left(\frac{1}{2}+\alpha_{2 k}+i t\right)}\right)^{-\frac{1}{2}} \\
& \times \sum_{n_{1}} \cdots \sum_{n_{2 k}} \frac{a_{n_{1}} \cdots a_{n_{\ell}} \overline{a_{n_{\ell+1}} \cdots a_{n_{k}}}}{n_{1}^{\frac{1}{2}+\alpha_{1}} \cdots n_{\ell}^{\frac{1}{2}+\alpha_{\ell}} n_{\ell+1}^{\frac{1}{2}-\alpha_{\ell+1}} \cdots n_{2 k}^{\frac{1}{2}-\alpha_{2 k}}}\left(\frac{n_{1} \cdots n_{\ell}}{n_{\ell+1} \cdots n_{2 k}}\right)^{-i t} .
\end{aligned}
$$

The recipe tells us to retain only the expressions of this sort where the factor involving $\mathcal{X}$ is not oscillating. By (2.3.6) the requirement is that $\ell=k$ (and in particular $2 k$ has to be even), and we have

$$
\left(\frac{\mathcal{X}\left(\frac{1}{2}+\alpha_{1}+i t\right) \ldots \mathcal{X}\left(\frac{1}{2}+\alpha_{k}+i t\right)}{\mathcal{X}\left(\frac{1}{2}+\alpha_{k+1}+i t\right) \ldots \mathcal{X}\left(\frac{1}{2}+\alpha_{2 k}+i t\right)}\right)^{-\frac{1}{2}}=\left(\frac{Q^{\frac{2}{w}} t}{2}\right)^{\frac{w}{2}\left(\alpha_{1}+\cdots+\alpha_{k}-\alpha_{k+1}-\cdots-\alpha_{2 k}\right)}\left(1+O\left(\frac{1}{t}\right)\right)
$$

Now the recipe tells us to keep the diagonal from the remaining sums, which in (2.3.9) is the terms where $n_{1} \cdots n_{\ell}=n_{\ell+1} \cdots n_{2 k}$. So in the same way as the $\zeta$-function case in the previous section we let

$$
R(s, \alpha)=\sum_{n_{1} \cdots n_{k}=n_{k+1} \cdots n_{2 k}} \frac{a_{n_{1}} \cdots a_{n_{k}} \overline{a_{n_{k+1}} \cdots a_{n_{k}}}}{n_{1}^{s+\alpha_{1}} \cdots n_{k}^{s+\alpha_{k}} n_{k+1}^{s-\alpha_{k+1}} \cdots n_{2 k}^{s-\alpha_{2 k}}},
$$

and

$$
W(z, \alpha, \sigma)=\left(\frac{Q^{\frac{2}{w}} y}{2}\right)^{\frac{w}{2}\left(\alpha_{\sigma(1)}+\cdots+\alpha_{\sigma(k)}-\alpha_{\sigma(k+1)}-\cdots-\alpha_{\sigma(2 k)}\right)} R\left(x ; \alpha_{\sigma(1)}, \ldots, \alpha_{\sigma(2 k)}\right),
$$

for $\sigma \in \Xi$, the set of permutation of $\{1, \ldots, 2 k\}$ with $\sigma(1)<\cdots<\sigma(k)$ and $\sigma(k+1)<\cdots<$ $\sigma(2 k)$. Then

$$
M(z ; \alpha)=\sum_{\sigma \in \Xi} W(z, \alpha, \sigma)
$$

and we arrive at the conjecture

$$
I_{k}\left(\mathcal{L}, \alpha_{1}, \ldots, \alpha_{2 k}, g\right)=\int_{-\infty}^{\infty} M\left(\frac{1}{2}+i t, \alpha\right)\left(1+O\left(t^{-\frac{1}{2}+\varepsilon}\right)\right) g(t) d t
$$

which is the same form as we obtained in (2.2.17). 
We will now examine the expressions in these conjectures in detail, rewriting them in a more explicit form.

2.4. The arithmetic factor in the conjectures. We retain the notation of the previous subsection. In particular, $\mathcal{L}(s)$ is a primitive $L$-function having the properties listed at the beginning of Section 2.3 and $R(s ; \alpha)$ is given in (2.3.11).

Theorem 2.4.1. Suppose $\left|\alpha_{j}\right|<\delta$ for $j=1, \ldots, 2 k$. Then $R\left(s ; \alpha_{1}, \ldots, \alpha_{2 k}\right)$ converges absolutely for $\sigma>\frac{1}{2}+\delta$ and has a meromorphic continuation to $\sigma>\frac{1}{4}+\delta$. Furthermore,

$$
R\left(s ; \alpha_{1}, \ldots, \alpha_{2 k}\right)=\prod_{i, j=1}^{k} \zeta\left(2 s+\alpha_{i}-\alpha_{k+j}\right) A_{k}\left(s ; \alpha_{1}, \ldots, \alpha_{2 k}\right)
$$

where

$$
A_{k}\left(s ; \alpha_{1}, \ldots, \alpha_{2 k}\right)=\prod_{p}\left(\prod_{i, j=1}^{k}\left(1-p^{-2 s-\alpha_{i}+\alpha_{k+j}}\right)\right) B_{p}\left(s ; \alpha_{1}, \ldots, \alpha_{2 k}\right)
$$

with

$$
B_{p}\left(s ; \alpha_{1}, \ldots, \alpha_{2 k}\right)=\int_{0}^{1} \prod_{j=1}^{k} \mathcal{L}_{p}\left(\frac{e(\theta)}{p^{s+\alpha_{j}}}\right) \overline{\mathcal{L}_{p}}\left(\frac{e(-\theta)}{p^{s-\alpha_{k+j}}}\right) d \theta
$$

Proof. We assumed $\left|\gamma_{p, j}\right| \leq 1$, so we have the Ramanujan bound $a_{n} \leq d_{w}(n) \ll n^{\varepsilon}$. That implies absolute convergence of $R(s ; \alpha)$ for $\sigma>\frac{1}{2}+\delta+\varepsilon$.

Since the coefficients of $R(s ; \alpha)$ are multiplicative, as is the condition $n_{1} \cdots n_{k}=n_{k+1} \cdots n_{2 k}$, we can write $R(s ; \alpha)$ as an Euler product:

$$
\begin{aligned}
& R\left(s ; \alpha_{1}, \ldots, \alpha_{2 k}\right)=\sum_{n_{1} \cdots n_{k}=n_{k+1} \cdots n_{2 k}} \frac{a_{n_{1}} \cdots a_{n_{k}} \overline{a_{n_{k+1}} \cdots a_{n_{2 k}}}}{n_{1}^{s+\alpha_{1}} \cdots n_{k}^{s-\alpha_{2 k}}} \\
& =\prod_{p} \sum_{\sum_{j=1}^{k} e_{j}=\sum_{j=1}^{k} e_{k+j}} \frac{a_{p^{e_{1}}} \cdots a_{p_{k} e_{k}} \overline{a_{p^{e} k+1} \cdots a_{p^{e_{2 k}}}}}{p^{e_{1}\left(s+\alpha_{1}\right)} \cdots p^{e_{2 k}\left(s-\alpha_{2 k}\right)}} \\
& =\prod_{p}\left(1+\left|a_{p}\right|^{2} \sum_{i, j=1}^{k} \frac{1}{p^{2 s+\alpha_{i}-\alpha_{k+j}}}+\sum_{j=2}^{\infty} \frac{c_{p^{j}}\left(\alpha_{1}, \ldots, \alpha_{2 k}\right)}{p^{2 j s}}+\cdots\right) \\
& =\prod_{i, j=1}^{k} \zeta\left(2 s+\alpha_{i}-\alpha_{k+j}\right) \\
& \times \prod_{p}\left(1+\left(\left|a_{p}\right|^{2}-1\right) \sum_{i, j=1}^{k} \frac{1}{p^{2 s+\alpha_{i}-\alpha_{k+j}}}+\sum_{j=2}^{\infty} \frac{c_{p^{j}}^{\prime}\left(\alpha_{1}, \ldots, \alpha_{2 k}\right)}{p^{2 j s}}+\cdots\right) \\
& =\prod_{i, j=1}^{k} \zeta\left(2 s+\alpha_{i}-\alpha_{k+j}\right) A_{k}\left(s ; \alpha_{1}, \ldots, \alpha_{2 k}\right),
\end{aligned}
$$

say. Above $c_{p^{j}}$ and $c_{p^{j}}^{\prime}$ are just shorthand for the (complicated) coefficients in the Euler product. Estimating them trivially and using the fact that $\left|a_{p}\right|^{2}$ is 1 on average, (which 
is conjectured to hold for primitive elements of the Selberg class) we find that $A_{k}(s ; \alpha)$ is analytic in a neighborhood of $\sigma=\frac{1}{2}$.

Finally, we have

$$
A_{k}\left(s ; \alpha_{1}, \ldots, \alpha_{2 k}\right)=\prod_{p} \prod_{i, j=1}^{k}\left(1-p^{-2 s-\alpha_{i}+\alpha_{k+j}}\right) B_{p}\left(s ; \alpha_{1}, \ldots, \alpha_{2 k}\right)
$$

where

$$
\begin{aligned}
& B_{p}\left(s ; \alpha_{1}, \ldots, \alpha_{2 k}\right)=\sum_{\sum_{j=1}^{k} e_{j}=\sum_{j=1}^{k} e_{k+j}} \frac{a_{p^{e_{1}}} \cdots a_{p^{e_{k}}} \overline{a_{p^{e_{k+1}}} \cdots a_{p^{e_{2 k}}}}}{p^{e_{1}\left(s+\alpha_{1}\right) \cdots p^{e_{2 k}}\left(s-\alpha_{2 k}\right)}} \\
& =\int_{0}^{1} \sum_{e_{1}, \ldots, e_{2 k}} \frac{a_{p^{e_{1}}} \cdots a_{p^{e_{k}}} \overline{a_{p^{e_{k+1}}} \cdots a_{p^{e_{2 k}}}}}{p^{e_{1}\left(s+\alpha_{1}\right)} \cdots p^{e_{2 k}\left(s-\alpha_{2 k}\right)}} e\left(\left(\sum_{j=1}^{k} e_{j}-\sum_{j=1}^{k} e_{k+j}\right) \theta\right) d \theta \\
& =\int_{0}^{1} \prod_{j=1}^{k} \sum_{e_{j}=0}^{\infty} \frac{a_{p^{e_{j}}}}{p^{e_{j}\left(s+\alpha_{j}\right)}} e\left(e_{j} \theta\right) \prod_{j=1}^{k} \frac{\overline{a_{p} e_{k+j}}}{p^{e_{k+j}\left(s-\alpha_{k+j}\right)}} e\left(-e_{k+j} \theta\right) d \theta \\
& =\int_{0}^{1} \prod_{j=1}^{k} \mathcal{L}_{p}\left(\frac{e(\theta)}{p^{s+\alpha_{j}}}\right) \overline{\mathcal{L}_{p}}\left(\frac{e(-\theta)}{p^{s-\alpha_{k+j}}}\right) d \theta
\end{aligned}
$$

as claimed.

To summarize, the conjecture for the general mean value $I_{k}\left(\mathcal{L}, \alpha_{1}, \ldots, \alpha_{2 k}, g\right)$ involves the function $M(s ; \alpha)$, which can be written as

$$
M(s ; \alpha)=\sum_{\sigma \in \Xi} W\left(s ; \alpha_{\sigma(1)}, \ldots, \alpha_{\sigma(2 k)}\right),
$$

where we have written $W\left(s ; \alpha_{\sigma(1)}, \ldots, \alpha_{\sigma(2 k)}\right)$ for $W(s ; \alpha)$. And by Theorem 2.4.1.

$$
W\left(s ; \alpha_{1}, \ldots, \alpha_{2 k}\right)=\left(\frac{Q^{\frac{2}{w}} t}{2}\right)^{\frac{w}{2} \sum_{j=1}^{k} \alpha_{j}-\alpha_{k+j}} A_{k}\left(s ; \alpha_{1}, \ldots, \alpha_{2 k}\right) \prod_{i, j=1}^{k} \zeta\left(2 s+\alpha_{i}-\alpha_{k+j}\right)
$$

One can see the above elements in Conjecture 1.5.1, in particular the form of $B_{p}(s ; \alpha)$, and $A_{k}(z)$ in that conjecture equals $A_{k}\left(\frac{1}{2}, \alpha\right)$ given above. The overall structure is slightly different because Conjecture 1.5.1 is expressed as a multiple contour integral, as opposed to a sum over permutations. In the next subsection we show how to write the sum over permutations in a compact form. In the following subsection we return to the functions $A_{k}$ and write them in a more explicit form.

2.5. Concise form of permutation sums. As we have seen, our methods naturally lead to an expression involving a sum over permutations. In this section we describe how to write those sums in a compact form involving contour integrals. Similar combinatorial sums arise from our matrix ensemble calculations, and we have previously stated our main results and conjectures in this compact form.

Note that in both of these Lemmas, the terms in the sum on the left side have singularities. However, examining the right side of the formula makes it clear that those singularities all cancel. 
Lemma 2.5.1. Suppose $F(a ; b)=F\left(a_{1}, \ldots, a_{k} ; b_{1}, \ldots, b_{k}\right)$ is a function of $2 k$ variables, which is symmetric with respect to the first $k$ variables and also symmetric with respect to the second set of $k$ variables. Suppose also that $F$ is regular near $(0, \ldots, 0)$. Suppose further that $f(s)$ has a simple pole of residue 1 at $s=0$ but is otherwise analytic in a neighborhood about $s=0$. Let

$$
K\left(a_{1}, \ldots, a_{k} ; b_{1}, \ldots b_{k}\right)=F\left(a_{1}, \ldots ; \ldots, b_{k}\right) \prod_{i=1}^{k} \prod_{j=1}^{k} f\left(a_{i}-b_{j}\right) .
$$

If for all $1 \leq i, j \leq k, \alpha_{i}-\alpha_{k+j}$ is contained in the region of analyticity of $f(s)$ then

$$
\sum_{\sigma \in \Xi}
$$

$$
\begin{aligned}
& K\left(\alpha_{\sigma(1)}, \ldots, \alpha_{\sigma(k)} ; \alpha_{\sigma(k+1)} \ldots \alpha_{\sigma(2 k)}\right)= \\
& \quad \frac{(-1)^{k}}{k !^{2}} \frac{1}{(2 \pi i)^{2 k}} \oint \ldots \oint \frac{K\left(z_{1}, \ldots, z_{k} ; z_{k+1}, \ldots, z_{2 k}\right) \Delta\left(z_{1}, \ldots, z_{2 k}\right)^{2}}{\prod_{i=1}^{2 k} \prod_{j=1}^{2 k}\left(z_{i}-\alpha_{j}\right)} d z_{1} \ldots d z_{2 k},
\end{aligned}
$$

where one integrates about small circles enclosing the $\alpha_{j}$ 's, and where $\Xi$ is the set of $\left(\begin{array}{c}2 k \\ k\end{array}\right)$ permutations $\sigma \in S_{2 k}$ such that $\sigma(1)<\cdots<\sigma(k)$ and $\sigma(k+1)<\cdots<\sigma(2 k)$.

The above Lemma applies to the Unitary case, which has been the subject of this section. The next Lemma is useful in the Symplectic and Orthogonal cases, which will be addressed beginning in Section 4.4

Lemma 2.5.2. Suppose $F$ is a symmetric function of $k$ variables, regular near $(0, \ldots, 0)$, and $f(s)$ has a simple pole of residue 1 at $s=0$ and is otherwise analytic in a neighborhood of $s=0$, and let

$$
K\left(a_{1}, \ldots, a_{k}\right)=F\left(a_{1}, \ldots, a_{k}\right) \prod_{1 \leq i \leq j \leq k} f\left(a_{i}+a_{j}\right)
$$

or

$$
K\left(a_{1}, \ldots, a_{k}\right)=F\left(a_{1}, \ldots, a_{k}\right) \prod_{1 \leq i<j \leq k} f\left(a_{i}+a_{j}\right) .
$$

If $\alpha_{i}+\alpha_{j}$ are contained in the region of analyticity of $f(s)$ then

$$
\sum_{\epsilon_{j}= \pm 1} K\left(\epsilon_{1} \alpha_{1}, \ldots, \epsilon_{k} \alpha_{k}\right)=
$$

$$
\frac{(-1)^{k(k-1) / 2}}{(2 \pi i)^{k}} \frac{2^{k}}{k !} \oint \cdots \oint K\left(z_{1}, \ldots, z_{k}\right) \frac{\Delta\left(z_{1}^{2}, \ldots, z_{k}^{2}\right)^{2} \prod_{j=1}^{k} z_{j}}{\prod_{i=1}^{k} \prod_{j=1}^{k}\left(z_{i}-\alpha_{j}\right)\left(z_{i}+\alpha_{j}\right)} d z_{1} \cdots d z_{k}
$$

and

$$
\sum_{\epsilon_{j}= \pm 1}\left(\prod_{j=1}^{k} \epsilon_{j}\right) K\left(\epsilon_{1} \alpha_{1}, \ldots, \epsilon_{k} \alpha_{k}\right)=
$$

$$
\frac{(-1)^{k(k-1) / 2}}{(2 \pi i)^{k}} \frac{2^{k}}{k !} \oint \cdots \oint K\left(z_{1}, \ldots, z_{k}\right) \frac{\Delta\left(z_{1}^{2}, \ldots, z_{k}^{2}\right)^{2} \prod_{j=1}^{k} \alpha_{j}}{\prod_{i=1}^{k} \prod_{j=1}^{k}\left(z_{i}-\alpha_{j}\right)\left(z_{i}+\alpha_{j}\right)} d z_{1} \cdots d z_{k},
$$

where the path of integration encloses the $\pm \alpha_{j}$ 's.

The proofs of the lemmas come from the following 
Lemma 2.5.3. Suppose that $F(a ; b)=F\left(a_{1}, \ldots, a_{m} ; b_{1}, \ldots, b_{n}\right)$ is symmetric in the a variables and in the $b$ variables and is regular near $(0, \ldots, 0)$. Suppose $f(s)=\frac{1}{s}+c+\cdots$ and let

$$
G\left(a_{1}, \ldots, a_{m} ; b_{1}, \ldots b_{n}\right)=F\left(a_{1}, \ldots ; \ldots, b_{n}\right) \prod_{i=1}^{m} \prod_{j=1}^{n} f\left(a_{i}-b_{j}\right) .
$$

Let $\Xi_{m, n}$ be as defined above. Then

$$
\begin{array}{rl}
\sum_{\sigma \in \Xi_{m, n}} & G\left(\alpha_{\sigma(1)}, \ldots, \alpha_{\sigma(m)} ; \alpha_{\sigma(m+1)} \ldots \alpha_{\sigma(m+n)}\right)= \\
& \frac{(-1)^{(m+n)}}{m ! n !} \sum_{\sigma \in \pi_{m+n}} \operatorname{Res}_{\left(z_{1}, \ldots, z_{m+n}\right)=\left(\alpha_{\sigma(1)}, \ldots, \alpha_{\sigma(m+n)}\right)} \frac{G\left(z_{1}, \ldots, z_{m+n}\right) \Delta\left(z_{1}, \ldots, z_{m+n}\right)^{2}}{\prod_{i=1}^{m+n} \prod_{j=1}^{m+n}\left(z_{i}-\alpha_{j}\right)} .
\end{array}
$$

Proof. It suffices to prove that

$$
\operatorname{Res}_{\left(z_{1}, \ldots, z_{m+n}\right)=\left(\alpha_{\sigma(1)}, \ldots, \alpha_{\sigma(m+n)}\right)} \frac{\Delta\left(z_{1}, \ldots, z_{m+n}\right)^{2}}{\prod_{i=1}^{m+n} \prod_{j=1}^{m+n}\left(z_{i}-\alpha_{j}\right)}=(-1)^{m+n}
$$

since each such term will appear $m ! n$ ! times. Consider the case where $\sigma$ is the identity permutation. Then the residue is

$$
\frac{\prod_{j<k}\left(\alpha_{k}-\alpha_{j}\right)^{2}}{\prod_{j \neq k}\left(\alpha_{j}-\alpha_{k}\right)}=(-1)^{m+n}
$$

the answer will be the same for any permutation $\sigma$.

The residue above can be expressed as $(2 \pi i)^{-m-n}$ times an $m+n$ fold integral, each path of which encircles all of the poles of the integrand; note that the value of such an integral may be calculated by summing the residues and note that there is no singularity when $z_{j}=z_{k}$ because of the factor $\left(z_{k}-z_{j}\right)^{2}$ in the numerator.

To obtain the form of Conjecture 1.5.1 from the formulas at the end of Sections 2.3 and [2.4 apply Lemma 2.5.1] with $K\left(\alpha_{1}, \ldots, \alpha_{2 k}\right)=W\left(\frac{1}{2} ; \alpha_{1}, \ldots, \alpha_{2 k}\right)$. That is, $f(z)=$ $\zeta(1+z)$ and

$$
\begin{aligned}
F\left(\alpha_{1}, \ldots, \alpha_{2 k}\right) & =\left(\frac{Q^{\frac{2}{w}} t}{2}\right)^{\frac{w}{2} \sum_{j=1}^{k} \alpha_{j}-\alpha_{k+j}} A_{k}\left(\frac{1}{2} ; \alpha_{1}, \ldots, \alpha_{2 k}\right) \\
& =\exp \left(w \log \left(\frac{Q^{\frac{2}{w}} t}{2}\right) \cdot \frac{1}{2} \sum_{j=1}^{k} \alpha_{j}-\alpha_{k+j}\right) A_{k}\left(\frac{1}{2} ; \alpha_{1}, \ldots, \alpha_{2 k}\right) .
\end{aligned}
$$

We arrive at the general case of Conjecture 1.5.1.

Conjecture 2.5.4. Suppose $\mathcal{L}(s)$ is a primitive L-function having the properties listed at the beginning of Section [2.3, and the mean value $I_{k}\left(\mathcal{L}, \alpha_{1}, \ldots, \alpha_{2 k}, g\right)$ is given in 2.3.7). Then

$$
I_{k}\left(\mathcal{L}, \alpha_{1}, \ldots, \alpha_{2 k}, g\right)=\int_{-\infty}^{\infty} P_{k}\left(w \log \left(\frac{Q^{\frac{2}{w}} t}{2}\right), \alpha\right)\left(1+O\left(t^{-\frac{1}{2}+\varepsilon}\right)\right) g(t) d t
$$


where $P_{k}(x, \alpha)$ and $G\left(z_{1}, \ldots, z_{2 k}\right)$ are as stated in Conjecture 1.5.1, except that $A_{k}$ is the Euler product

$$
A_{k}(z)=\prod_{p} \prod_{i=1}^{k} \prod_{j=1}^{k}\left(1-\frac{1}{p^{1+z_{i}-z_{k+j}}}\right) \int_{0}^{1} \prod_{j=1}^{k} \mathcal{L}_{p}\left(\frac{e(\theta)}{p^{\frac{1}{2}+z_{j}}}\right) \overline{\mathcal{L}_{p}}\left(\frac{e(-\theta)}{p^{\frac{1}{2}-z_{k+j}}}\right) .
$$

Note that for the Riemann $\zeta$-function, $w=1$ and $Q=1 / \sqrt{\pi}$ and $\mathcal{L}_{p}(x)=(1-x)^{-1}$, so Conjecture 1.5.1 is a special case of the above. Also note that $w \log \left(\frac{Q^{\frac{2}{w}} t}{2}\right)$ is the mean density of zeros of $\mathcal{L}\left(\frac{1}{2}+i t\right)$, or equivalently the log conductor, as expected.

It remains to express the arithmetic factor $A_{k}$ in a more explicit form, which we do in the next section.

2.6. Explicit versions of the arithmetic factor. The factor $A_{k}(s, \alpha)$ in the $2 k$ th moment of a primitive $L$-function can be expressed in a simple form.

Recall, see Theorem 2.4.1, that $A_{k}$ is the Euler product

$$
A_{k}(s ; \alpha)=\prod_{p} B_{p}\left(s ; \alpha_{1}, \ldots, \alpha_{2 k}\right) \prod_{i=1}^{k} \prod_{j=1}^{k}\left(1-\frac{1}{p^{2 s+\alpha_{i}-\alpha_{k+j}}}\right),
$$

where

$$
B_{p}\left(s ; \alpha_{1}, \ldots, \alpha_{2 k}\right)=\int_{0}^{1} \prod_{j=1}^{k} \mathcal{L}_{p}\left(\frac{e(\theta)}{p^{s+\alpha_{j}}}\right) \overline{\mathcal{L}_{p}}\left(\frac{e(-\theta)}{p^{s-\alpha_{k+j}}}\right) d \theta .
$$

Lemma 2.6.1. If $\mathcal{L}_{p}(x)=\left(1-\gamma_{p} x\right)^{-1}$ with $\left|\gamma_{p}\right|=1$ then

$$
B_{p}\left(s ; \alpha_{1}, \ldots, \alpha_{2 k}\right)=\prod_{i=1}^{k} \prod_{j=1}^{k}\left(1-\frac{1}{p^{2 s+\alpha_{i}-\alpha_{k+j}}}\right)^{-1} \sum_{m=1}^{k} \prod_{i \neq m} \frac{\prod_{j=1}^{k}\left(1-\frac{1}{p^{2 s+\alpha_{j}-\alpha_{k+i}}}\right)}{1-p^{\alpha_{k+i}-\alpha_{k+m}}} .
$$

Corollary 2.6.2. If $\mathcal{L}_{p}(x)=\left(1-\gamma_{p} x\right)^{-1}$ with $\left|\gamma_{p}\right|=0$ when $p \mid N$ and $\left|\gamma_{p}\right|=1$ otherwise, then

$$
A_{k}\left(s ; \alpha_{1}, \ldots, \alpha_{2 k}\right)=\prod_{p \nmid N} \sum_{m=1}^{k} \prod_{i \neq m} \frac{\prod_{j=1}^{k}\left(1-\frac{1}{p^{2 s+\alpha_{j}-\alpha_{k+i}}}\right)}{1-p^{\alpha_{k+i}-\alpha_{k+m}}} \times \prod_{p \mid N} \prod_{i=1}^{k} \prod_{j=1}^{k}\left(1-\frac{1}{p^{2 s+\alpha_{i}-\alpha_{k+j}}}\right) .
$$

In particular, if $\mathcal{L}(s)=L(s, \chi)$ with $\chi$ a Dirichlet character of conductor $N$, where the Riemann $\zeta$-function is the case $N=1$, then

$$
A_{1}\left(s ; \alpha_{1}, \alpha_{2}\right)=\prod_{p \mid N}\left(1-\frac{1}{p^{2 s+\alpha_{1}-\alpha_{2}}}\right)
$$

$$
A_{2}\left(s ; \alpha_{1}, \alpha_{2}, \alpha_{3}, \alpha_{4}\right)=\zeta\left(4 s+\alpha_{1}+\alpha_{2}-\alpha_{3}-\alpha_{4}\right)^{-1} \prod_{p \mid N} \prod_{i=1}^{2} \prod_{j=1}^{2}\left(1-\frac{1}{p^{2 s+\alpha_{i}-\alpha_{2+j}}}\right)
$$


and

$$
\begin{aligned}
& A_{3}\left(s ; \alpha_{1}, \ldots, \alpha_{6}\right) \\
& =\prod_{p \nmid N}\left(1-p^{-\sum_{1}^{3} \alpha_{i}-\alpha_{3+i}}\left(p^{\alpha_{1}}+p^{\alpha_{2}}+p^{\alpha_{3}}\right)\left(p^{-\alpha_{4}}+p^{-\alpha_{5}}+p^{-\alpha_{6}}\right) p^{-4 s}\right. \\
& +p^{-\sum_{1}^{3} \alpha_{i}-\alpha_{3+i}}\left(\left(p^{\alpha_{1}}+p^{\alpha_{2}}+p^{\alpha_{3}}\right)\left(p^{-\alpha_{1}}+p^{-\alpha_{2}}+p^{-\alpha_{3}}\right)\right. \\
& \left.+\left(p^{\alpha_{4}}+p^{\alpha_{5}}+p^{\alpha_{6}}\right)\left(p^{-\alpha_{4}}+p^{-\alpha_{5}}+p^{-\alpha_{6}}\right)-2\right) p^{-6 s} \\
& -p^{-\sum_{1}^{3} \alpha_{i}-\alpha_{3+i}}\left(p^{-\alpha_{1}}+p^{-\alpha_{2}}+p^{-\alpha_{3}}\right)\left(p^{\alpha_{4}}+p^{\alpha_{5}}+p^{\alpha_{6}}\right) p^{-8 s} \\
& \left.+p^{-2 \sum_{1}^{3} \alpha_{i}-\alpha_{3+i}} p^{-12 s}\right) \\
& \times \prod_{p \mid N} \prod_{i=1}^{3} \prod_{j=1}^{3}\left(1-\frac{1}{p^{2 s+\alpha_{i}-\alpha_{3+j}}}\right) \text {. }
\end{aligned}
$$

For $k \geq 3$ it is not possible to express $A_{k}$ as a finite product of $\zeta$-functions.

Proof of Lemma 2.6.1. Using $\mathcal{L}_{p}(x)=\left(1-\gamma_{p} x\right)^{-1}$ and setting

$$
q_{j}=\frac{\gamma_{p}}{p^{s+\alpha_{j}}} \quad \text { and } \quad q_{k+j}=\frac{\overline{\gamma_{p}}}{p^{s-\alpha_{k+j}}} \quad \text { for } j=1, \ldots, k,
$$

we have

$$
\begin{aligned}
B_{p}\left(s ; \alpha_{1}, \ldots, \alpha_{2 k}\right) & =\int_{0}^{1} \prod_{j=1}^{k}\left(1-e(\theta) q_{j}\right)^{-1}\left(1-e(-\theta) q_{k+j}\right)^{-1} d \theta \\
& =\frac{(-1)^{k}}{\prod_{j=1}^{k} q_{j}} \int_{0}^{1} e(k \theta) \prod_{j=1}^{k}\left(e(\theta)-1 / q_{j}\right)^{-1}\left(e(\theta)-q_{k+j}\right)^{-1} d \theta \\
& =\frac{(-1)^{k}}{\prod_{j=1}^{k} q_{j}} \frac{1}{2 \pi i} \oint z^{k-1} \prod_{j=1}^{k}\left(z-1 / q_{j}\right)^{-1}\left(z-q_{k+j}\right)^{-1} d z
\end{aligned}
$$

where the path of integration is around the unit circle. Since $\left|q_{j}\right|<1$, by the residue theorem we have a contribution from the poles at $q_{k+1}, \ldots, q_{2 k}$, giving

$$
\begin{aligned}
B_{p}\left(s ; \alpha_{1}, \ldots, \alpha_{2 k}\right) & =\frac{(-1)^{k}}{\prod_{j=1}^{k} q_{j}} \sum_{m=1}^{k} q_{k+m}^{k-1} \prod_{i=1}^{k}\left(q_{k+m}-q_{i}^{-1}\right)^{-1} \prod_{i \neq m}\left(q_{k+m}-q_{k+i}\right)^{-1} \\
& =\sum_{m=1}^{k} \prod_{i=1}^{k}\left(1-q_{i} q_{k+m}\right)^{-1} \prod_{i \neq m}\left(1-q_{k+i} q_{k+m}^{-1}\right)^{-1}
\end{aligned}
$$

Since

$$
\prod_{i, j=1}^{k}\left(1-q_{i} q_{k+j}\right) \prod_{i=1}^{k}\left(1-q_{i} q_{k+m}\right)^{-1}=\prod_{j \neq m} \prod_{i=1}^{k}\left(1-q_{i} q_{k+j}\right)
$$


factoring out

$$
\prod_{i, j=1}^{k}\left(1-q_{i} q_{k+j}\right)^{-1}
$$

we have

$$
B_{p}(s ; \alpha)=\left(\prod_{i, j=1}^{k}\left(1-q_{i} q_{k+j}\right)^{-1}\right) \sum_{m=1}^{k} \prod_{i \neq m} \frac{\prod_{j=1}^{k}\left(1-q_{j} q_{k+i}\right)}{1-q_{k+i} q_{k+m}^{-1}}
$$

Since

$$
q_{j} q_{k+i}=p^{-2 s-\alpha_{j}+\alpha_{k+i}} \quad \text { and } \quad q_{k+i} q_{k+m}^{-1}=p^{\alpha_{k+i}-\alpha_{k+m}},
$$

we obtain the formula in the Lemma.

Notice that the special case $N=1$, i.e. the Riemann $\zeta$ function, reads in Corollary 2.6.2.

$$
A_{k}\left(s ; \alpha_{1}, \ldots, \alpha_{2 k}\right)=\prod_{p} \sum_{m=1}^{k} \prod_{i \neq m} \frac{\prod_{j=1}^{k}\left(1-\frac{1}{p^{2 s+\alpha_{j}-\alpha_{k+i}}}\right)}{1-p^{\alpha_{k+i}-\alpha_{k+m}}} .
$$

Each local factor

$$
A_{p, k}(s ; \alpha)=\sum_{m=1}^{k} \prod_{i \neq m} \frac{\prod_{j=1}^{k}\left(1-\frac{1}{p^{2 s+\alpha_{j}-\alpha_{k+i}}}\right)}{1-p^{\alpha_{k+i}-\alpha_{k+m}}} .
$$

is actually a polynomial in $p^{-2 s}, p^{-\alpha_{j}}$ and $p^{\alpha_{k+j}}$, for $j=1, \ldots, k$. That this is so in $p^{-2 s}$ and $p^{-\alpha_{j}}$ is readily apparent from (2.6.16). The fact that it is also a polynomial in $p^{\alpha_{k+j}}$ follows from (2.4.2) and (2.4.3), from which

$$
A_{p, k}\left(s ; \alpha_{1}, \ldots, \alpha_{k}, \alpha_{k+1}, \ldots, \alpha_{2 k}\right)=A_{p, k}\left(s ;-\alpha_{k+1}, \ldots,-\alpha_{2 k},-\alpha_{1}, \ldots,-\alpha_{k}\right) .
$$

Setting $\beta_{1}=-\alpha_{k+1}, \ldots, \beta_{k}=-\alpha_{2 k}$, one has, from the above discussion, that $A_{p, k}$ is a polynomial in $p^{-\beta_{j}}$, i.e. in $p^{\alpha_{k+j}}$, for $j=1, \ldots, k$. Finally, use the fact that if an analytic function of several variables is of polynomial growth in each variable separately, then it must be a polynomial.

2.7. Recovering the leading order for moments of $\zeta$. Conjecture 1.5 .1 contains, as a special case, a conjecture for the leading order term for the moments of the Riemann zeta function. In this section we show that the leading order terms derived from Conjecture 1.5.1 agree with the leading order terms which have previously been conjectured by other methods.

As described in Section 1.3, it is conjectured that the mean values of the Riemann zeta function take the form

$$
\int_{0}^{T}\left|\zeta\left(\frac{1}{2}+i t\right)\right|^{2 k} d t=T \mathcal{P}_{k}(\log T)+O\left(T^{\frac{1}{2}+\varepsilon}\right),
$$

where $\mathcal{P}_{k}(\log T)$ is a polynomial in $\log T$ of degree $k^{2}$. Conrey and Ghosh conjectured that the coefficient of the $\log ^{k^{2}} T$ term is of the form $g_{k} a_{k} / k^{2}$ !, where $a_{k}$ is given by (1.3.2). 
Keating and Snaith used random matrix theory to conjecture that $g_{k}$ is given by (1.3.3). This leading order term $g_{k} a_{k} / k^{2}$ ! will be re-derived here, starting with Conjecture 1.5.1.

Conjecture 1.5 .1 implies

$$
\int_{0}^{T}\left|\zeta\left(\frac{1}{2}+i t\right)\right|^{2 k} d t=\int_{0}^{T} P_{k}\left(\log \frac{t}{2 \pi}\right) d t+O\left(T^{\frac{1}{2}+\varepsilon}\right)
$$

where $P_{k}$ is the polynomial of degree $k^{2}$ given by

$$
\begin{gathered}
P_{k}(x)=\frac{(-1)^{k}}{k !^{2}(2 \pi i)^{2 k}} \oint \cdots \oint A_{k}\left(z_{1}, \ldots, z_{2 k}\right) \prod_{i=1}^{k} \prod_{j=1}^{k} \zeta\left(1+z_{i}-z_{k+j}\right) \\
\times \frac{\Delta^{2}\left(z_{1}, \ldots, z_{2 k}\right)}{\prod_{j=1}^{2 k} z_{j}^{2 k}} e^{\frac{x}{2} \sum_{j=1}^{k} z_{j}-z_{k+j}} d z_{1} \cdots d z_{2 k} .
\end{gathered}
$$

Our goal is to show that the leading order term of $P_{k}(x)$ is $\left(g_{k} a_{k} / k^{2} !\right) x^{k^{2}}$.

Using the fact that $A_{k}$ is analytic in a neighborhood of $(0, \ldots, 0)$ and the $\zeta$-function has a simple pole at 1 with residue 1 , after a change of variables we have

$$
\begin{aligned}
& P_{k}(x)= \frac{(-1)^{k}}{k !^{2}(2 \pi i)^{2 k}} \oint \cdots \oint A_{k}\left(\frac{z_{1}}{x / 2}, \cdots, \frac{z_{2 k}}{x / 2}\right) \prod_{i=1}^{k} \prod_{j=1}^{k} \zeta\left(1+\frac{z_{i}-z_{k+j}}{x / 2}\right) \\
& \times \frac{\Delta^{2}\left(z_{1}, \ldots, z_{2 k}\right)}{\prod_{j=1}^{2 k} z_{j}^{2 k}} e^{\sum_{j=1}^{k} z_{j}-z_{k+j}} d z_{1} \cdots d z_{2 k} \\
&= \frac{(-1)^{k}}{k !^{2}} \frac{A_{k}(0, \ldots, 0)}{(2 \pi i)^{2 k}}\left(\frac{x}{2}\right)^{k^{2}}\left(1+O\left(x^{-1}\right)\right) \oint \cdots \oint \frac{\Delta^{2}\left(z_{1}, \ldots, z_{2 k}\right)}{\left(\prod_{i=1}^{k} \prod_{j=1}^{k}\left(z_{i}-z_{k+j}\right)\right) \prod_{j=1}^{2 k} z_{j}^{2 k}} \\
&= \frac{A_{k}(0, \ldots, 0)}{k !^{2} 2^{k^{2}}(2 \pi i)^{2 k}} x^{k^{2}}\left(1+O\left(x^{-1}\right)\right) \oint \cdots \oint \frac{\Delta\left(z_{1}, \ldots, z_{2 k}\right) \Delta\left(z_{1}, \ldots, z_{k}\right) \Delta\left(z_{k+1}, \ldots, z_{2 k}\right)}{\prod_{j=1}^{2 k} z_{j}^{2 k}} \\
& \quad \times e^{\sum_{j=1}^{k} z_{j}-z_{k+j}} d z_{1} \ldots, d z_{2 k} .
\end{aligned}
$$

Now we need only show that $A_{k}(0, \ldots 0)=a_{k}$ and the remaining factors give $g_{k} / k^{2}$.

From Conjecture 1.5.1.

$$
A_{k}(0, \ldots 0)=\prod_{p}\left(1-\frac{1}{p}\right)^{k^{2}} \int_{0}^{1}\left(1-\frac{e(\theta)}{p^{\frac{1}{2}}}\right)^{-k}\left(1-\frac{e(-\theta)}{p^{\frac{1}{2}}}\right)^{-k} d \theta .
$$

For a given $p$, we concentrate on the integral in the above expression, writing it as a contour integral around the unit circle:

$$
\left(-p^{\frac{1}{2}}\right)^{k} \frac{1}{2 \pi i} \oint \frac{z^{k-1}\left(z-p^{\frac{1}{2}}\right)^{-k}}{\left(z-p^{-\frac{1}{2}}\right)^{k}} d z .
$$

After expanding the two factors in the numerator around $z=p^{-\frac{1}{2}}$ and calculating the residue we are left with the sum

$$
\left(1-\frac{1}{p}\right)^{-2 k+1} \sum_{\ell=0}^{k-1}\left(\begin{array}{c}
k-1 \\
\ell
\end{array}\right)\left(\begin{array}{c}
2 k-\ell-2 \\
k-1
\end{array}\right) p^{-k+\ell+1}\left(1-\frac{1}{p}\right)^{\ell} .
$$


Next one can perform a binomial expansion of $\left(1-\frac{1}{p}\right)^{\ell}$ and gather like powers of $\frac{1}{p}$ to obtain

$$
\left(1-\frac{1}{p}\right)^{-2 k+1} \sum_{m=0}^{k-1}\left(\sum_{q=0}^{m}(-1)^{q}\left(\begin{array}{c}
k+q-m-1 \\
q
\end{array}\right)\left(\begin{array}{c}
k-1 \\
k+q-m-1
\end{array}\right)\left(\begin{array}{c}
k+m-q-1 \\
k-1
\end{array}\right)\right) p^{-m} .
$$

A simple manipulation of the binomial coefficients and replacing $q$ by $m-q$ gives

$$
\begin{gathered}
\sum_{q=0}^{m}(-1)^{q}\left(\begin{array}{c}
k+q-m-1 \\
q
\end{array}\right)\left(\begin{array}{c}
k-1 \\
k+q-m-1
\end{array}\right)\left(\begin{array}{c}
k+m-q-1 \\
k-1
\end{array}\right) \\
=\left(\begin{array}{c}
k-1 \\
m
\end{array}\right) \sum_{q=0}^{m}(-1)^{m-q}\left(\begin{array}{c}
m \\
q
\end{array}\right)\left(\begin{array}{c}
k+q-1 \\
q
\end{array}\right),
\end{gathered}
$$

and this final sum over $q$ is in fact just $\left(\begin{array}{c}k-1 \\ m\end{array}\right)$ (see, for example, [Rio]). Thus,

$$
A_{k}(0, \ldots, 0)=\prod_{p}\left(1-\frac{1}{p}\right)^{(k-1)^{2}} \sum_{m=0}^{k-1}\left(\begin{array}{c}
k-1 \\
m
\end{array}\right)^{2} p^{-m}
$$

and this is indeed equal to $a_{k}$ defined in (1.3.2).

Now we must identify the remaining terms as $g_{k} / k^{2}$ ! defined in (1.3.3), as $x \rightarrow \infty$. The method applied below as far as (2.7.14) follows closely that used for a similar purpose in [BH]. Expanding the determinants $\Delta\left(z_{1}, \ldots, z_{k}\right)=\operatorname{det}\left[z_{j}^{m-1}\right]_{j, m=1}^{k}$, we obtain

$$
\begin{aligned}
\lim _{x \rightarrow \infty} \frac{P_{k}(x)}{a_{k} x^{k^{2}}}= & \frac{1}{(k !)^{2} 2^{k^{2}}(2 \pi i)^{2 k}} \oint \cdots \oint e^{\sum_{j=1}^{k} z_{j}-z_{k+j}} \\
& \times\left(\sum_{S} \operatorname{sgn}(S) z_{1}^{S_{0}} z_{2}^{S_{1}} \cdots z_{k}^{S_{k-1}} z_{k+1}^{S_{k}} \cdots z_{2 k}^{S_{2 k-1}}\right)\left(\sum_{Q} \operatorname{sgn}(Q) z_{1}^{Q_{0}} \cdots z_{k}^{Q_{k-1}}\right) \\
& \times\left(\sum_{R} \operatorname{sgn}(R) z_{k+1}^{R_{0}} \cdots z_{2 k}^{R_{k-1}}\right) z_{1}^{-2 k} \cdots z_{2 k}^{-2 k} d z_{1} \cdots d z_{2 k} .
\end{aligned}
$$

Here $Q$ and $R$ are permutations of $\{0,1, \ldots, k-1\}$ and $S$ is a permutation of $\{0,1, \ldots, 2 k-1\}$.

Since the integrand is symmetric amongst $z_{1}, \ldots, z_{k}$ and also amongst $z_{k+1}, \ldots, z_{2 k}$, in each term of the sum over $Q$ we permute the variables $z_{1}, \ldots, z_{k}$ so that $z_{j}$ appears with the exponent $j-1$, for $j=1, \ldots, k$. In the sum over $S$ the effect is to redefine the permutations, and the additional sign involved with this exactly cancels $\operatorname{sgn}(Q)$. We do the same with the sum over $R$, and as a result we are left with $k !^{2}$ copies of the sum over the permutation $S$ :

$$
\begin{aligned}
\lim _{x \rightarrow \infty} \frac{P_{k}(x)}{a_{k} x^{k^{2}}}= & \frac{1}{2^{k^{2}}(2 \pi i)^{2 k}} \oint \cdots \oint e^{\sum_{j=1}^{k} z_{j}-z_{k+j}} \\
& \times \sum_{S} \operatorname{sgn}(S) z_{1}^{-\left(2 k-S_{0}\right)} z_{2}^{-\left(2 k-S_{1}-1\right)} \cdots z_{k}^{-\left(2 k-S_{k-1}-(k-1)\right)} \\
& \times z_{k+1}^{-\left(2 k-S_{k}\right)} z_{k+2}^{-\left(2 k-S_{k+1}-1\right)} \cdots z_{2 k}^{-\left(2 k-S_{2 k-1}-(k-1)\right)} d z_{1} \cdots d z_{2 k} .
\end{aligned}
$$


Since

$$
\frac{1}{\Gamma(z)}=\frac{1}{2 \pi i} \int_{C}(-t)^{-z} e^{-t}(-d t)
$$

where the path of integration $C$ starts at $+\infty$ on the real axis, circles the origin in the counterclockwise direction and returns to the starting point, we can rewrite (2.7.12) as

$$
\begin{aligned}
& \lim _{x \rightarrow \infty} \frac{P_{k}(x)}{a_{k} x^{k^{2}}}=\frac{(-1)^{k}}{2^{k^{2}}} \sum_{S} \operatorname{sgn}(S)\left(\Gamma\left(2 k-S_{0}\right) \Gamma\left(2 k-S_{1}-1\right) \cdots \Gamma\left(2 k-S_{k-1}-(k-1)\right)\right. \\
& \times(-1)^{S_{k}} \Gamma\left(2 k-S_{k}\right)(-1)^{S_{k+1}+1} \Gamma\left(2 k-S_{k+1}-1\right) \cdots \\
& \left.\times(-1)^{S_{2 k-1}+k-1} \Gamma\left(2 k-S_{2 k-1}-(k-1)\right)\right)^{-1} \\
& =\frac{(-1)^{k}}{2^{k^{2}}}\left|\begin{array}{cccccccc}
\frac{1}{\Gamma(2 k)} & \frac{1}{\Gamma(2 k-1)} & \cdots & \frac{1}{\Gamma(k+1)} & \frac{1}{\Gamma(2 k)} & \frac{-1}{\Gamma(2 k-1)} & \cdots & \frac{(-1)^{k-1}}{\Gamma(k+1)} \\
\frac{1}{\Gamma(2 k-1)} & \frac{1}{\Gamma(2 k-2)} & \cdots & \frac{1}{\Gamma(k)} & \frac{-1}{\Gamma(2 k-1)} & \frac{1}{\Gamma(2 k-2)} & \cdots & \frac{(-1)^{k}}{\Gamma(k)} \\
\vdots & \vdots & \ddots & \vdots & \vdots & \vdots & \ddots & \vdots \\
\frac{1}{\Gamma(1)} & \frac{1}{\Gamma(0)} & \cdots & \frac{1}{\Gamma(2-k)} & \frac{-1}{\Gamma(1)} & \frac{1}{\Gamma(0)} & \cdots & \frac{(-1)^{3 k-2}}{\Gamma(2-k)}
\end{array}\right| \\
& =\frac{(-1)^{k}}{2^{k^{2}}}\left(\prod_{\ell=0}^{k-1} \frac{\ell !}{(k+\ell) !}\right)
\end{aligned}
$$

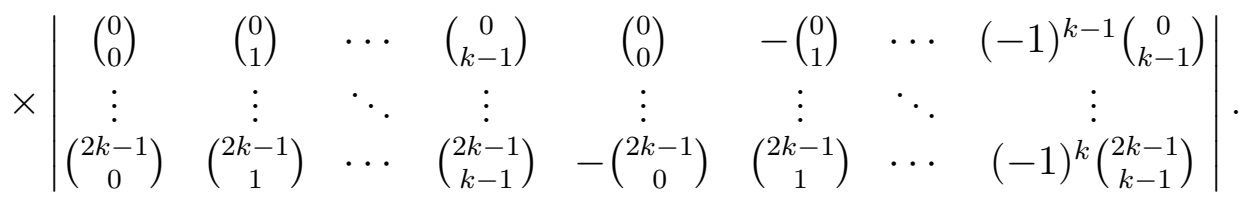

The above is a $2 k \times 2 k$ determinant, the first $k$ columns of which are identical to the first $k$ columns of the matrix

$$
\left(\begin{array}{cccc}
\left(\begin{array}{l}
0 \\
0
\end{array}\right) & \left(\begin{array}{l}
0 \\
1
\end{array}\right) & \cdots & \left(\begin{array}{c}
0 \\
2 k-1
\end{array}\right) \\
\vdots & \vdots & \ddots & \vdots \\
\left(\begin{array}{c}
2 k-1 \\
0
\end{array}\right) & \left(\begin{array}{c}
2 k-1 \\
1
\end{array}\right) & \cdots & \left(\begin{array}{l}
2 k-1 \\
2 k-1
\end{array}\right)
\end{array}\right)
$$

The matrix (2.7.15) is lower triangular and so can easily be seen to have determinant equal to one. It is also the inverse of

$$
\left(\begin{array}{cccc}
\left(\begin{array}{l}
0 \\
0
\end{array}\right) & -\left(\begin{array}{l}
0 \\
1
\end{array}\right) & \cdots & -\left(\begin{array}{c}
0 \\
2 k-1
\end{array}\right) \\
-\left(\begin{array}{c}
1 \\
0
\end{array}\right) & \left(\begin{array}{c}
1 \\
1
\end{array}\right) & \cdots & \left(\begin{array}{c}
1 \\
2 k-1
\end{array}\right) \\
\vdots & \vdots & \ddots & \vdots \\
-\left(\begin{array}{c}
2 k-1 \\
0
\end{array}\right) & \left(\begin{array}{c}
2 k-1 \\
1
\end{array}\right) & \cdots & \left(\begin{array}{c}
2 k-1 \\
2 k-1
\end{array}\right)
\end{array}\right)
$$

It so happens that matrix (2.7.16) has its $k$ first columns identical to columns $k+1$ through $2 k$ of the matrix in expression (2.7.14). Therefore we can multiply expression (2.7.14) by the determinant of (2.7.15) (which is equal to one) and this simplifies the final $k$ columns of the 
resulting determinant significantly:

$$
\begin{aligned}
& \lim _{x \rightarrow \infty} \frac{P_{k}(x)}{a_{k} x^{k^{2}}} \\
& =\frac{(-1)^{k}}{2^{k^{2}}}\left(\prod_{\ell=0}^{k-1} \frac{\ell !}{(k+\ell) !}\right)
\end{aligned}
$$

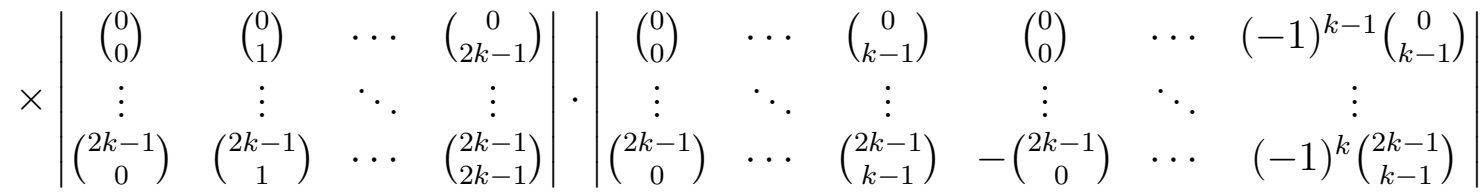

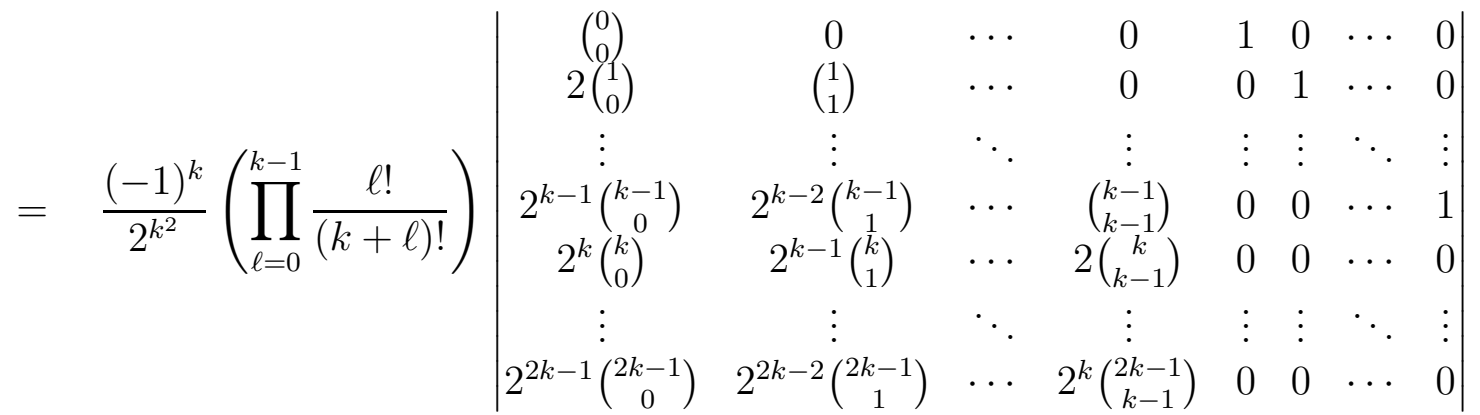

$$
\begin{aligned}
& =\frac{(-1)^{k(k-1) / 2}}{2^{k^{2}}}\left(\prod_{\ell=1}^{k-1} \frac{\ell !}{(k+\ell) !}\right)\left|\begin{array}{cccc}
2^{2 k-1}\left(\begin{array}{c}
2 k-1 \\
0
\end{array}\right) & 2^{2 k-2}\left(\begin{array}{c}
2 k-1 \\
1
\end{array}\right) & \cdots & 2^{k}\left(\begin{array}{c}
2 k-1 \\
k-1
\end{array}\right) \\
\vdots & \vdots & \ddots & \vdots \\
2^{k}\left(\begin{array}{c}
k \\
0
\end{array}\right) & 2^{k-1}\left(\begin{array}{c}
k \\
1
\end{array}\right) & \cdots & 2\left(\begin{array}{c}
k \\
k-1
\end{array}\right)
\end{array}\right|
\end{aligned}
$$

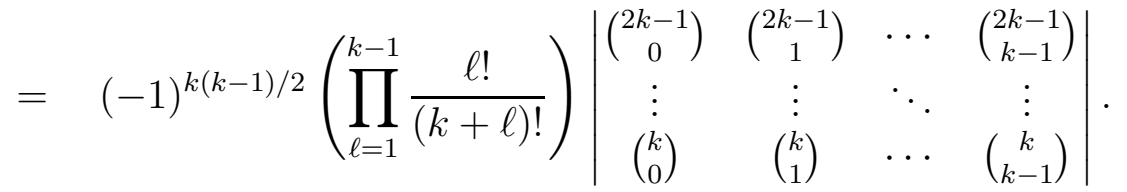

The matrix above can be decomposed as

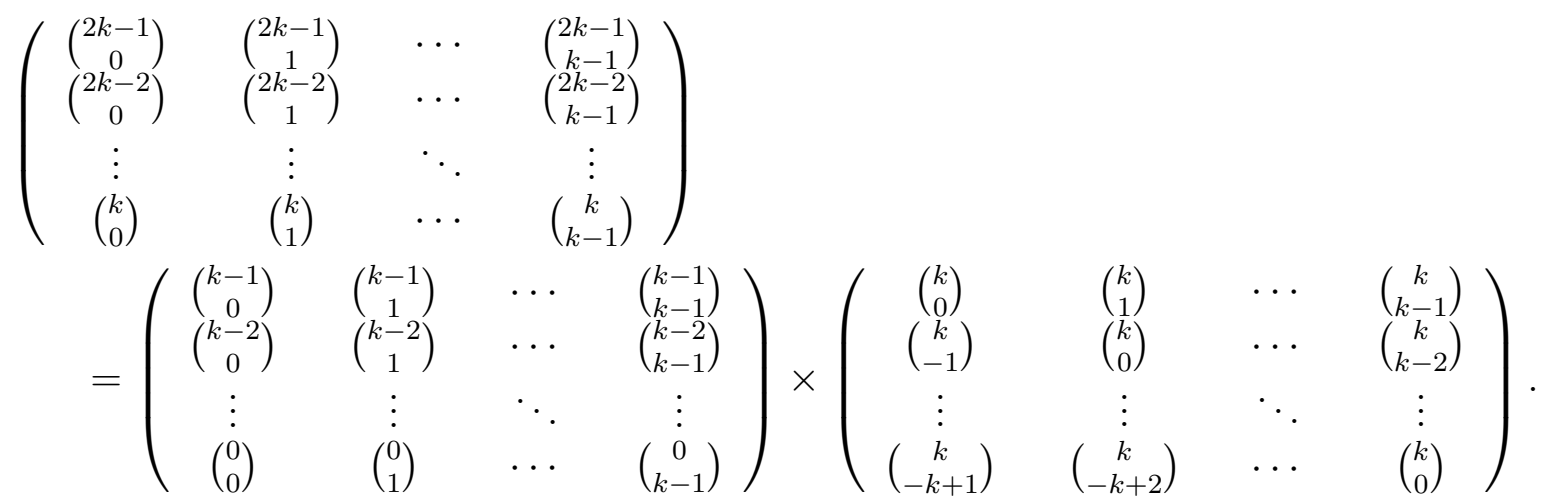

The first matrix on the right side is zero in the lower right triangle, and the second matrix on the right side is upper triangular. Thus we read that the determinant of the matrix on the left hand side is $(-1)^{k(k-1) / 2}$. Therefore,

$$
\lim _{x \rightarrow \infty} \frac{P_{k}(x)}{a_{k} x^{k^{2}}}=\prod_{\ell=1}^{k-1} \frac{\ell !}{(k+\ell) !},
$$

and this is $g_{k} / k^{2}$ ! from (1.3.3), as required. 
A similar method applies to the orthogonal and symplectic cases.

\section{FAMilies OF CHARACTERS AND FAMILIES OF L-FUNCTIONS}

We will describe a particular kind of "family" of primitive $L$-functions based on the idea of twisting a single $L$-function by a family of "characters." In the next section we provide a general recipe for conjecturing the critical mean value of products of $L$-functions averaged over a family and we demonstrate the recipe in several examples.

Note that we use "character" somewhat more generally than is usually covered by that term.

3.1. Families of primitive characters. We describe sets of arithmetic functions which we call "families of characters."

Let $\mathcal{F}=\{f\}$ be a collection of arithmetic functions $f(n)$, and assume that for each $f \in \mathcal{F}$ the associated $L$-function $L_{f}(s)=\sum f(n) n^{-s}$ is a primitive $L$-function with functional equation $L_{f}(s)=\varepsilon_{f} X_{f}(s) \bar{L}_{f}(1-s)$ and an Euler product of the form

$$
L_{f}(s)=\sum_{n=1}^{\infty} \frac{f(n)}{n^{s}}=\prod_{p} \prod_{j=1}^{v}\left(1-\beta_{p, j} / p^{s}\right)^{-1} .
$$

The quantity

$$
c(f)=\left|\left(\varepsilon_{f} X_{f}\right)^{\prime}\left(\frac{1}{2}\right)\right|
$$

is called the $\log$ conductor of $f$.

Note that if $f=\chi$, a primitive Dirichlet character of conductor $q$, then the log conductor is

$$
c(\chi)=\left\{\begin{array}{lll}
\log q-\log \pi+\frac{\Gamma^{\prime}}{\Gamma}\left(\frac{1}{4}\right) & \chi & \text { even } \\
\log q-\log \pi+\frac{\Gamma^{\prime}}{\Gamma}\left(\frac{3}{4}\right) & \chi & \text { odd. }
\end{array}\right.
$$

If $f(n)=n^{-i t}$ then the $\log$ conductor is $c\left(n^{-i t}\right)=\log \frac{t}{2 \pi}+O\left(t^{-1}\right)$. Generally the log conductor $c(f)$ scales as the log of the "usual" conductor of $f$.

In the case that $\mathcal{F}$ is finite, we require that the data $Q, w_{j}, \mu_{j}$ in the functional equation (11.1.2) is the same for all $f \in \mathcal{F}$. In particular, the conductor $c(f)$ is the same for all $f \in \mathcal{F}$.

In the case that $\mathcal{F}$ is infinite, we require that the data $Q, w_{j}, \mu_{j}$ in the functional equation (1.1.2) are monotonic functions of the conductor $c(f)$. Furthermore, we define the counting function $M(T)=\#\{f: c(f) \leq T\}$ and require that $M(\log (T))=F\left(T^{A}, \log T\right)+O\left(T^{\frac{A}{2}+\epsilon}\right)$ for all $\epsilon>0$, where $A>0$ and $F(\cdot, \cdot)$ is a polynomial.

If $G$ is a function on $\mathcal{F}$, then we define the expected value of $G$ by

$$
\langle G(f)\rangle=\lim _{T \rightarrow \infty} M(T)^{-1} \sum_{\substack{f \in \mathcal{F} \\ c(f)<T}} G(f),
$$

assuming the limit exists. In the case of a continuous family, the sum is an integral.

We require that if $m_{1}, \ldots, m_{k}$ are integers then the expected value

$$
\delta_{\ell}\left(m_{1}, \ldots, m_{k}\right)=\left\langle f\left(m_{1}\right) \ldots f\left(m_{\ell}\right) \overline{f\left(m_{\ell+1}\right) \ldots f\left(m_{k}\right)}\right\rangle
$$


exists and is multiplicative. That is, if $\left(m_{1} m_{2} \ldots m_{k}, n_{1} n_{2} \ldots n_{k}\right)=1$, then

$$
\delta_{\ell}\left(m_{1} n_{1}, m_{2} n_{2}, \ldots m_{k} n_{k}\right)=\delta_{\ell}\left(m_{1}, \ldots, m_{k}\right) \delta_{\ell}\left(n_{1}, \ldots n_{k}\right) .
$$

We sometimes refer to $\delta$ as the "orthogonality relation" of the family.

The practical use of being multiplicative is that a multiple Dirichlet series with $\delta_{\ell}$ coefficients factors has an Euler product:

$$
\sum_{m_{1}, \ldots, m_{\ell}} \frac{\delta_{\ell}\left(m_{1}, \ldots, m_{\ell}\right)}{m_{1}^{s_{1}} \cdots m_{\ell}^{s_{\ell}}}=\prod_{p} \sum_{e_{1}, \ldots, e_{\ell}} \frac{\delta_{\ell}\left(p^{e_{1}}, \ldots, p^{e_{\ell}}\right)}{p^{e_{1} s_{1}+\cdots+e_{\ell} s_{\ell}}} .
$$

We will use the above relation in our calculations.

To summarize, a family of characters $\mathcal{F}=\{f\}$ is a collection of arithmetic functions, each of which are the coefficients of a particular kind of $L$-function. The characters are partially ordered by conductor $c(f)$, and the expected values $\delta_{\ell}\left(m_{1}, \ldots, m_{k}\right)$ are multiplicative functions.

The following are examples of families of characters, two of which are finite and two are infinite. The term "finite family" is somewhat misleading, because those families depend on a parameter, and the size of the family grows with the parameter.

\section{The family of $t$-twists.}

$$
\mathcal{F}_{\mathrm{t}}=\left\{f_{t}(n)=n^{-i t}, 0<t<T\right\} .
$$

We have

$$
\frac{1}{T} \int_{0}^{T}(m / n)^{i t} d t= \begin{cases}1 & n=m \\ \frac{(m / n)^{i T}-1}{T \log (m / n)} & \text { otherwise, }\end{cases}
$$

leading to the expected values

$$
\left\langle f_{t}(n) \overline{f_{t}(m)}\right\rangle=\left\langle n^{-i t} m^{i t}\right\rangle=\left\langle(m / n)^{i t}\right\rangle= \begin{cases}1 & n=m \\ 0 & \text { otherwise. }\end{cases}
$$

Therefore the orthogonality relation is

$$
\delta_{\ell}\left(n_{1}, \ldots, n_{k}\right)=\delta\left(n_{1} \cdots n_{\ell}=n_{\ell+1} \cdots n_{k}\right) .
$$

2. The family of primitive Dirichlet characters. For each positive integer $q$ we set

$$
\mathcal{F}_{\text {ch }}(q)=\left\{f_{\chi}(n)=\chi(n): \chi \text { is a primitive Dirichlet character } \bmod q\right\} .
$$

We have

$$
\frac{1}{q^{*}} \sum_{\chi \bmod q}^{*} \chi(n) \overline{\chi(m)}= \begin{cases}1 & n \equiv m \bmod q \text { and }(m n, q)=1 \\ 0 & \text { otherwise, }\end{cases}
$$

where the sum is over the primitive characters $\bmod q$, and $q^{*}$ is the number terms in the sum. This leads to the expected values

$$
\left\langle f_{\chi}(n) \overline{f_{\chi}(m)}\right\rangle=\langle\chi(n) \overline{\chi(m)}\rangle= \begin{cases}1 & n=m \text { and }(m n, q)=1 \\ 0 & \text { otherwise }\end{cases}
$$

Since $\chi\left(m_{1}\right) \chi\left(m_{2}\right)=\chi\left(m_{1} m_{2}\right)$ we obtain

$$
\delta_{\ell}\left(m_{1}, \ldots, m_{k}\right)=\delta\left(m_{1} \cdots m_{\ell}=m_{\ell+1} \cdots m_{k} \quad \text { and } \quad\left(m_{1} \cdots m_{k}, q\right)=1\right) .
$$


Note that the condition in the definition of $\delta_{\ell}$ is not $m_{1} \cdots m_{\ell} \equiv m_{\ell+1} \cdots m_{k}$ mod $q$. We are computing the expected value as a function of $q$, so one should think of the $m_{j}$ as fixed and $q \rightarrow \infty$. The only way to have $m_{1} \cdots m_{\ell} \equiv m_{\ell+1} \cdots m_{k} \bmod q$ for sufficiently large $q$ is to have actual equality.

Note that by our definition, $\mathcal{F}_{\mathrm{ch}}(q)$ is not a family, but it is the union of two families consisting of the even characters and the odd characters separately.

\section{The family of real primitive Dirichlet characters.}

$$
\mathcal{F}_{\mathrm{d}}=\left\{f_{d}(n)=\chi_{d}(n): \chi_{d} \text { is a primitive real character } \bmod d,|d|<X\right\},
$$

where $d$ runs over fundamental discriminants. We have expected values

$$
\left\langle f_{d}(n) f_{d}(m)\right\rangle=\left\langle\chi_{d}(n) \chi_{d}(m)\right\rangle=\left\langle\chi_{d}(n m)\right\rangle=\left\{\begin{array}{ll}
\prod_{p \mid n m}\left(1+\frac{1}{p}\right)^{-1} & n m=\square \\
0 & \text { otherwise. }
\end{array} .\right.
$$

The calculation in the case $n m=\square$ is nontrivial and was first done by Jutila[J]. (If one were summing over all $d$ then the expected value when $n m=\square$ would be $\varphi(n m) / n m)$.

In practice one encounters more restricted families, so we let $\mathcal{F}_{\mathrm{d}}(+)=\left\{f_{d}: d>0\right\}$ and $\mathcal{F}_{\mathrm{d}}(-)=\left\{f_{d}: d<0\right\}$, and also

$$
\mathcal{F}_{\mathrm{d}}(a, N, \pm)=\left\{f_{d} \in \mathcal{F}_{\mathrm{d}}( \pm): d \equiv a \bmod N\right\}
$$

For the family $\mathcal{F}_{\mathrm{d}}(a, N, \pm)$, evaluating the expected value of $\chi_{d}(n)$ can be tricky, so we provide some useful asymptotics.

Below we restrict ourselves to $0<d<X$, but the same asymptotics hold if one restricts to $-X<d<0$.

Theorem 3.1.1. Let $Q=\operatorname{gcd}(a, N)$ not be divisible by the square of an odd prime. Then

$$
\sum_{\substack{0<d<X \\ d \equiv a \bmod N}}^{*} 1 \sim \frac{1}{\phi(4 N / Q)} \frac{X}{Q} \frac{6}{\pi^{2}} h_{2}(a, N) \prod_{p \mid 2 N} \frac{p}{p+1} .
$$

Next, assume further that either $N$ is odd or divisible by at least 8 (this condition is related to the fact that $\chi_{d}(2)$ is periodic mod 8), and say $n=g \square$, with $(\square, N)=1$, and with all prime factors of $g$ being prime factors of $N$. Then

$$
\sum_{\substack{0<d<X \\ d \equiv a \bmod N}}^{*} \chi_{d}(n) \sim \chi_{a}(g) \phi(\square) \frac{1}{\phi(4 N \square / Q)} \frac{X}{Q} \frac{6}{\pi^{2}} h_{2}(a, N) \prod_{p \mid 2 N \square} \frac{p}{p+1} .
$$

Here $h_{2}(a, N)$ is determined according to Table 3.1.1. Consequently, for the family $\mathcal{F}_{d}(a, N, \pm)$,

$$
\left\langle\chi_{d}(n)\right\rangle= \begin{cases}\chi_{a}(g) \prod_{p \mid \square}\left(1+\frac{1}{p}\right)^{-1} & n=g \square \\ 0 & \text { otherwise }\end{cases}
$$

Proof. We first outline the proof for (3.1.19). One can count odd fundamental discriminants $|d|<X$ by using the Dirichlet series

$$
\sum_{d \text { odd }}^{*} \frac{1}{|d|^{s}}=\prod_{p \text { odd }}\left(1+\frac{1}{p^{s}}\right)=\frac{\zeta(s)}{\zeta(2 s)}\left(1+\frac{1}{2^{s}}\right)^{-1}
$$




\begin{tabular}{|c|c|c|}
$\beta$ & $a$ & $h_{2}(a, N)$ \\
\hline \hline 0 & $a \in \mathbb{Z}$ & $3 / 2$ \\
\hline 1 & $a \equiv 0 \bmod 2$ & 1 \\
& $a \equiv 1 \bmod 2$ & 2 \\
\hline 2 & $a \equiv 0 \bmod 4$ & 2 \\
& $a \equiv 1 \bmod 4$ & 4 \\
& $a \equiv 2,3 \bmod 4$ & 0 \\
\hline 3 & $a \equiv 0,4 \bmod 8$ & 2 \\
& $a \equiv 1,5 \bmod 8$ & 4 \\
& $a \equiv 2,3,6,7 \bmod 8$ & 0 \\
\hline$\geq 4$ & $a \equiv 1,5,8,9,12,13 \bmod 16$ & 4 \\
& otherwise & 0 \\
\hline
\end{tabular}

TABLE 3.1.1. The function $h_{2}(a, N)$ that appears in Theorem 3.1.1, where $N=2^{\beta} N_{0}$, with $N_{0}$ odd.

As in the proof of the prime number theorem, the main contribution comes from the pole at $s=1$, and one has

$$
\sum_{\substack{|d|<X \\ d \text { odd }}}^{*} 1 \sim \frac{4}{\pi^{2}} X
$$

Next, assume that $N$ is odd and $(a, N)=1$. To count odd fundamental discriminants in arithmetic progression, $d \equiv a \bmod N$, one imitates Dirichlet's theorem for primes in arithmetic progression, looking at linear combinations involving Dirichlet characters mod $N$ of

$$
\sum_{d \text { odd }}^{*} \frac{\chi(d)}{|d|^{s}}=\prod_{p \text { odd }}\left(1+\frac{\chi(p)}{p^{s}}\right) .
$$

If one wishes to further specify $d>0$ or $d<0$, one can restrict to $|d| \equiv 1 \bmod 4$ or $|d| \equiv 3 \bmod 4$ respectively, with $\chi$ ranging over Dirichlet character mod $4 N$. The main contribution comes from the trivial character whose corresponding Dirichlet series is

$$
\sum_{d \text { odd }}^{*} \frac{\chi_{0}(d)}{|d|^{s}}=\prod_{\substack{p \text { odd } \\ p \nmid N}}\left(1+\frac{1}{p^{s}}\right)=\frac{\zeta(s)}{\zeta(2 s)} \prod_{p \mid 2 N}\left(1+\frac{1}{p^{s}}\right)^{-1},
$$

and whose main pole is at $s=1$. Therefore, for $N$ odd and $(a, N)=1$, we have

$$
\sum_{\substack{0<d<X \\ d \equiv a \text { mod } N \\ d \text { odd }}}^{*} 1 \sim \frac{1}{\phi(4 N)} X \frac{6}{\pi^{2}} \prod_{p \mid 2 N} \frac{p}{p+1},
$$

with the same result for $-X<d<0$.

Next, for $N$ odd and $(a, N)=Q>1$, one can write, for $d \equiv a \bmod N, d=d_{1} Q$. Apply the above method to $d_{1}$ with $0<d_{1}<X / Q, d_{1} \equiv(a / Q) \bmod (N / Q), d_{1}$ odd, and, because $d$ is squarefree, the extra condition that $\left(d_{1}, Q\right)=1$. Because of this last condition, the Euler product that we need to take in (3.1.25) is not just over odd $p \nmid(N / Q)$ but also $p \nmid Q$, i.e. it 
is still

$$
\prod_{\substack{p \text { odd } \\ p \nmid N}}\left(1+\frac{1}{p^{s}}\right)
$$

Hence, if $(a, N)=Q$,

$$
\sum_{\substack{0<d<X \\ d \equiv a \text { mod } N \\ d \text { odd }}}^{*} 1 \sim \frac{1}{\phi(4 N / Q)} \frac{X}{Q} \frac{6}{\pi^{2}} \prod_{p \mid 2 N} \frac{p}{p+1}
$$

and the same result for $-X<d<0$.

Finally, we wish to take into account even $d$. The set of even fundamental discriminants consists of -4 and \pm 8 times the odd fundamental discriminants.

Again, assume $N$ is odd. One can count discriminants, $d \equiv a \bmod N$, lying in the interval $(0, X)$ by counting odd discriminants lying in $(0, X)$, together with odd discriminants in $(-X / 4,0),(0, X / 8)$ and $(-X / 8,0)$. Overall, this gives the same asymptotics as before, but with an extra factor of $(1+1 / 4+2 / 8)=3 / 2$. This accounts for line 1 in Table 3.1.1. The other lines in the table can be obtained by similar considerations.

We now apply (3.1.19) to obtain (3.1.20) and (3.1.21). Consider

$$
\frac{\sum_{\substack{0<d<X \\ d \equiv a \bmod N}}^{*} \chi_{d}(n)}{\sum_{\substack{0<d<X \\ d \equiv a \bmod N}}^{*} 1}
$$

(the following analysis also holds for $-X<d<0$ ).

Write $N=N_{1}^{r_{1}} \cdot \ldots \cdot N_{m}^{r_{m}}$, the prime factorization of $N$, and let $g=N_{1}^{u_{1}} \cdot \ldots \cdot N_{m}^{u_{m}}$. Then

$$
\chi_{d}(n)=\chi_{d}\left(N_{1}\right)^{u_{1}} \ldots \chi_{d}\left(N_{m}\right)^{u_{m}} \chi_{d}(\square) .
$$

Now, if $N_{i}$ is an odd prime, $\chi_{d}\left(N_{i}\right)=\chi_{a}\left(N_{i}\right)$, since $d \equiv a \bmod N$, and so $d \equiv a \bmod N_{i}$. If $N_{i}=2$ we need to be careful because $\chi_{d}(2)$ is periodic mod 8 . Now we are assuming that if $N$ is even it be a least divisible by 8 , i.e. that $d \equiv a \bmod 8$, and thus that $\chi_{d}(2)=\chi_{a}(2)$.

Therefore

$$
\chi_{d}(n)=\chi_{a}(g) \chi_{d}(\square)= \begin{cases}\chi_{a}(g) & \text { if }(d, \square)=1 \\ 0 & \text { otherwise }\end{cases}
$$

and one gets that (3.1.29) equals

$$
\chi_{a}(g) \frac{\sum_{\substack{0<d<X \\ d \equiv a \text { mod } N \\(d, \square)=1}}^{*} 1}{\sum_{\substack{0<d<X \\ d \equiv a \bmod N}}^{*} 1} .
$$


Since $(\square, N)=1$, the sum in the numerator can be split into sums $d \bmod N \square$. Naively, one expects to have $\phi(\square)$ sums, one for each residue class $(d, \square)=1$. However, if $\square$ is even, then only half of these residue classes, namely those that have $d \equiv 1 \bmod 4$, contain fundamental discriminants, so one only gets $\phi(\square) / 2$ sums. We thus consider the case that $\square$ is odd separately from the case that it is even. Both cases end up giving the same answer.

Assume that $\square$ is odd. To apply our formula (3.1.19) to each of the $\phi(\square)$ residue classes $\bmod N \square$, one needs to compute the various components that go into the formula.

Given $d \equiv a \bmod N$ and $d \equiv b \bmod \square$, one has via the chinese remainder theorem $d \equiv \tilde{a} \bmod N \square$. Now, $Q=(a, N)=(d, N)$, and $(d, \square)=1$, so $(\tilde{a}, N \square)=(d, N \square)=Q$.

One also needs to evaluate $h_{2}(\tilde{a}, N \square)$. Let $N=2^{\beta} N_{0}$, with $N_{0}$ odd. Now $\square$ is odd, and so $h_{2}(\tilde{a}, N \square)$ only depends on $\tilde{a} \bmod 2^{\beta}$, but this is determined by $a \bmod N$. So $h_{2}(\tilde{a}, N \square)=$ $h_{2}(a, N)$. Therefore, the numerator of (3.1.32) is asymptotically

$$
\chi_{a}(g) \phi(\square) \frac{1}{\phi(4 N \square / Q)} \frac{X}{Q} \frac{6}{\pi^{2}} h_{2}(a, N) \prod_{p \mid 2 N \square} \frac{p}{p+1} .
$$

Canceling factors appearing in the asymptotics (3.1.19) of the denominator of (3.1.32) we get

$$
\chi_{a}(g) \prod_{p \mid \square} \frac{p}{p+1}
$$

If $\square$ is even, write $\square=2^{\lambda} \square_{0}$, with $\lambda \geq 2$, and $\square_{0}$ odd. Now, $(d, \square)=1$, so $d$ is odd. In all cases, according to Table 3.1.1, $h_{2}(\tilde{a}, N \square)$ is therefore 4. Furthermore, as in the odd case, $(\tilde{a}, N \square)=Q$.

Hence, one gets asymptotically for the numerator of (3.1.32)

$$
\chi_{a}(g) \frac{\phi(\square)}{2} \frac{1}{\phi(4 N \square / Q)} \frac{X}{Q} \frac{6}{\pi^{2}} 4 \prod_{p \mid 2 N \square} \frac{p}{p+1} .
$$

Since $\square$ is even, $N$ is odd. Hence $h_{2}(a, N)=3 / 2$, and the denominator of (3.1.32) is asymptotically

$$
\frac{1}{\phi(4 N / Q)} \frac{X}{Q} \frac{6}{\pi^{2}} \frac{3}{2} \prod_{p \mid 2 N} \frac{p}{p+1} .
$$

Canceling numerator and denominator, taking special care for powers of 2 appearing in $\square$, we get

$$
\chi_{a}(g) \frac{2}{3} \prod_{p \mid \square_{0}} \frac{p}{p+1}=\chi_{a}(g) \prod_{p \mid \square} \frac{p}{p+1}
$$

\section{The family of coefficients of holomorphic newforms.}

$$
\mathcal{F}_{\text {mod }}(k, q)=\left\{f(n)=\lambda_{f}(n): \sum n^{(k-1) / 2} \lambda_{f}(n) \in H_{k}(q)\right\},
$$


where $H_{k}(q)$ the set of newforms in $S_{k}\left(\Gamma_{0}(q)\right)$. A good reference for these functions is Iwaniec[Iw1]. In this family the parameter tending to infinity can be either $k$, or $q$, or some combination.

The orthogonality relation here is somewhat subtle, and in fact there are two natural ways to average over these characters. In both cases the starting point is the Hecke relation

$$
\lambda_{f}(m) \lambda_{f}(n)=\sum_{\substack{d|m, d| n \\(d, q)=1}} \lambda_{f}\left(m n / d^{2}\right),
$$

which imply that any product

$$
\lambda_{f}\left(m_{1}\right) \cdots \lambda_{f}\left(m_{k}\right)
$$

can be expressed as a linear combination

$$
\sum_{j \geq 1} b_{j} \lambda_{f}(j)
$$

for some integers $b_{j}$, and in fact only for $j$ a prime power. Thus, we need only determine the expected value of $\lambda_{f}\left(p^{j}\right)$.

If one averages over $H_{k}(q)$ in the most straightforward way, then for $p \nmid q$,

$$
\left\langle\lambda_{f}\left(p^{j}\right)\right\rangle=\left\{\begin{array}{lll}
p^{-j / 2} & j & \text { even } \\
0 & j & \text { odd }
\end{array}\right.
$$

and more generally, if $(n, q)=1$,

$$
\left\langle\lambda_{f}(n)\right\rangle= \begin{cases}n^{-1 / 2} & n=\square \\ 0 & \text { otherwise }\end{cases}
$$

This follows from the Selberg trace formula. However, if one averages with respect to a weighting by the Petersson norm:

$$
\sum_{f \in H_{k}(q)}^{\mathrm{h}} *=\sum_{f \in H_{k}(q)} * /\langle f, f\rangle,
$$

then

$$
\left\langle\lambda_{f}\left(p^{j}\right)\right\rangle= \begin{cases}1 & j=0 \\ 0 & \text { otherwise }\end{cases}
$$

and more generally, if $(n, q)=1$,

$$
\left\langle\lambda_{f}(n)\right\rangle= \begin{cases}1 & n=1 \\ 0 & \text { otherwise. }\end{cases}
$$

This follows from the Petersson formula (see [Iw1]), if $(m n, q)=1$,

$$
\sum_{f \in H_{k}(q)}^{\mathrm{h}} \lambda_{f}(m) \lambda_{f}(n)=\delta(m, n)+2 \pi i^{k} \sum_{c=1}^{\infty} \frac{S(m, n ; c q) J_{k-1}(4 \pi \sqrt{m n} / c q)}{c q} .
$$


Here $J_{k}$ is the Bessel function and

$$
S(m, n ; c)=\sum_{a d=1 \bmod c} e\left(\frac{m a+n c}{c}\right)
$$

is the Kloosterman sum. Since the Petersson weighting leads to a somewhat simpler expression, we will consider that weighting in our example. When passing from the Petersson formula to the expected value, using the Weil bound for the Kloosterman sum and the fact that $J_{k}$ has a $k$ th order zero at 0 , we see that for fixed $m, n$ the sum on the right side of (3.1.47) vanishes as $k \rightarrow \infty$ or $q \rightarrow \infty$.

Let

$$
\delta\left(m_{1}, \ldots, m_{k}\right)=\left\langle\lambda_{f}\left(m_{1}\right) \cdots \lambda_{f}\left(m_{k}\right)\right\rangle .
$$

So in the Petersson weighting, $\delta\left(m_{1}, \ldots, m_{k}\right)$ is the the coefficient $b_{1}$ of $\lambda_{f}(1)=1$ in (3.1.41). One can use the Hecke relations to show by induction that $\delta$ is multiplicative in the sense of 3.1.6. Thus, we only need to know $\delta$ on prime powers.

Lemma 3.1.2. With respect to the Petersson weighting, if $p \nmid q$ then

$$
\begin{aligned}
\delta\left(p^{m_{1}}, \ldots, p^{m_{k}}\right) & =\frac{2}{\pi} \int_{0}^{\pi} \sin ^{2} \theta \prod_{j=1}^{k} \frac{\sin \left(m_{j}+1\right) \theta}{\sin \theta} d \theta \\
& =\frac{2}{\pi} \int_{0}^{\pi} \sin ^{2} \theta \prod_{j=1}^{k} \frac{e^{i\left(m_{j}+1\right) \theta}-e^{-i\left(m_{j}+1\right) \theta}}{e^{i \theta}-e^{-i \theta}} d \theta .
\end{aligned}
$$

For the unweighted sum we have

$$
\delta\left(p^{m_{1}}, \ldots, p^{m_{k}}\right)=\frac{4}{\pi} \int_{0}^{\pi} \frac{\sin ^{2} \theta}{1-\frac{2 \cos \theta}{\sqrt{p}}+\frac{1}{p}} \prod_{j=1}^{k} \frac{\sin \left(m_{j}+1\right) \theta}{\sin \theta} d \theta .
$$

If $p \mid q$ then $\delta\left(p^{m_{1}}, \ldots, p^{m_{k}}\right)=0$ unless $m_{1}=\cdots=m_{k}=0$.

Proof. We only give the details for (3.1.50). Beginning from

$$
\mathcal{L}_{p}(x)=\sum_{j=0}^{\infty} \lambda_{f}\left(p^{j}\right) x^{j}=\left(1-e^{i \theta_{p, f}} x\right)^{-1}\left(1-e^{-i \theta_{p, f}} x\right)^{-1}
$$

we have $\lambda_{f}\left(p^{j}\right)=\frac{\sin (j+1) \theta_{f, p}}{\sin \theta_{f, p}}=U_{j}\left(\cos \theta_{f, p}\right)$ where $U_{j}$ is the usual Tchebychev polynomial. Then $\delta\left(p^{m_{1}}, \ldots, p^{m_{k}}\right)=c_{0}$ where

$$
U_{m_{1}} U_{m_{2}} \ldots U_{m_{k}}=\sum_{e \geq 0} c_{e} U_{e}
$$

If we evaluate both sides of this equation at $\cos \theta$ and integrate from 0 to $\pi$ with respect to the measure $\frac{2}{\pi} \sin ^{2} \theta d \theta$, then the result follows from the orthogonality of the Tchebychev polynomials with respect to this measure.

\section{The family of coefficients of Maass newforms.}

$$
\mathcal{F}_{M}(q)=\left\{f(n)=\lambda_{f}(n): \sqrt{y} \sum \lambda_{f}(n) K_{i R}(2 \pi|n| y) e^{2 \pi i n x} \in H(q)\right\}
$$


where $H(q)$ the set of Maass newforms on $\Gamma_{0}(q)$. A good reference for these functions is Iwaniec [Iw2. The orthogonality relation is derived from the Kuznetsov trace formula. See Chapter 9 of [w2].

3.2. Families of $L$-functions. We use a family of characters to create a family of $L$ functions in the following manner.

Begin with a fixed primitive $L$-function

$$
\mathcal{L}(s)=\sum_{n=1}^{\infty} \frac{a_{n}}{n^{s}}=\prod_{p} \mathcal{L}_{p}\left(\frac{1}{p^{s}}\right), \quad(\sigma>1) .
$$

We assume that

$$
\mathcal{L}_{p}(x)=\sum_{n=0}^{\infty} a_{p^{n}} x^{n}=\prod_{j=1}^{w}\left(1-\gamma_{p, j} x\right)^{-1},
$$

where $w$ is the degree of $\mathcal{L}$ and where $\left|\gamma_{p, j}\right|=0$ or 1 . Assume $\mathcal{L}(s)$ satisfies functional equation

$$
\mathcal{L}(s)=\varepsilon \mathcal{X}(s) \overline{\mathcal{L}}(1-s),
$$

as described in Section 1.1.

We create a family of $L$-functions by twisting $\mathcal{L}$ by a family of characters. Let $\mathcal{F}=\{f\}$ be a family of characters, with the properties described in Section 3.1. The twist of $\mathcal{L}$ by $f$ is denoted by $\mathcal{L}(s, f)$ and is given by a Rankin-Selberg convolution:

$$
\mathcal{L}(s, f)=\prod_{p} \prod_{i=1}^{v} \prod_{j=1}^{w}\left(1-\beta_{p, i} \gamma_{p, j} / p^{s}\right)^{-1}=\sum_{n=1}^{\infty} \frac{a_{n}(f)}{n^{s}} .
$$

Note that if $w=1$ or $v=1$, as will be the case in our detailed examples,

$$
\mathcal{L}(s, f)=\sum_{n=1}^{\infty} \frac{a_{n} f(n)}{n^{s}} .
$$

We require that $\mathcal{L}(s, f)$ is an $L$-function. That is, our family of $L$-functions must consist of $L$-functions! In particular, $\mathcal{L}(s, f)$ satisfies a functional equation

$$
\mathcal{L}(s, f)=\varepsilon_{f} \mathcal{X}_{f}(s) \overline{\mathcal{L}}(1-s, f),
$$

as described in Section 1.1. As part of our definition of "family", we make a restrictive, but natural, assumption on $\mathcal{X}_{f}$. We have $\mathcal{X}_{f}(s)=\overline{\gamma_{f}}(1-s) / \gamma_{f}(s)$ where

$$
\gamma_{f}(s)=Q_{f}^{s} \prod_{j=1}^{w} \Gamma\left(\frac{1}{2} s+\mu_{j, f}\right) .
$$

We assume that $w$ is constant, and each of $Q_{j}, \Re\left(\mu_{j, f}\right)$, and $\Im\left(\mu_{j, f}\right)$ is a monotonic function of the conductor $c(f)$. In practice this will mean that each of those quantities will either be constant or will be tending to infinity with the conductor.

For example, the collection of all real primitive Dirichlet $L$-functions $L\left(s, \chi_{d}\right)$ is not a family, because for $d>0$ we have $\mu_{1, d}=0$ and for $d<0$ we have $\mu_{1, d}=1$, so $\mu_{1, d}$ is not a monotonic function of $c\left(\chi_{d}\right)$. So we consider these two families separately. 
Finally, we will make use of an approximate functional equation of shape

$$
\mathcal{L}(s, f)=\sum \frac{a_{n}(f)}{n^{s}}+\varepsilon_{f} \mathcal{X}_{f}(s) \sum \frac{\overline{a_{n}(f)}}{n^{1-s}}+\text { remainder } .
$$

Note: we are not claiming that the "remainder" in the above equation is small, nevertheless we will ignore the remainder in our calculations.

\section{A RECIPE FOR CONJECTURING MOMENTS, With EXAMPLES}

We give a general recipe for conjecturing the moments of a primitive family of $L$ functions, and then apply the recipe to several interesting examples.

4.1. The general recipe. Suppose $\mathcal{L}$ is an $L$-function and $f$ is a character with conductor $c(f)$, as described in Section 3. So

$$
Z_{\mathcal{L}}(s, f)=\varepsilon_{f}^{-\frac{1}{2}} \mathcal{X}_{f}(s)^{-\frac{1}{2}} \mathcal{L}(s, f),
$$

which satisfies the functional equation

$$
Z_{\mathcal{L}}(s, f)=\overline{Z_{\mathcal{L}}}(1-s, f),
$$

so $Z_{\mathcal{L}}(s, f)$ is real on the $\frac{1}{2}$-line. Note that the square root of $\varepsilon_{f}$ involves a choice which must be made consistently.

We consider the moment

$$
\sum_{f \in \mathcal{F}} Z_{\mathcal{L}}\left(\frac{1}{2}+\alpha_{1}, f\right) \ldots Z_{\mathcal{L}}\left(\frac{1}{2}+\alpha_{k}, f\right) g(c(f))
$$

where $g$ is a suitable test function. The recipe below also applies to averages of products of $\mathcal{L}\left(\frac{1}{2}+\alpha, f\right)$. The sum is an integral when $\mathcal{F}=\mathcal{F}_{t}$.

Here is a recipe for conjecturing a formula for the above moment:

(1) Start with a product of $k$ shifted $L$-functions:

$$
Z_{f}\left(s, \alpha_{1}, \ldots, \alpha_{k}\right)=Z_{\mathcal{L}}\left(s+\alpha_{1}, f\right) \ldots Z_{\mathcal{L}}\left(s+\alpha_{k}, f\right) .
$$

As we will demonstrate in our examples, the recipe applies to the $Z$-function as well as the $L$-function.

(2) Replace each $L$-function with the two terms from its approximate functional equation (3.2.8), ignoring the remainder term. Multiply out the resulting expression to obtain $2^{k}$ terms. Write those terms as

$$
\text { (product of } \varepsilon_{f} \text { factors)(product of } \mathcal{X}_{f} \text { factors) } \sum_{n_{1}, \ldots, n_{k}} \text { (summand). }
$$

(3) Replace each product of $\varepsilon_{f}$-factors by its expected value when averaged over the family.

(4) Replace each summand by its expected value when averaged over the family.

(5) Complete the resulting sums, and call the total $M\left(s, \alpha_{1}, \ldots, \alpha_{2 k}\right)$.

(6) The conjecture is

$$
\sum_{f \in \mathcal{F}} Z_{f}\left(\frac{1}{2}, \alpha_{1}, \ldots, \alpha_{2 k}\right) g(c(f))=\sum_{f \in \mathcal{F}} M_{f}\left(\frac{1}{2}, \alpha_{1}, \ldots, \alpha_{2 k}\right)\left(1+O\left(e^{\left(-\frac{1}{2}+\varepsilon\right) c(f)}\right)\right) g(c(f)),
$$

for all $\varepsilon>0$, where $g$ is a suitable weight function. 
In other words, $Z_{f}(s, \alpha)$ and $M_{f}(s, \alpha)$ have the same value distribution if averaged over a sufficiently large portion of the family. Note that the dependence of $M_{f}$ on $f$ only occurs in the product of $\mathcal{X}_{f}$ factors.

As we mentioned earlier, some of the individual steps in this recipe cannot be rigorously justified. Only by using the entire recipe does one arrive at a reasonable conjecture. In particular, we ignore off-diagonal terms which actually make a contribution. However, comparison with examples in the literature, random matrix moments, and numerical data, suggests that the various errors in our recipe all cancel. The underlying cause for this remains a mystery.

We will apply the recipe to several examples, but first we do the initial steps of the recipe in some generality.

For each $Z_{\mathcal{L}}$ substitute the expression in (4.1.1). After replacing each $\mathcal{L}(s, f)$ by its approximate functional equation (3.2.8), multiply out the product. A typical term is a product of $k$ sums arising from either the first piece or the second piece of the approximate functional equation. Consider a term where we have $\ell$ factors from the first piece of an approximate functional equation and $k-\ell$ factors from the second piece. To take one specific example, suppose it is the first $\ell$ factors from which we choose the first piece of the approximate functional equation, and the last $k-\ell$ factors from which we take the second piece of the approximate functional equation:

$$
\begin{aligned}
\varepsilon_{f}^{-\frac{\ell}{2}} \mathcal{X}_{f}(s & \left.+\alpha_{1}\right)^{-\frac{1}{2}} \cdots \mathcal{X}_{f}\left(s+\alpha_{\ell}\right)^{-\frac{1}{2}} \sum_{n_{1}} \frac{a_{n_{1}}(f)}{n_{1}^{s+\alpha_{1}}} \cdots \sum_{n_{\ell}} \frac{a_{n_{\ell}}(f)}{n_{\ell}^{s+\alpha_{\ell}}} \\
& \times \varepsilon_{f}^{\frac{1}{2}(k-\ell)} \mathcal{X}_{f}\left(s+\alpha_{\ell+1}\right)^{\frac{1}{2}} \cdots \mathcal{X}_{f}\left(s+\alpha_{k}\right)^{\frac{1}{2}} \sum_{n_{\ell+1}} \frac{\overline{a_{n_{\ell+1}}(f)}}{n_{\ell+1}^{1-s-\alpha_{\ell+1}}} \cdots \sum_{n_{k}} \frac{\overline{a_{n_{k}}(f)}}{n_{k}^{1-s-\alpha_{k}}} .
\end{aligned}
$$

Rearranging this expression and using the fact that $\mathcal{X}_{f}(s)=\mathcal{X}_{f}(1-s)^{-1}$, we have

$$
\begin{aligned}
\varepsilon_{f}^{\frac{k}{2}-\ell} \prod_{j=1}^{\ell} \mathcal{X}_{f}\left(s+\alpha_{j}\right)^{-\frac{1}{2}} \prod_{j=\ell+1}^{k} \mathcal{X}_{f}\left(1-s-\alpha_{j}\right)^{-\frac{1}{2}} & \\
& \times \sum_{n_{1}, \ldots, n_{k}} \frac{a_{n_{1}}(f) \cdots a_{n_{\ell}}(f) \overline{a_{n_{\ell+1}}(f) \cdots a_{n_{k}}(f)}}{n_{1}^{s+\alpha_{1}} \cdots n_{\ell}^{s+\alpha_{\ell}} n_{\ell+1}^{1-s-\alpha_{\ell+1}} \cdots n_{k}^{1-s-\alpha_{k}}}
\end{aligned}
$$

A little trick: since we will eventually set $s=\frac{1}{2}$, we replace the above expression by

$$
\begin{aligned}
\varepsilon_{f}^{\frac{k}{2}-\ell} \prod_{j=1}^{\ell} \mathcal{X}_{f}\left(s+\alpha_{j}\right)^{-\frac{1}{2}} \prod_{j=\ell+1}^{k} \mathcal{X}_{f}\left(s-\alpha_{j}\right)^{-\frac{1}{2}} & \\
& \times \sum_{n_{1}, \ldots, n_{k}} \frac{a_{n_{1}}(f) \cdots a_{n_{\ell}}(f) \overline{a_{n_{\ell+1}}(f) \cdots a_{n_{k}}(f)}}{n_{1}^{s+\alpha_{1}} \cdots n_{\ell}^{s+\alpha_{\ell}} n_{\ell+1}^{s-\alpha_{\ell+1}} \cdots n_{k}^{s-\alpha_{k}}}
\end{aligned}
$$

It is expression (4.1.9), and the corresponding pieces from the other terms when multiplying out the approximate functional equation, which will appear in the final conjecture, evaluated at $s=\frac{1}{2}$. 
Now consider the product of $\varepsilon_{f}$ factors $\varepsilon_{f}^{\frac{k}{2}-\ell}$, which according to the recipe should be replaced by its expected value. An important issue is the choice of the square root. We believe that there is a natural choice of $\varepsilon_{f}^{\frac{1}{2}}$ so that the following hold.

- Unitary case: the $\varepsilon_{f}$ are uniformly distributed on the unit circle, and $\left\langle\varepsilon_{f}^{\frac{k}{2}-\ell}\right\rangle=0$ unless $\frac{k}{2}-\ell=0$. In particular, $k$ must be even. There will be $\left(\begin{array}{c}k \\ k / 2\end{array}\right)$ terms in the final answer.

- Orthoconal case: $\varepsilon_{f}$ is constant $(1$ or -1$)$ over the family, or $\varepsilon_{f}=1$ for approximately half the $f$ and $\varepsilon_{f}=-1$ for the other half. We have $\left\langle\varepsilon_{f}^{\frac{k}{2}-\ell}\right\rangle=0$ unless $\frac{k}{2}-\ell$ is even. In particular, $k$ must be even and there will be $2^{k-1}$ terms in the final answer.

- Symplectic case: $\varepsilon_{f}=1$ for all $f$, and $\left\langle\varepsilon_{f}^{\frac{k}{2}-\ell}\right\rangle=1$ for all $k$ and $\ell$. There is no restriction and there will be $2^{k}$ terms in the final answer.

Note that if we are considering the $L$-function, instead of the $Z$-function, then the issue of $\varepsilon_{f}^{\frac{1}{2}}$ does not arise and the calculation is somewhat easier. See (4.5.4) and the discussion following. Also note that in the Unitary and Orthogonal cases, odd powers of the $Z$-function will average to zero, while odd powers of the $L$-function will not.

The recipe now says to replace the summand by its expected value when averaged over the family. That is, we replace

$$
a_{n_{1}}(f) \ldots a_{n_{\ell}}(f) \overline{a_{n_{\ell+1}}(f) \ldots a_{n_{k}}(f)}
$$

by its expected value when averaged over the family. In practice, this will be of the form

$$
c(\mathcal{F}) \delta_{\ell}\left(n_{1}, \ldots, n_{k}\right)
$$

where $c(\mathcal{F})$ depends only on the family, and where the $\delta_{\ell}$ are multiplicative functions, i.e.

$$
\delta_{\ell}\left(m_{1} n_{1}, \ldots, m_{k} n_{k}\right)=\delta_{\ell}\left(m_{1}, \ldots, m_{k}\right) \delta_{\ell}\left(n_{1}, \ldots, n_{k}\right)
$$

whenever $\left(m_{1} \cdots m_{k}, n_{1} \cdots n_{k}\right)=1$.

The final step is to extend the range of summation. This produces one term in the conjecture. By considering the other terms when multiplying out the approximate functional equations, one arrives at a conjecture for the original mean value.

Although the above steps have produced an answer, it is not written in a particularly usable form. There are three more steps to put the conjecture in the form of Conjecture 1.5.1. writing the main terms as an Euler product, identifying the polar part, and expressing the combinatorial sum as a multiple integral.

Since the $\delta_{\ell}$ are multiplicative, we can write the main term as an Euler product. Specifically,

$$
\sum_{n_{1}, \ldots, n_{k}=1}^{\infty} \frac{\delta_{\ell}\left(n_{1}, \ldots, n_{k}\right)}{n_{1}^{s+\alpha_{1}} \cdots n_{k}^{s+\alpha_{k}}}=\prod_{p} \sum_{e_{1}, \ldots, e_{k}=0}^{\infty} \frac{\delta_{\ell}\left(p^{e_{1}}, \ldots, p^{e_{k}}\right)}{p^{e_{1}\left(s+\alpha_{1}\right)+\cdots+e_{k}\left(s+\alpha_{k}\right)}}
$$

assuming that $\Re s$ is sufficiently large.

Next we determine the leading order poles. It usually turns out that $\delta_{\ell}\left(p^{e_{1}}, \ldots, p^{e_{k}}\right)=0$ when $\sum e_{j}=1$. Thus, the first poles come from those terms where $\sum e_{j}=2$. This happens 
in two ways, so the rightmost poles are the same as the poles of

$$
\prod_{1 \leq i<j \leq k} \prod_{p}\left(1+\frac{\delta_{\ell, i, j}(p, p)}{p^{2 s+\alpha_{i}+\alpha_{j}}}\right) \times \prod_{j=1}^{k} \prod_{p}\left(1+\frac{\delta_{\ell, j}\left(p^{2}\right)}{p^{2 s+2 \alpha_{j}}}\right) .
$$

In practice the first factor has simple poles at $\frac{1}{2}-\frac{1}{2}\left(\alpha_{i}+\alpha_{j}\right)$, and the second factor is either regular in a neighborhood of $\sigma=\frac{1}{2}$, or else it has a simple pole at $s=\frac{1}{2}-\alpha_{j}$. Accordingly, we factor out either

$$
\prod_{1 \leq i<j \leq k} \zeta\left(2 s+\alpha_{i}+\alpha_{j}\right) \quad \text { or } \quad \prod_{1 \leq i \leq j \leq k} \zeta\left(2 s+\alpha_{i}+\alpha_{j}\right) .
$$

The remainder is the $A_{k}$ in our conjectures, and it is regular in a neighborhood of $\sigma=\frac{1}{2}$.

Having identified the polar part of our main terms, we can apply the lemmas in Section 2.5 to express the sum of terms as a contour integral. The result is an expression similar to Conjecture 1.5 .1

We have already seen this procedure in Section 2.2 for the case of mean values of the zeta-function. In the following sections we carry out example calculations for families of each of the three symmetry types.

4.2. Unitary: moments of a primitive $L$-function. The recipe for mean values in Section 2.1 is a special case of the general recipe. To see this, note that if $f_{t} \in \mathcal{F}_{t}$ then $f_{t}(n)=n^{-i t}$, so $\mathcal{L}\left(s, f_{t}\right)=\mathcal{L}(s+i t)$. From the functional equation $\mathcal{L}(s)=\varepsilon \mathcal{X}(s) \overline{\mathcal{L}}(1-s)$ we obtain the functional equation

$$
\mathcal{L}\left(s, f_{t}\right)=\varepsilon_{t} \mathcal{X}_{t}(s) \overline{\mathcal{L}}\left(1-s, f_{t}\right)
$$

where

$$
\varepsilon_{t}=\varepsilon \mathcal{X}\left(\frac{1}{2}+i t\right), \quad \text { and } \quad \mathcal{X}_{t}(s)=\frac{\mathcal{X}(s+i t)}{\mathcal{X}\left(\frac{1}{2}+i t\right)} .
$$

Note that these satisfy the requirements $\left|\mathcal{X}_{t}\left(\frac{1}{2}+i y\right)\right|=1$ for $y$ real, with $\mathcal{X}_{t}\left(\frac{1}{2}\right)=1$ and $\left|\varepsilon_{t}\right|=$ 1. Also note that the $\log$ conductor of $\mathcal{L}\left(s, f_{t}\right)$, defined as $\left|\left(\varepsilon_{t} \mathcal{X}_{t}\right)^{\prime}\left(\frac{1}{2}\right)\right|$, equals $\left|\mathcal{X}^{\prime}\left(\frac{1}{2}+i t\right)\right|$, in agreement with the usual notion of conductor in $t$-aspect.

Replacing the product of $\varepsilon_{t}$-factors by their expected value is the same "keep the terms where the product of the $\chi$-factors is not oscillating." Thus, after multiplying out the approximate functional equations there will be $\left(\begin{array}{c}2 k \\ k\end{array}\right)$ terms which contribute. In each of those terms replacing the summand by its expected value is the same as "keeping the diagonal." Thus, we arrive at the same conjecture as before.

4.3. Unitary: all Dirichlet $L$-functions. We apply our recipe to conjecture the average

$$
\sum_{\substack{\chi \bmod q \\ \chi \text { even or odd }}}^{*} Z_{\chi}\left(\frac{1}{2} ; \alpha_{1}, \ldots, \alpha_{2 k}\right),
$$

where the sum is over either the even or the odd primitive Dirichlet characters mod $q$ and

$$
Z_{\chi}\left(s ; \alpha_{1}, \ldots, \alpha_{2 k}\right)=Z\left(s+\alpha_{1}, \chi\right) \ldots Z\left(s+\alpha_{2 k}, \chi\right) .
$$

Here $Z\left(s+\alpha_{k}, \chi\right)=\left(\varepsilon_{\chi} X_{\chi}(s)\right)^{-\frac{1}{2}} L(s, \chi)$ where $L(s, \chi)=\varepsilon_{\chi} X_{\chi}(s) L(1-s, \chi)$. Note that $\varepsilon_{\chi}=\tau(\chi) q^{-\frac{1}{2}}$, which is uniformly distributed on the unit circle. 
Following the general discussion in Section 4.1, equation (4.1.9) specializes in this case to (4.3.3) $\varepsilon_{\chi}^{k-\ell} \prod_{j=1}^{\ell} X_{\chi}\left(\frac{1}{2}+\alpha_{j}\right)^{-\frac{1}{2}} \prod_{j=\ell+1}^{2 k} X_{\chi}\left(\frac{1}{2}-\alpha_{j}\right)^{-\frac{1}{2}} \sum_{n_{1}, \ldots, n_{2 k}} \frac{1}{n_{1}^{\frac{1}{2}+\alpha_{1}} \cdots n_{2 k}^{\frac{1}{2}-\alpha_{2 k}}} \chi\left(n_{1}\right) \cdots \bar{\chi}\left(n_{2 k}\right)$.

According to the recipe, we replace $\varepsilon_{\chi}^{k-\ell}$ by its expected value. Since the $\varepsilon_{\chi}$ are uniformly distributed on the unit circle, the expected value is 1 if $\ell=k$ and 0 otherwise, so we keep $\left(\begin{array}{c}2 k \\ k\end{array}\right)$ terms.

Next we replace the summand by its expected value, which is

$$
\begin{aligned}
\delta\left(n_{1}, \ldots, n_{2 k}\right) & =\left\langle\chi\left(n_{1}\right) \cdots \chi\left(n_{k}\right) \bar{\chi}\left(n_{k+1}\right) \cdots \bar{\chi}\left(n_{2 k}\right)\right\rangle \\
& = \begin{cases}1 & n_{1} \cdots n_{k}=n_{k+1} \cdots n_{2 k} \\
0 & \text { otherwise. }\end{cases}
\end{aligned}
$$

The above is almost identical to the conjectures obtained for the mean values, in $t$-aspect, for a primitive $L$-function. So one obtains the same formulas as appear in Conjectures [1.5.1] and 2.5.4, the only changes being that one omits the factors $p \mid q$ in the Euler product $A_{k}$, and one must use the factors $X_{\chi}\left(\frac{1}{2} \pm \alpha_{j}\right)^{-\frac{1}{2}}$. Specifically, in Conjectures 1.5.1 and 2.5.4 a simplification occurred by use of equations (2.2.4) and (2.3.6). If those conjectures are written in terms of $\prod X\left(\frac{1}{2} \pm z_{j}\right)^{-\frac{1}{2}}$, then the Dirichlet $L$-function moment conjecture would be obtained by substituting with $\prod X_{\chi}\left(\frac{1}{2} \pm z_{j}\right)^{-\frac{1}{2}}$ Note that we are considering the averages over the even and odd primitive characters separately, so in the sum $X_{\chi}$ only depends on the conductor of $\chi$. See the comments following the Theorems in Section 1.5 for more discussion on these $X$-factors and conductors.

4.4. Symplectic and Orthogonal: quadratic twists of a real $L$-function. Next we consider what happens when we average the shifts of central values of $\mathcal{L}(s)$ twisted by the family of quadratic characters $\chi_{d}(n)=\left(\frac{d}{n}\right), d<0$ a fundamental discriminant. Here $\chi_{d}(n)=\left(\frac{d}{n}\right)$ is the Kronecker symbol which is a primitive Dirichlet character of conductor $|d|$. We will see that the family can be either Symplectic or Orthogonal, depending on the particular $L$-function we start with.

Again, let

$$
\mathcal{L}(s)=\sum_{n=1}^{\infty} \frac{a_{n}}{n^{s}}=\prod_{p} \mathcal{L}_{p}\left(1 / p^{s}\right)
$$

be a primitive $L$-function and note that

$$
\mathcal{L}\left(s, \chi_{d}\right)=\sum_{n=1}^{\infty} \frac{a_{n}\left(\frac{d}{n}\right)}{n^{s}} .
$$

We assume that $\mathcal{L}$ is real, i.e. $\mathcal{L}=\overline{\mathcal{L}}$, as this case is relatively easy to deal with from a fairly general perspective. Thus,

$$
\mathcal{L}(s)=\varepsilon \mathcal{X}(s) \mathcal{L}(1-s),
$$


where $\varepsilon= \pm 1$. The twisted $L$-function is expected to satisfy a functional equation of the form

$$
\mathcal{L}\left(s, \chi_{d}\right)=\varepsilon_{d} \mathcal{X}_{d}(s) \mathcal{L}\left(1-s, \chi_{d}\right)
$$

It is further expected that

$$
\mathcal{X}_{d}(s)=|d|^{w\left(\frac{1}{2}-s\right)} X(s, d)
$$

where there are only finitely many possibilities for $X(s, d)$. By our definition of "family" we require that the parameters in the functional equation be monotonic functions of the conductor. Since there are only finitely many choices for $X(s, d)$, we must restrict to averages over sets of $d$ for which $X(s, d)$ is constant (as a function of $d$ ). In the situation described here, it is believed that there exists an integer $N$, depending on $\mathcal{L}$, such that $\varepsilon_{d}$ and $X(s, d)$ only depend on the sign of $d$ and on $(d \bmod N)$. Thus, we will consider the averages

$$
\sum_{\substack{d<0 \\ d \equiv a \bmod N}}^{*} L_{d}\left(\frac{1}{2} ; \alpha_{1}, \ldots, \alpha_{k}\right) g(|d|),
$$

(the following analysis holds also for $d>0$ ) where $\sum^{*}$ denotes a sum over fundamental discriminants $d$, and

$$
L_{d}\left(s ; \alpha_{1}, \ldots, \alpha_{k}\right)=Z_{\mathcal{L}}\left(s+\alpha_{1}, \chi_{d}\right) \ldots Z_{\mathcal{L}}\left(s+\alpha_{k}, \chi_{d}\right) .
$$

Note that $\varepsilon_{d}=\varepsilon_{a}$, which may depend on the $\operatorname{sign}$ of $d$. If $N$ is even we are insisting further that it be divisible by at least 8 .

Following the general discussion in Section 4.1, equation (4.1.9) specializes in this case to

$$
\varepsilon_{f}^{\frac{k}{2}-\ell} \prod_{j=1}^{\ell} \mathcal{X}_{d}\left(s+\alpha_{j}\right)^{-\frac{1}{2}} \prod_{j=\ell+1}^{k} \mathcal{X}_{d}\left(s-\alpha_{j}\right)^{-\frac{1}{2}} \sum_{n_{1}, \ldots, n_{k}} \frac{a_{n_{1}} \cdots a_{n_{k}}}{n_{1}^{s+\alpha_{1}} \cdots n_{k}^{s-\alpha_{k}}} \chi_{d}\left(n_{1}\right) \cdots \chi_{d}\left(n_{k}\right)
$$

According to the recipe, we replace $\varepsilon_{f}^{\frac{k}{2}-\ell}$ by its expected value. We have assumed (by our choice of $a \bmod N$ ) that $\varepsilon_{d}=\varepsilon_{a}$ for all $d$, so the expected value is $\varepsilon_{a}^{\frac{k}{2}-\ell}$ and we will have a contribution from all $2^{k}$ terms. (That expression is more transparent is you separately consider the cases $\varepsilon_{a}=1$ and $\varepsilon_{a}=-1$ ).

The next step in the recipe is to replace the summand by its expected value. Since $\chi_{d}\left(n_{1}\right) \cdots \chi_{d}\left(n_{k}\right)=\chi_{d}\left(n_{1} \cdots n_{k}\right)$, from equation (3.1.21) we have the expected value

$$
\left\langle\chi_{d}\left(n_{1}\right) \cdots \chi_{d}\left(n_{k}\right)\right\rangle= \begin{cases}\chi_{a}(g) \prod_{p \mid \square}\left(1+\frac{1}{p}\right)^{-1} & n_{1} \cdots n_{k}=g \square \\ 0 & \text { otherwise }\end{cases}
$$

where $(N, \square)=1$, and all the prime factors of $g$ also being prime factors of $N$. So the contribution from the term where we use the first part of the approximate functional equation for the first $\ell$ factors, and the second part for the rest, is

$$
\varepsilon_{d}^{\frac{k}{2}-\ell} \prod_{j=1}^{\ell} \mathcal{X}_{d}\left(s+\alpha_{j}\right)^{-\frac{1}{2}} \prod_{j=\ell+1}^{k} \mathcal{X}_{d}\left(s-\alpha_{j}\right)^{-\frac{1}{2}} R_{k, N}\left(s ; \alpha_{1}, \ldots, \alpha_{\ell},-\alpha_{\ell+1}, \ldots,-\alpha_{k}\right)
$$

where

$$
R_{k, N}\left(s ; \alpha_{1}, \ldots, \alpha_{k}\right)=\sum_{g \square} \chi_{a}(g) \sum_{n_{1} \cdots n_{k}=g \square} \frac{a_{n_{1}} \cdots a_{n_{k}}}{n_{1}^{s+\alpha_{1}} \cdots n_{k}^{s+\alpha_{k}}} \prod_{p \mid \square}\left(1+\frac{1}{p}\right)^{-1}
$$


Adding up all $2^{k}$ terms we obtain

$$
M\left(s ; \alpha_{1}, \ldots, \alpha_{k}\right)=\sum_{\epsilon_{i}= \pm 1} \operatorname{sign}\left(\left\{\epsilon_{j}\right\}\right) \prod_{j=1}^{k} \mathcal{X}_{d}\left(\frac{1}{2}+\epsilon_{j} \alpha_{j}\right)^{-\frac{1}{2}} R_{k, N}\left(s ; \epsilon_{1} \alpha_{1}, \ldots, \epsilon_{k} \alpha_{k}\right),
$$

where

$$
\operatorname{sign}\left(\left\{\epsilon_{j}\right\}\right)= \begin{cases}1 & \varepsilon_{a}=1 \\ (-1)^{\frac{1}{2} \sum \epsilon_{i}} & \varepsilon_{a}=-1\end{cases}
$$

So the recipe has produced the conjecture

$$
\sum_{\substack{d<0 \\ d \equiv a \bmod N}}^{*} L_{d}\left(\frac{1}{2}, \alpha_{1}, \ldots, \alpha_{k}\right) g(d)=\sum_{\substack{d<0 \\ d \equiv a \bmod N}}^{*} M\left(\frac{1}{2} ; \alpha_{1}, \ldots, \alpha_{k}\right)\left(1+O\left(|d|^{-\frac{1}{2}+\varepsilon}\right)\right) g(d) .
$$

To put the conjecture in a more useful form, we now write $R_{k, N}$ as an Euler product, and then express the main term as a contour integral.

We have $R_{k, N}=\prod_{p} R_{k, N, p}$, which naturally separates into a product over the primes which divide $N$ and a product over the primes which do not divide $N$. The $p$-factor when $p \nmid N$ is

$$
\begin{aligned}
R_{k, N, p}(s) & =\left(1+\left(1+\frac{1}{p}\right)^{-1} \sum_{j=1}^{\infty} \sum_{e_{1}+\ldots e_{k}=2 j} \prod_{i=1}^{k} \frac{a_{p^{e_{i}}}}{p^{e_{i}\left(s+\alpha_{i}\right)}}\right) \\
& =\left(1+\frac{1}{p}\right)^{-1}\left(\frac{1}{p}+\sum_{j=0}^{\infty} \sum_{e_{1}+\ldots e_{k}=2 j} \prod_{i=1}^{k} \frac{a_{p^{e_{i}}}}{p^{e_{i}\left(s+\alpha_{i}\right)}}\right) \\
& =\left(1+\frac{1}{p}\right)^{-1}\left(\frac{1}{p}+\frac{1}{2}\left(\prod_{j=1}^{k} \mathcal{L}_{p}\left(\frac{1}{p^{s+\alpha_{j}}}\right)+\prod_{j=1}^{k} \mathcal{L}_{p}\left(\frac{-1}{p^{s+\alpha_{j}}}\right)\right)\right) .
\end{aligned}
$$

Similarly, the $p$-factor when $p \mid N$ is

$$
R_{k, N, p}=\prod_{j=1}^{k} \mathcal{L}_{p}\left(\frac{\chi_{a}(p)}{p^{s+\alpha_{j}}}\right) .
$$

The above expression will enable us to locate the leading poles of $R_{k, N}$. Consider the expansion of $R_{k, N, p}($ for $p \nmid N)$ in powers of $1 / p$. The expansion is of the form

$$
\begin{aligned}
& 1+\sum_{j=1}^{k} \frac{a_{p^{2}}}{p^{2 s+2 \alpha_{j}}}+\sum_{1 \leq i<j \leq k} \frac{\left(a_{p}\right)^{2}}{p^{2 s+\alpha_{i}+\alpha_{j}}}+O\left(p^{-1-2 s+\varepsilon}\right)+O\left(p^{-3 s+\varepsilon}\right) \\
& (4.4 .17)=\prod_{j=1}^{k}\left(1+\frac{a_{p^{2}}}{p^{2 s+\alpha_{j}}}\right) \times \prod_{1 \leq i<j \leq k}\left(1+\frac{\left(a_{p}\right)^{2}}{p^{2 s+\alpha_{i}+\alpha_{j}}}\right) \times\left(1+O\left(p^{-1-2 s+\varepsilon}\right)+O\left(p^{-3 s+\varepsilon}\right)\right)
\end{aligned}
$$

We assume that

$$
\prod_{p}\left(1+\frac{\left(a_{p}\right)^{2}}{p^{s}}\right)
$$


has a simple pole at $s=1$. This is conjectured to be equivalent to $\mathcal{L}(s)$ being a primitive $L$ function, and this is the key place where the assumption of primitivity enters the calculation. We also assume that

$$
\prod_{p}\left(1+\frac{a_{p^{2}}}{p^{s}}\right)
$$

has a pole of order $\delta=0$ or 1 at $s=1$.

In general, $\delta$ is expected to be 0 or 1 according to whether the symmetric square $L$ function of $\mathcal{L}(s)$ is analytic at $s=1$ or has a simple pole at $s=1$. If $\mathcal{L}(s)$ is a degree 1 $L$-function, (that is, the Riemann $\zeta$-function or a Dirichlet $L$-function), then $\delta=1$. If $\mathcal{L}(s)$ is associated to a $G L(2)$ automorphic form, then $\delta=0$ in general (except possibly when $\mathcal{L}$ is a dihedral Artin $L$-function associated to an weight 1 modular form).

Note that $\prod_{p}\left(1+O\left(p^{-1-2 s}\right)+O\left(p^{-3 s}\right)\right)$ is regular for $\sigma>\frac{1}{3}$. Thus the total order of the pole of the above product at $s=\frac{1}{2}$ when $\alpha_{1}=\cdots=\alpha_{k}=0$ is $\frac{1}{2} k(k-1)+\delta k$. Accordingly, we factor out appropriate zeta-factors and write the above product as

$$
R_{k, N}(s)=\prod_{1 \leq i<j \leq k} \zeta\left(2 s+\alpha_{i}+\alpha_{j}\right) \prod_{p} R_{k, N, p}(s) \prod_{1 \leq i<j \leq k}\left(1-\frac{1}{p^{2 s+\alpha_{i}+\alpha_{j}}}\right)
$$

if $\delta=0$, and as

$$
R_{k, N}(s)=\prod_{1 \leq i \leq j \leq k} \zeta\left(2 s+\alpha_{i}+\alpha_{j}\right) \prod_{p} R_{k, N, p}(s) \prod_{1 \leq i \leq j \leq k}\left(1-\frac{1}{p^{2 s+\alpha_{i}+\alpha_{j}}}\right)
$$

if $\delta=1$. In the first case above the family is Orthogonal, and in the second case it is Symplectic.

In summary, we are led to conjecture

$$
\begin{aligned}
& \sum_{\substack{d<0 \\
d \equiv a \bmod N}}^{*} Z_{\mathcal{L}}\left(\frac{1}{2}+\alpha_{1}, \chi_{d}\right) \ldots Z_{\mathcal{L}}\left(\frac{1}{2}+\alpha_{k}, \chi_{d}\right) g(|d|) \\
&=\sum_{\epsilon_{i}= \pm 1} \operatorname{sign}\left(\left\{\epsilon_{i}\right\}\right) \prod_{j=1}^{k} X\left(\frac{1}{2}+\epsilon_{j} \alpha_{j}, a\right)^{-\frac{1}{2}} \sum_{\substack{d<0 \\
d \equiv a \bmod N}}^{*} R_{k, N}\left(\frac{1}{2}, \epsilon_{1} \alpha_{1}, \ldots, \epsilon_{k} \alpha_{k}\right)|d|^{\frac{w}{2} \sum_{j=1}^{k} \epsilon_{j} \alpha_{j}} \\
& \times\left(1+O\left(|d|^{-\frac{1}{2}+\varepsilon}\right)\right) g(|d|) .
\end{aligned}
$$

The analogous sum over $d>0$ leads to a similar conjecture. Here $\operatorname{sign}\left(\left\{\epsilon_{i}\right\}\right)$ is given in (4.4.13) and in either case we can use Lemma 2.5.2 to write the sum as a contour integral.

In the case that $\mathcal{L}(s)$ is the Riemann zeta-function, the above reduces to Conjecture 1.5 .3

4.5. Orthogonal: $L$-functions associated with cusp forms. Recall that the set of primitive newforms $f \in S_{n}\left(\Gamma_{0}(q)\right)$ is denoted by $H_{n}(q)$. In this section we consider the shifted moments of $L_{f}(s)=\sum_{n=1}^{\infty} \lambda_{f}(n) n^{-s}$ near the critical point $s=\frac{1}{2}$ averaged over $f \in H_{n}(q)$. Note that in the language of Section 3.2 these $L$-functions are the twists of $\zeta(s)$ by the family 
of characters $H_{k}(q)$ and would be denoted as $\zeta(s, f)$. However, we use the more common notation here.

The functional equation is

$$
L_{f}(s)=\varepsilon_{f} X(s) L_{f}(1-s)
$$

where $\varepsilon_{f}=-\sqrt{q} \lambda_{f}(q)= \pm 1$.

We consider the "harmonic average"

$$
\sum_{f \in H_{n}(q)}^{\mathrm{h}} L_{f}\left(\frac{1}{2}+\alpha_{1}\right) \ldots L_{f}\left(\frac{1}{2}+\alpha_{k}\right)
$$

which attaches a weight $\langle f, f\rangle^{-1}$ to each summand. That is,

$$
\sum_{f \in H_{n}(q)}^{\mathrm{h}} *=\sum_{f \in H_{n}(q)} * /\langle f, f\rangle .
$$

Following the general discussion in Section 4.1, equation (4.1.9) specializes in this case to

$$
\varepsilon_{f}^{k-\ell} \prod_{j=\ell+1}^{k} X\left(s-\alpha_{j}\right)^{-1} \sum_{n_{1}, \ldots, n_{k}} \frac{\lambda_{f}\left(n_{1}\right) \cdots \lambda_{f}\left(n_{k}\right)}{n_{1}^{s+\alpha_{1}} \cdots n_{k}^{s-\alpha_{k}}} .
$$

According to the recipe, we replace $\varepsilon_{f}^{k-\ell}$ by its expected value. Since $\varepsilon_{f}$ is randomly \pm 1 , the expected value is 0 unless $k-\ell$ is even. Thus, we will have $2^{k-1}$ terms in the final answer.

Next we replace $\lambda_{f}\left(n_{1}\right) \cdots \lambda_{f}\left(n_{k}\right)$ by its expected value. This is given in Lemma 3.1 .2 After factoring into an Euler product and summing the relevant geometric series we see that (4.5.4) contributes $\prod_{j=1}^{k} X\left(s-\alpha_{j}\right)^{-\frac{1}{2}} R\left(\alpha_{1}, \ldots, \alpha_{\ell},-\alpha_{\ell+1}, \ldots,-\alpha_{k}\right)$, where

$$
\begin{aligned}
& R\left(s, \alpha_{1}, \ldots, \alpha_{k}\right) \\
& \quad=\prod_{j=1}^{k} X\left(s+\alpha_{j}\right)^{-\frac{1}{2}} \prod_{p} \frac{2}{\pi} \int_{0}^{\pi} \sin ^{2} \theta \prod_{j=1}^{k} \frac{e^{i \theta}\left(1-\frac{e^{i \theta}}{p^{s+\alpha_{j}}}\right)^{-1}-e^{-i \theta}\left(1-\frac{e^{-i \theta}}{p^{s+\alpha_{j}}}\right)^{-1}}{e^{i \theta}-e^{-i \theta}} d \theta
\end{aligned}
$$

Here remember that $s$ will eventually be set to $\frac{1}{2}$ and $X(s)=X(1-s)^{-1}$. Adding up all $2^{k-1}$ terms we obtain

$$
M\left(s ; \alpha_{1}, \ldots, \alpha_{k}\right)=\prod_{j=1}^{k} X\left(s-\alpha_{j}\right)^{-\frac{1}{2}} \sum_{\substack{\epsilon_{i}= \pm 1 \\ \prod_{j=1}^{k} \epsilon_{j}=1}} R\left(s ; \epsilon_{1} \alpha_{1}, \ldots, \epsilon_{k} \alpha_{k}\right),
$$

so the recipe has produced the conjecture

$$
\begin{aligned}
\sum_{f \in H_{n}(q)}^{\mathrm{h}} L_{f}\left(\frac{1}{2}+\alpha_{1}\right) \ldots L_{f}\left(\frac{1}{2}+\alpha_{k}\right) & =\sum_{f \in H_{n}(q)}^{\mathrm{h}} M\left(\frac{1}{2} ; \alpha_{1}, \ldots, \alpha_{k}\right)\left(1+O(n q)^{-\frac{1}{2}+\varepsilon}\right) \\
& =\left(1+O(n q)^{-\frac{1}{2}+\varepsilon}\right) M\left(\frac{1}{2} ; \alpha_{1}, \ldots, \alpha_{k}\right)
\end{aligned}
$$

Summarizing, we have 
Conjecture 4.5.1. With $A_{k}\left(\alpha_{1}, \ldots, \alpha_{k}\right)$ as in Conjecture 1.5.5, we have

$\sum_{f \in H_{n}(q)}^{\mathrm{h}} L_{f}\left(\frac{1}{2}+\alpha_{1}\right) \ldots L_{f}\left(\frac{1}{2}+\alpha_{k}\right)=$

$$
\begin{aligned}
\prod_{j=1}^{k} X\left(\frac{1}{2}-\alpha_{j}\right)^{-\frac{1}{2}} & \sum_{\substack{\epsilon_{j}= \pm 1 \\
\prod_{j=1}^{k} \epsilon_{j}=1}} \prod_{j=1}^{k} X\left(\frac{1}{2}+\epsilon_{j} \alpha_{j}\right)^{-\frac{1}{2}} \\
& \times \prod_{1 \leq i<j \leq k} \zeta\left(1+\epsilon_{i} \alpha_{i}+\epsilon_{j} \alpha_{j}\right) A_{k}\left(\epsilon_{1} \alpha_{1}, \ldots, \epsilon_{k} \alpha_{k}\right)\left(1+O(n q)^{-\frac{1}{2}+\varepsilon}\right) .
\end{aligned}
$$

For the purpose of considering averages of even forms or odd forms separately, we note that

$$
\begin{aligned}
\sum_{f \in H_{n}(q)}^{\mathrm{h}} \varepsilon_{f} L_{f}\left(\frac{1}{2}+\alpha_{1}\right) \ldots L_{f}\left(\frac{1}{2}+\alpha_{k}\right) & \\
= & X\left(\frac{1}{2}+\alpha_{1}\right) \sum_{f \in H_{2}(q)}^{\mathrm{h}} L_{f}\left(\frac{1}{2}-\alpha_{1}\right) L_{f}\left(\frac{1}{2}+\alpha_{2}\right) \ldots L_{f}\left(\frac{1}{2}+\alpha_{k}\right) \\
& =X\left(\frac{1}{2}+\alpha_{1}\right) M\left(\frac{1}{2} ;-\alpha_{1}, \alpha_{2}, \ldots, \alpha_{k}\right)\left(1+O(n q)^{-\frac{1}{2}+\varepsilon}\right) .
\end{aligned}
$$

By looking at the combinations

$$
\sum_{f \in H_{n}(q)}^{\mathrm{h}} L_{f}\left(\frac{1}{2}+\alpha_{1}\right) \ldots L_{f}\left(\frac{1}{2}+\alpha_{k}\right) \pm \sum_{f \in H_{n}(q)}^{\mathrm{h}} \varepsilon_{f} L_{f}\left(\frac{1}{2}+\alpha_{1}\right) \ldots L_{f}\left(\frac{1}{2}+\alpha_{k}\right)
$$

this leads to

Conjecture 4.5.2. With $A_{k}\left(\alpha_{1}, \ldots, \alpha_{k}\right)$ as in Conjecture 1.5.5, we have

$\sum_{\substack{f \in H_{n}(q) \\ f \text { even }}}^{\mathrm{h}} L_{f}\left(\frac{1}{2}+\alpha_{1}\right) \ldots L_{f}\left(\frac{1}{2}+\alpha_{k}\right)=$

$$
\begin{aligned}
\frac{1}{2} \prod_{j=1}^{k} X\left(\frac{1}{2}-\alpha_{j}\right)^{-\frac{1}{2}} & \sum_{\epsilon_{j}= \pm 1} \prod_{j=1}^{k} X\left(\frac{1}{2}+\epsilon_{j} \alpha_{j}\right)^{-\frac{1}{2}} \\
& \times \prod_{1 \leq i<j \leq k} \zeta\left(1+\epsilon_{i} \alpha_{i}+\epsilon_{j} \alpha_{j}\right) A_{k}\left(\epsilon_{1} \alpha_{1}, \ldots, \epsilon_{k} \alpha_{k}\right)\left(1+O(n q)^{-\frac{1}{2}+\varepsilon}\right),
\end{aligned}
$$

and

$$
\begin{aligned}
& \sum_{\substack{f \in H_{n}(q) \\
f \text { odd }}}^{\mathrm{h}} L_{f}\left(\frac{1}{2}+\alpha_{1}\right) \ldots L_{f}\left(\frac{1}{2}+\alpha_{k}\right) \\
& \qquad \begin{array}{c}
\frac{1}{2} \prod_{j=1}^{k} X\left(\frac{1}{2}-\alpha_{j}\right)^{-\frac{1}{2}} \sum_{\epsilon_{j}= \pm 1} \prod_{j=1}^{k} \epsilon_{j} X\left(\frac{1}{2}+\epsilon_{j} \alpha_{j}\right)^{-\frac{1}{2}} \\
\times \prod_{1 \leq i<j \leq k} \zeta\left(1+\epsilon_{i} \alpha_{i}+\epsilon_{j} \alpha_{j}\right) A_{k}\left(\epsilon_{1} \alpha_{1}, \ldots, \epsilon_{k} \alpha_{k}\right)\left(1+O(n q)^{-\frac{1}{2}+\varepsilon}\right) .
\end{array}
\end{aligned}
$$


The above formulae can be written as contour integrals using Lemma 2.5.2, giving expressions analogous to those in Conjecture 1.5.5. In particular, expressing (4.5.11) as a contour integral and then letting $\alpha_{j} \rightarrow 0$ gives Conjecture 1.5.5.

\section{Numerical CALCUlations}

We compare our conjectures with some numerical calculations. The agreement is very good. These calculations involve numerically approximating the coefficients in the conjectured formulae, and numerically evaluating the mean value. Both of those calculations are interesting and we will give more details and examples in a subsequent paper.

5.1. Unitary: Riemann zeta-function. The coefficients of $P_{2}(x)$ in Conjecture 1.5.1 can be written explicitly in terms of known constants. When $k=2$ the function $G\left(\alpha_{1}, \alpha_{2}, \alpha_{3}, \alpha_{4}\right)$ that appears in Conjecture 1.5.1 equals

$$
\zeta\left(2+\alpha_{1}+\alpha_{2}-\alpha_{3}-\alpha_{4}\right)^{-1} \prod_{i, j=1}^{2} \zeta\left(1+\alpha_{i}-\alpha_{k+j}\right)
$$

which is given in Section 2.2

But

$$
\zeta(1+s)=s^{-1}+\gamma-\gamma_{1} s+\frac{\gamma_{2}}{2 !} s^{2}-\frac{\gamma_{3}}{3 !} s^{3}+\ldots
$$

and

$$
\zeta(2+s)^{-1}=\frac{6}{\pi^{2}}-\frac{36 \zeta^{\prime}(2)}{\pi^{4}} s+\frac{-3 \pi^{2} \zeta^{\prime \prime}(2)+36 \zeta^{\prime}(2)^{2}}{\pi^{6}} s^{2}+\ldots
$$

In the latter, the terms up to $s^{4}$ were evaluated using Maple. Computing the residue in Conjecture 1.5.1 gives

$$
\begin{aligned}
& P_{2}(x)=\frac{1}{2 \pi^{2}} x^{4}+\frac{8}{\pi^{4}}\left(\gamma \pi^{2}-3 \zeta^{\prime}(2)\right) x^{3} \\
& +\frac{6}{\pi^{6}}\left(-48 \gamma \zeta^{\prime}(2) \pi^{2}-12 \zeta^{\prime \prime}(2) \pi^{2}+7 \gamma^{2} \pi^{4}+144 \zeta^{\prime}(2)^{2}-2 \gamma_{1} \pi^{4}\right) x^{2} \\
& +\frac{12}{\pi^{8}}\left(6 \gamma^{3} \pi^{6}-84 \gamma^{2} \zeta^{\prime}(2) \pi^{4}+24 \gamma_{1} \zeta^{\prime}(2) \pi^{4}-1728 \zeta^{\prime}(2)^{3}+576 \gamma \zeta^{\prime}(2)^{2} \pi^{2}\right. \\
& \left.+288 \zeta^{\prime}(2) \zeta^{\prime \prime}(2) \pi^{2}-8 \zeta^{\prime \prime \prime}(2) \pi^{4}-10 \gamma_{1} \gamma \pi^{6}-\gamma_{2} \pi^{6}-48 \gamma \zeta^{\prime \prime}(2) \pi^{4}\right) x \\
& +\frac{4}{\pi^{10}(}-12 \zeta^{\prime \prime \prime \prime}(2) \pi^{6}+36 \gamma_{2} \zeta^{\prime}(2) \pi^{6}+9 \gamma^{4} \pi^{8}+21 \gamma_{1}^{2} \pi^{8}+432 \zeta^{\prime \prime}(2)^{2} \pi^{4} \\
& +3456 \gamma \zeta^{\prime}(2) \zeta^{\prime \prime}(2) \pi^{4}+3024 \gamma^{2} \zeta^{\prime}(2)^{2} \pi^{4}-36 \gamma^{2} \gamma_{1} \pi^{8}-252 \gamma^{2} \zeta^{\prime \prime}(2) \pi^{6} \\
& +3 \gamma \gamma_{2} \pi^{8}+72 \gamma_{1} \zeta^{\prime \prime}(2) \pi^{6}+360 \gamma_{1} \gamma \zeta^{\prime}(2) \pi^{6}-216 \gamma^{3} \zeta^{\prime}(2) \pi^{6} \\
& \quad-864 \gamma_{1} \zeta^{\prime}(2)^{2} \pi^{4}+5 \gamma_{3} \pi^{8}+576 \zeta^{\prime}(2) \zeta^{\prime \prime \prime}(2) \pi^{4}-20736 \gamma \zeta^{\prime}(2)^{3} \pi^{2} \\
& \left.\quad-15552 \zeta^{\prime \prime}(2) \zeta^{\prime}(2)^{2} \pi^{2}-96 \gamma \zeta^{\prime \prime \prime}(2) \pi^{6}+62208 \zeta^{\prime}(2)^{4}\right),
\end{aligned}
$$


in agreement with a result implied in the work of Heath-Brown[H-B1] (see [C] where, using [H-B1], the same polynomial is worked out, although there are some slight errors). Numerically,

$$
\begin{aligned}
P_{2}(x)= & 0.0506605918211688857219397316048638 x^{4} \\
& +0.69886988487897996984709628427658502 x^{3} \\
& +2.425962198846682004756575310160663 x^{2} \\
& +3.227907964901254764380689851274668 x \\
& +1.312424385961669226168440066229978
\end{aligned}
$$

There are several ways that one can numerically compute the coefficients of $P_{3}(x)$, and these will be described in a future paper. We found

$$
\begin{aligned}
P_{3}(x)= & 0.000005708527034652788398376841445252313 x^{9} \\
& +0.00040502133088411440331215332025984 x^{8} \\
& +0.011072455215246998350410400826667 x^{7} \\
& +0.14840073080150272680851401518774 x^{6} \\
& +1.0459251779054883439385323798059 x^{5} \\
& +3.984385094823534724747964073429 x^{4} \\
& +8.60731914578120675614834763629 x^{3}+10.274330830703446134183009522 x^{2} \\
& +6.59391302064975810465713392 x+0.9165155076378930590178543 .
\end{aligned}
$$

One notices that the leading coefficient is much smaller than the lower order coefficients, which means that, in numerical calculations, the lower order terms will contribute significantly. One might suppose that the coefficients of $P_{k}(x)$ are always positive. Unfortunately, while this is true for $P_{1}, \ldots, P_{4}$, by $k=5$, negative coefficients begin to appear (see Table 5.1.1).

Table 5.1 .2 depicts

$$
\int_{C}^{D}\left|\zeta\left(\frac{1}{2}+i t\right)\right|^{6} d t
$$

as compared to

$$
\int_{C}^{D} P_{3}(\log (t / 2 \pi)) d t
$$

along with their ratio, for various blocks $[C, D]$ of length 50000 , as well as a larger block of length 2,350,000. The data agree with our conjecture and are consistent with a remainder of size $|D-C|^{\frac{1}{2}} D^{\varepsilon}$.

One can also look at smoothed moments, for example,

$$
\int_{0}^{\infty}\left|\zeta\left(\frac{1}{2}+i t\right)\right|^{2 k} \exp (-t / T) d t
$$

as compared to

$$
\int_{0}^{\infty} P_{k}(\log (t / 2 \pi)) \exp (-t / T) d t
$$


Table 5.1.3 compares these with $T=10000$, for $k=4,3,2,1$.

For $k=3,4$ the data agrees to roughly half the decimal places. This supports our conjecture that the error term in the conjectured mean values is $O\left(T^{\frac{1}{2}+\varepsilon}\right)$. For $k=1$ the numerics suggest Corollary 1.6.3.

5.2. Symplectic: quadratic Dirichlet $L$-functions. We have computed the polynomials $Q_{k}$ of Conjecture 1.5.3 for $k=1,2, \ldots, 8$, separately for $d<0$ and $d>0$. Table 5.2.1 lists these polynomials for $d<0$, while in Table 5.2.2 we consider $d>0$. Again notice the small size of the leading coefficients.

Table 5.2 .3 compares, for $d<0$, conjectured moments for $k=1, \ldots, 8$ against numerically computed moments,

$$
\sum_{d<0}^{*} L\left(\frac{1}{2}, \chi_{d}\right)^{k} g(|d|)
$$

versus

$$
\sum_{d<0}^{*} Q_{k}(\log |d|) g(|d|)
$$

where $g$ is the smooth test function

$$
g(t)= \begin{cases}1, & \text { if } 0 \leq t<850000 \\ \exp \left(1-\frac{1}{\left.1-\frac{(t-850000)^{2}}{(150000)^{2}}\right),}\right. & \text { if } 850000 \leq t \leq 1000000 \\ 0, & \text { if } 1000000<t\end{cases}
$$

Table 5.2 .4 compares the same quantities, but for $d>0$.

Figure 1 depicts, for $k=1, \ldots, 8$ and $X=10000,20000, \ldots, 10^{7}$,

$$
\sum_{-X<d<0}^{*} L\left(\frac{1}{2}, \chi_{d}\right)^{k}
$$

divided by

$$
\sum_{-X<d<0}^{*} Q_{k}(\log |d|) .
$$

One sees the graphs fluctuating above and below one. Interestingly, the graphs have a similar shape as $k$ varies. This is explained by the fact that large values of $L\left(1 / 2, \chi_{d}\right)$ tend to skew the moments, and this gets amplified as $k$ increases.

Figure 2 depicts the same but for $0<d \leq X$.

The values $L\left(\frac{1}{2}, \chi_{d}\right)$ were computed using a smoothed form of the approximate functional equation which expresses the $L$-function in terms of the incomplete Gamma-function (see for example $[\mathrm{L}]$ ).

5.3. Orthogonal: twists of a $G L(2) L$-function. Let

$$
L_{11}(s)=\sum_{n=1}^{\infty} \frac{a_{n}}{n^{\frac{1}{2}+s}}
$$


be the $L$-function of conductor 11 of the elliptic curve

$$
y^{2}+y=x^{3}-x^{2} .
$$

The coefficients $a_{n}$ are obtained from the cusp form of weight two and level 11 given by

$$
\sum_{n=1}^{\infty} a_{n} q^{n}=q \prod_{n=1}^{\infty}\left(1-q^{n}\right)^{2}\left(1-q^{11 n}\right)^{2}
$$

Expanding the right side using Euler's pentagonal theorem provides an efficient means to compute the $a_{n}$ 's.

$L_{11}(s)$ satisfies an even functional equation (i.e. $\left.\varepsilon=+1\right)$ :

$$
\left(\frac{11^{\frac{1}{2}}}{2 \pi}\right)^{s} \Gamma\left(s+\frac{1}{2}\right) L_{11}(s)=\left(\frac{11^{\frac{1}{2}}}{2 \pi}\right)^{1-s} \Gamma\left(\frac{3}{2}-s\right) L_{11}(1-s),
$$

and may be written as a product over primes

$$
L_{11}(s)=\frac{1}{1-11^{-s-\frac{1}{2}}} \prod_{p \neq 11} \frac{1}{1-a_{p} p^{-s-\frac{1}{2}}+p^{-2 s}} .
$$

Consider now quadratic twists of $L_{11}(s)$,

$$
L_{11}\left(s, \chi_{d}\right)=\sum_{n=1}^{\infty} \frac{a_{n}}{n^{\frac{1}{2}+s}} \chi_{d}(n) .
$$

with $(d, 11)=1 . L_{11}\left(s, \chi_{d}\right)$ satisfies the functional equation

$$
L_{11}\left(s, \chi_{d}\right)=\chi_{d}(-11) \frac{\Gamma\left(\frac{3}{2}-s\right)}{\Gamma\left(s+\frac{1}{2}\right)}\left(\frac{2 \pi}{11^{\frac{1}{2}}}\right)^{2 s-1}|d|^{2\left(\frac{1}{2}-s\right)} L_{11}\left(1-s, \chi_{d}\right) .
$$

We wish to look at moments of $L_{11}\left(\frac{1}{2}, \chi_{d}\right)$ but only for those $L\left(s, \chi_{d}\right)$ that have an even functional equation, i.e. $\chi_{d}(-11)=1$. We further only look at $d<0$ since in that case a theorem of Kohnen and Zagier [KZ] enables us to easily gather numerical data for $L_{11}\left(\frac{1}{2}, \chi_{d}\right)$ with which to check our conjecture.

When $d<0, \chi_{d}(-1)=-1$, hence, in order to have even functional equation, we require $\chi_{d}(11)=-1$, i.e. $d=2,6,7,8,10 \bmod 11$. Conjectured formula (4.4.22) combined with Lemma 2.5 .2 gives an estimate for the sum over fundamental discriminants

$$
\sum_{\substack{-D<d<0 \\ d=2,6,7,8,10 \bmod 11}}^{*} L_{11}\left(\frac{1}{2}, \chi_{d}\right)^{k}=\sum_{\substack{-D<d<0 \\ d=2,6,7,8,10 \bmod 11}}^{*} \Upsilon_{k}(\log |d|)+O\left(D^{\frac{1}{2}+\varepsilon}\right)
$$

where, as in Section 4.4, $\Upsilon_{k}$ is the polynomial of degree $k(k-1) / 2$ given by the $k$-fold residue

$$
\Upsilon_{k}(x)=\frac{(-1)^{k(k-1) / 2} 2^{k}}{k !} \frac{1}{(2 \pi i)^{k}} \oint \cdots \oint \frac{R_{11}\left(z_{1}, \ldots, z_{k}\right) \Delta\left(z_{1}^{2}, \ldots, z_{k}^{2}\right)^{2}}{\prod_{j=1}^{k} z_{j}^{2 k-1}} e^{x \sum_{j=1}^{k} z_{j}} d z_{1} \ldots d z_{k},
$$


where

$$
R_{11}\left(z_{1}, \ldots, z_{k}\right)=A_{k}\left(z_{1}, \ldots, z_{k}\right) \prod_{j=1}^{k}\left(\frac{\Gamma\left(1+z_{j}\right)}{\Gamma\left(1-z_{j}\right)}\left(\frac{11}{4 \pi^{2}}\right)^{z_{j}}\right)^{\frac{1}{2}} \prod_{1 \leq i<j \leq k} \zeta\left(1+z_{i}+z_{j}\right)
$$

and $A_{k}$ is the Euler product which is absolutely convergent for $\sum_{j=1}^{k}\left|z_{j}\right|<\frac{1}{2}$,

$$
A_{k}\left(z_{1}, \ldots, z_{k}\right)=\prod_{p} R_{11, p}\left(z_{1}, \ldots, z_{k}\right) \prod_{1 \leq i<j \leq k}\left(1-\frac{1}{p^{1+z_{i}+z_{j}}}\right)
$$

with, for $p \neq 11$,

$$
R_{11, p}=\left(1+\frac{1}{p}\right)^{-1}\left(\frac{1}{p}+\frac{1}{2}\left(\prod_{j=1}^{k} \frac{1}{1-a_{p} p^{-1-z_{j}}+p^{-1-2 z_{j}}}+\prod_{j=1}^{k} \frac{1}{1+a_{p} p^{-1-z_{j}}+p^{-1-2 z_{j}}}\right)\right)
$$

and

$$
R_{11,11}=\prod_{j=1}^{k} \frac{1}{1+11^{-1-z_{j}}}
$$

Numerically, it is more challenging to compute the polynomials $\Upsilon_{k}$. First, using

$$
\prod_{1 \leq i<j \leq k} \zeta\left(1+z_{i}+z_{j}\right)
$$

to estimate the sum over primes of (4.4.17) makes a poor approximation and one would do better to use the Rankin-Selberg convolution $L$-function of $L_{11}(s)$ with itself. However, it is simpler to work with $\zeta$, and we thus computed the first 4 moment polynomials of $L_{11}\left(\frac{1}{2}, \chi_{d}\right)$ but to low accuracy. The coefficients of these polynomials are given to 2-5 decimal place accuracy in Table 5.3.1.

In Table 5.3 .2 we compare moments computed numerically with moments estimated by our conjecture. The two agree to within the accuracy we have for the moment polynomial coefficients. We believe that if one were to compute the coefficients to higher accuracy, one would see an even better agreement with the data.

While one can compute $L_{11}\left(\frac{1}{2}, \chi_{d}\right)$ using standard techniques (see Coh]), in our case we can exploit a theorem of Kohnen and Zagier [KZ] which relates $L_{11}\left(\frac{1}{2}, \chi_{d}\right)$, for fundamental discriminants $d<0, d \equiv 2,6,7,8,10 \bmod 11$, to the coefficients $c_{11}(|d|)$ of a weight $3 / 2$ modular form

$$
L_{11}\left(\frac{1}{2}, \chi_{d}\right)=\kappa_{11} c_{11}(|d|)^{2} / \sqrt{d}
$$

where $\kappa_{11}$ is a constant. The weight $3 / 2$ form in question was determined by RodriguezVillegas (private communication)

$$
\begin{aligned}
\sum_{n=1}^{\infty} c_{11}(n) q^{n} & =\left(\theta_{1}(q)-\theta_{2}(q)\right) / 2 \\
& =-q^{3}+q^{4}+q^{11}+q^{12}-q^{15}-2 q^{16}-q^{20} \ldots
\end{aligned}
$$


where

$$
\theta_{1}(q)=\sum_{\substack{(x, y, z) \in \mathbb{Z}^{3} \\ x \equiv y \bmod 2}} q^{x^{2}+11 y^{2}+11 z^{2}}=1+2 q^{4}+2 q^{11}+4 q^{12}+4 q^{15}+2 q^{16}+4 q^{20} \ldots
$$

and

$$
\theta_{2}(q)=\sum_{\substack{(x, y, z) \in \mathbb{Z}^{3} \\ x \equiv y \bmod 3 \\ y \equiv z \bmod 2}} q^{\left(x^{2}+11 y^{2}+33 z^{2}\right) / 3}=1+2 q^{3}+2 q^{12}+6 q^{15}+6 q^{16}+6 q^{20} \ldots
$$

This was used to compute the $c_{11}(|d|)$ 's for $d<85,000,000$.

Evaluating the left side of (5.3.8) in a more traditional manner for $d=-3$, and comparing with the right side, we determined

$$
\kappa_{11}=2.917633233876991 .
$$

\section{REFERENCES}

[A] F. V. Atkinson, The mean value of the zeta-function on the critical line, Proc. London Math. Soc. (2) 471941 pp. 174-200.

[BC] A. Borel and W. Casselman, eds, Automorphic Forms, Representations, and $L$-functions, AMS Proceedings of Symposia in Pure Mathematics 33, Amer. Math. Soc., Providence, RI 1979.

[BF] E.L. Basor and P.J. Forrester, Formulas for the evaluation of Toeplitz determinants with rational generating functions, Math. Nachr. 1701994 pp. 5-18.

[BH] E. Brézin and S. Hikami, Characteristic polynomials of random matrices, Comm. Math. Phys. 214 2000 pp. 111-135.

[Bu] D. Bump, Automorphic forms and representations. Cambridge Studies in Advanced Mathematics, 55, Cambridge University Press addr Cambridge 1997.

[Coh] H. Cohen, A Course in computational algebraic number theory, Springer Verlag addr Berlin 1995.

[C] J. B. Conrey, A note on the fourth power moment of the Riemann zeta-function, in Analytic number theory, (Allerton Park, IL, 1995), Progr. Math. 138 Birkhäuser Boston, MA, 1996.

$[\mathrm{CF}] \quad$ J. B. Conrey and D. W. Farmer, Mean values of L-functions and symmetry, Internat. Math. Res. Notices 200017 pp. 883-908.

[CFKRS] J. B. Conrey, D. W. Farmer, J. P. Keating, M. O. Rubinstein, and N. C. Snaith, Autocorrelation of random matrix polynomials, Commun. Math. Phys 237 (2003) 3, pp. 365-395.

[CG1] J. B. Conrey and A. Ghosh, Mean values of the Riemann zeta-function, Mathematika 311984 pp. 159-161.

[CG2] J. B. Conrey and A. Ghosh, A conjecture for the sixth power moment of the Riemann zeta-function, Int. Math. Res. Not. 151998 pp. 775-780.

[CG3] J. B. Conrey and A. Ghosh, On the Selberg class of Dirichlet series: small degrees, Duke Math. J. 721993 pp. 673-693.

[CGo] J. B. Conrey and S. M. Gonek, High moments of the Riemann zeta-function, Duke Math. Jour. 2001107 pp. 577-604.

[Dav] H. Davenport, Multiplicative Number Theory, GTM 74 addr Springer-Verlag, New York, NY 2000.

[DGH] A. Diaconu, D. Goldfeld, and J. Hoffstein, Multiple Dirichlet series and moments of zeta- and $L$-functions, Compositio Math. 139 (2003), no. 3, 297-360. ArXiv: math.nt/0110092

[D] W. Duke, The critical order of vanishing of automorphic L-functions with large level, Invent. Math. 1191995 pp. 165-174.

[DFI] W. Duke, J. B. Friedlander, and H. Iwaniec, Bounds for automorphic L-functions. II, Invent. Math. 1151994 pp. 219-239.

[GV] D. Goldfeld and C. Viola, Mean values of L-functions associated to elliptic, Fermat and other curves at the centre of the critical strip, J. Number Theory 11 1979, S. Chowla Anniversary Issue pp. 305-320. 
[Go] A. Good, The square mean of Dirichlet series associated with cusp forms, Mathematika 291983 pp. 278-295.

[HI] J.L. Hafner and A. Ivić, On the mean-square of the Riemann zeta-function on the critical line, 1989 J. Number Theory pp. 151-191 32.

[HL] G. H. Hardy and J. E. Littlewood, Contributions to the theory of the Riemann zeta-function and the theory of the distribution of primes, 1918 Acta Mathematica pp. 119 - 19641.

[H-B1] D. R. Heath-Brown, The fourth power moment of the Riemann zeta-function, Proc. London Math. Soc. (3) 197938 pp. $385-422$.

[H-B2] D. R. Heath-Brown, An asymptotic series for the mean value of Dirichlet L-functions, Comment. Math. Helv. 561981 pp. 148-161.

[H-B3] D. R. Heath-Brown, The fourth power mean of Dirichlet's L-functions, Analysis 11981 pp. 25-32.

[I] A. E. Ingham, Mean-value theorems in the theory of the Riemann zeta-function, Proceedings of the London Mathematical Society (92) 192627 pp. 273-300.

[Iv1] A. Ivić, On the fourth moment of the Riemann zeta function, Publs. Inst. Math. (Belgrade) 57 (71) 199527 pp. 101-110.

[Iv2] A. Ivić, Moments of Hecke series at central points, Functiones et Approximatio 30200227 pp. 49-82.

[Iw1] H. Iwaniec, Topics in classical automorphic forms. Graduate Studies in Mathematics, 17, American Mathematical Society addr Providence, RI 1997.

[Iw2] H. Iwaniec, Spectral methods of automorphic forms, 2nd ed. Graduate Studies in Mathematics, 53, American Mathematical Society, Providence, RI 1997.

[IK] H. Iwaniec and E. Kowalksi, Analytic Number Theory. American Mathematical Society, Providence, RI 2004.

[JS] H. Jacquet and Shalika, A nonvanishing theorem for zeta functions on $G L_{n}$, Invent. Math. 38 1976 pp. 1-16.

$[\mathrm{J}] \quad$ M. Jutila, On the mean value of $L\left(\frac{1}{2}, \chi\right)$ for real characters, Analysis 11981 149-161.

[KSa] N. M. Katz and P. Sarnak, Random matrices, Frobenius eigenvalues, and monodromy, AMS Colloquium Publications, 45 addr AMS, Providence, RI 1999.

[KS1] J. P. Keating and N. C. Snaith, Random matrix theory and $\zeta\left(\frac{1}{2}+i t\right)$, Comm. Math. Phys. 214 2000 pp. 57-89.

[KS2] J. P. Keating and N. C. Snaith, Random matrix theory and L-functions at $s=\frac{1}{2}$, Comm. Math. Phys. 2142000 pp. 91-110.

[Ko] H. Kober, Eine Mittelwortformel der Riemannschen Zetafunktion, Compositio Math. 31936 pp. 174-189.

[KZ] Kohnen, W. and Zagier, D., Values of L-series of modular forms at the center of the critical strip, Invent. Math. 641981 pp. 175-198.

[KMV] E. Kowalski, P. Michel, and J. M. VanderKam, Mollification of the fourth moment of automorphic L-functions and arithmetic applications, Invent. Math. 1422000 pp. 95-151.

[L] A. F. Lavrik, Approximate functional equation for Dirichlet Functions, Izv. Akad. Nauk SSSR 32 1968 pp. $134-185$.

[LM] N. Levinson and H. Montgomery, Zeros of the derivatives of the Riemann zeta-function, Acta Math. 1331974 pp. 49-65.

[Meh] M. Mehta, Random Matrix Theory, Academic Press addr Boston 1991.

[Mon] H.L. Montgomery, The pair correlation of zeros of the Riemann zeta-function, Proc. Symp. Pure Math. 241973 pp. 181-93.

[Mot] Y. Motohashi, Spectral theory of the Riemann zeta-function, Cambridge Tracts in Mathematics 127 Cambridge University Press addr Cambridge 1997.

[Mur] M.R. Murty, Selberg's conjectures and Artin L-functions, Bull. Amer. Math. Soc. (N.S.) 311994 pp. $1-14$.

[Odl] A. Odlyzko, The $10^{20}$ th zero of the Riemann zeta-function and 70 million of its neighbors., Preprint 1989.

[OS] A. Özlük and C. Snyder, Small zeros of quadratic L-functions, Bull. Austral. Math. Soc. 471993 pp. 307-319.

[Rio] J. Riordan, Combinatorial Identities, John Wiley \& Sons, Inc addr New York 1968 
[Rub] M. O. Rubinstein, Evidence for a spectral interpretation of the zeros of L-functions, 1998 thesis, Princeton University.

[RS] Z. Rudnick and P. Sarnak, Zeros of principal L-functions and random matrix theory, Duke Math. J. 811996 pp. 269-322.

[S] A. Selberg, Old and new conjectures and results about a class of Dirichlet series, Collected Papers, Vol. 2 Springer-Verlag addr Berlin 1991.

[So] K. Soundararajan, Non-vanishing of quadratic Dirichlet L-functions at $s=\frac{1}{2}$, Ann. of Math. (2) 1522000 pp. 447-488.

[T] E. C. Titchmarsh, The theory of the Riemann zeta-function. Second edition. Edited and with a preface by D. R. Heath-Brown, The Clarendon Press, Oxford University Press addr New York 1986.

[Z] Q. Zhang, On the cubic moment of quadratic Dirichlet L-functions, preprint.

American Institute of Mathematics

360 Portage Ave.

Palo Alto, CA 94306

Department of Mathematics

OKlahoma State University

STILLWATER, OK 74078-0613

SChOOL OF Mathematics

UNIVERSITY OF BRISTOL

Clifton, Bristol

BS8 1TW

United KingDom

Pure Mathematics

UniVERSity of WATERLOO

WATERLOo, Ontario

N2L 3G1

CANADA 


\begin{tabular}{|c|c|c|c|c|}
\hline$r$ & $c_{r}(4)$ & $c_{r}(5)$ & $c_{r}(6)$ & $c_{r}(7)$ \\
\hline 0 & $.24650183919342276 \mathrm{e}-12$ & $.141600102062273 \mathrm{e}-23$ & $.512947340914913 \mathrm{e}-39$ & $.658228478760010 \mathrm{e}-59$ \\
\hline 1 & $.54501405731171861 \mathrm{e}-10$ & $.738041275649445 \mathrm{e}-21$ & $.530673280992642 \mathrm{e}-36$ & $.120414305554514 \mathrm{e}-55$ \\
\hline 2 & $.52877296347912035 \mathrm{e}-8$ & $.177977962351965 \mathrm{e}-18$ & $.260792077114835 \mathrm{e}-33$ & $.106213557174925 \mathrm{e}-52$ \\
\hline 3 & $.29641143179993979 \mathrm{e}-6$ & $.263588660966072 \mathrm{e}-16$ & $.810161321577902 \mathrm{e}-31$ & $.601726537601586 \mathrm{e}-50$ \\
\hline 4 & $.1064595006812847 \mathrm{e}-4$ & $.268405453499975 \mathrm{e}-14$ & $.178612973800931 \mathrm{e}-28$ & $.246062876732400 \mathrm{e}-47$ \\
\hline 5 & $.25702983342426343 \mathrm{e}-3$ & $.199364130924990 \mathrm{e}-12$ & $.297431671086361 \mathrm{e}-26$ & $.773901216652114 \mathrm{e}-45$ \\
\hline 6 & $.42639216163116947 \mathrm{e}-2$ & $.111848551249336 \mathrm{e}-10$ & $.388770829115587 \mathrm{e}-24$ & $.194786494949524 \mathrm{e}-42$ \\
\hline 7 & $.48941424514215989 \mathrm{e}-1$ & $.484279755304480 \mathrm{e}-9$ & $.409224261406863 \mathrm{e}-22$ & $.403076849263637 \mathrm{e}-40$ \\
\hline 8 & .38785267 & $.16398013 \mathrm{e}-7$ & $.35314664 \mathrm{e}-20$ & $.69917763 \mathrm{e}-38$ \\
\hline 9 & 2.1091338 & $.43749351 \mathrm{e}-6$ & $.25306377 \mathrm{e}-18$ & $.1031402 \mathrm{e}-35$ \\
\hline 10 & 7.8325356 & $.92263335 \mathrm{e}-5$ & $.15198191 \mathrm{e}-16$ & $.13082869 \mathrm{e}-33$ \\
\hline 11 & 19.828068 & .00015376778 & $.77001514 \mathrm{e}-15$ & $.14392681 \mathrm{e}-31$ \\
\hline 12 & 33.888932 & .0020190278 & $.3306121 \mathrm{e}-13$ & $.13825312 \mathrm{e}-29$ \\
\hline 13 & 38.203306 & .020772707 & $.12064042 \mathrm{e}-11$ & $.11657759 \mathrm{e}-27$ \\
\hline 14 & 25.604415 & .16625059 & $.37467193 \mathrm{e}-10$ & $.86652477 \mathrm{e}-26$ \\
\hline 15 & 10.618974 & 1.0264668 & $.99056943 \mathrm{e}-9$ & $.56962227 \mathrm{e}-24$ \\
\hline 16 & .708941 & 4.8485893 & $.22273886 \mathrm{e}-7$ & $.33197649 \mathrm{e}-22$ \\
\hline 17 & & 17.390876 & $.42513729 \mathrm{e}-6$ & $.1718397 \mathrm{e}-20$ \\
\hline 18 & & 47.040877 & $.68674336 \mathrm{e}-5$ & $.79096789 \mathrm{e}-19$ \\
\hline 19 & & 95.116618 & $.9351583 \mathrm{e}-4$ & $.32396929 \mathrm{e}-17$ \\
\hline 20 & & 141.44446 & .0010683164 & $.11809579 \mathrm{e}-15$ \\
\hline 21 & & 149.35697 & .010180702 & $.3830227 \mathrm{e}-14$ \\
\hline 22 & & 105.88716 & .080418679 & $.11044706 \mathrm{e}-12$ \\
\hline 23 & & 44.1356 & .52296142 & $.28282258 \mathrm{e}-11$ \\
\hline 24 & & 20.108 & 2.7802018 & $.64210662 \mathrm{e}-10$ \\
\hline 25 & & -1.27 & 12.001114 & $.12898756 \mathrm{e}-8$ \\
\hline 26 & & & 41.796708 & $.22869667 \mathrm{e}-7$ \\
\hline 27 & & & 116.72309 & $.35683995 \mathrm{e}-6$ \\
\hline 28 & & & 259.39898 & $.48834071 \mathrm{e}-5$ \\
\hline 29 & & & 452.491 & $.58391045 \mathrm{e}-4$ \\
\hline 30 & & & 601.17 & .00060742037 \\
\hline 31 & & & 573.54 & .0054716438 \\
\hline 32 & & & 374.8 & .042465904 \\
\hline 33 & & & 246.5 & .28245494 \\
\hline 34 & & & 248 & 1.6013331 \\
\hline 35 & & & $1.6 \mathrm{e}+02 ?$ & 7.6966995 \\
\hline 36 & & & $-4 . e+01 ?$ & 31.20352 \\
\hline 37 & & & & 106.19714 \\
\hline 38 & & & & 301.91363 \\
\hline 39 & & & & 711.742 \\
\hline 40 & & & & 1370.10 \\
\hline 41 & & & & 2083 . \\
\hline 42 & & & & 2356 . \\
\hline 43 & & & & $1.9 \mathrm{e}+03$ \\
\hline 44 & & & & $1.8 \mathrm{e}+03$ \\
\hline 45 & & & & $3 . e+03$ \\
\hline 46 & & & & $3 . e+03$ \\
\hline 47 & & & & $8 . e+01 ?$ \\
\hline 48 & & & & $-1 . e+03 ?$ \\
\hline 49 & & & & $-2 . e+02 ?$ \\
\hline
\end{tabular}

TABLE 5.1.1. Coefficients of $P_{k}(x)=c_{0}(k) x^{k^{2}}+c_{1}(k) x^{k^{2}-1}+\cdots+c_{k^{2}}(k)$, for $k=4,5,6,7$. Notice the relatively small size of $c_{0}(k)$. We believe the coefficients to be correct to the number of places listed, except in the cases indicated by question marks, where the numerics have not quite stabilized. Two different methods were used to compute the coefficients. The former, for $0 \leq r \leq 7$, gave us higher precision but was less efficient, while the latter for $r \leq 49$, was more efficient but required using less precision. 


\begin{tabular}{|c|c|c|c|}
\hline$[C, D]$ & conjecture (5.1.8) & reality (5.1.7) & ratio \\
\hline$[0,50000]$ & 7236872972.7 & 7231005642.3 & .999189 \\
{$[50000,100000]$} & 15696470555.3 & 15723919113.6 & 1.001749 \\
{$[100000,150000]$} & 21568672884.1 & 21536840937.9 & .998524 \\
{$[150000,200000]$} & 26381397608.2 & 26246250354.1 & .994877 \\
{$[200000,250000]$} & 30556177136.5 & 30692229217.8 & 1.004453 \\
{$[250000,300000]$} & 34290291841.0 & 34414329738.9 & 1.003617 \\
{$[300000,350000]$} & 37695829854.3 & 37683495193.0 & .999673 \\
{$[350000,400000]$} & 40843941365.7 & 40566252008.5 & .993201 \\
{$[400000,450000]$} & 43783216365.2 & 43907511751.1 & 1.002839 \\
{$[450000,500000]$} & 46548617846.7 & 46531247056.9 & .999627 \\
{$[500000,550000]$} & 49166313161.9 & 49136264678.2 & .999389 \\
{$[550000,600000]$} & 51656498739.2 & 51744796875.0 & 1.001709 \\
{$[600000,650000]$} & 54035153255.1 & 53962410634.2 & .998654 \\
{$[650000,700000]$} & 56315178564.8 & 56541799179.3 & 1.004024 \\
{$[700000,750000]$} & 58507171421.6 & 58365383245.2 & .997577 \\
{$[750000,800000]$} & 60619962488.2 & 60870809317.1 & 1.004138 \\
{$[800000,850000]$} & 62661003164.6 & 62765220708.6 & 1.001663 \\
{$[850000,900000]$} & 64636649728.0 & 64227164326.1 & .993665 \\
{$[900000,950000]$} & 66552376294.2 & 65994874052.2 & .991623 \\
{$[950000,1000000]$} & 68412937271.4 & 68961125079.8 & 1.008013 \\
{$[1000000,1050000]$} & 70222493232.7 & 70233393177.0 & 1.000155 \\
{$[1050000,1100000]$} & 71984709805.4 & 72919426905.7 & 1.012985 \\
{$[1100000,1150000]$} & 73702836332.4 & 72567024812.4 & .984589 \\
{$[1150000,1200000]$} & 75379769148.4 & 76267763314.7 & 1.011780 \\
{$[1200000,1250000]$} & 77018102997.5 & 76750297112.6 & .996523 \\
{$[1250000,1300000]$} & 78620173202.6 & 78315210623.9 & .996121 \\
{$[1300000,1350000]$} & 80188090542.5 & 80320710380.9 & 1.001654 \\
{$[1350000,1400000]$} & 81723770322.2 & 80767881132.6 & .988303 \\
{$[1400000,1450000]$} & 83228956776.3 & 83782957374.3 & 1.006656 \\
{$[0,2350000]$} & 3317437762612.4 & 3317496016044.9 & 1.000017 \\
\hline
\end{tabular}

TABLE 5.1.2. Sixth moment of $\zeta$ versus Conjecture 1.5.1], The 'reality' column, i.e. integrals involving $\zeta$, were computed using Mathematica.

\begin{tabular}{|c|c|c|c|c|}
\hline$k$ & $(5.1 .9)$ & (5.1.10) & difference & $\begin{array}{c}\text { relative } \\
\text { difference }\end{array}$ \\
\hline 1 & 79499.9312635 & 79496.7897047 & 3.14156 & $3.952 \times 10^{-5}$ \\
2 & 5088332.55512 & 5088336.43654 & -3.8814 & $-7.628 \times 10^{-7}$ \\
3 & 708967359.4 & 708965694.5 & 1664.9 & $2.348 \times 10^{-6}$ \\
4 & 143638308513.0 & 143628911646.6 & 9396866.4 & $6.542 \times 10^{-5}$ \\
\hline
\end{tabular}

TABLE 5.1.3. Smoothed moment of $\zeta$ versus Conjecture 1.5.1. 


\begin{tabular}{|c|c|c|c|c|}
\hline$r$ & $d_{r}(1)$ & $d_{r}(2)$ & $d_{r}(3)$ & $d_{r}(4)$ \\
\hline 0 & .3522211004995828 & $.1238375103096 \mathrm{e}-1$ & $.1528376099282 \mathrm{e}-4$ & $.31582683324433 \mathrm{e}-9$ \\
\hline 1 & .61755003361406 & .18074683511868 & $.89682763979959 \mathrm{e}-3$ & $.50622013406082 \mathrm{e}-7$ \\
\hline 2 & & .3658991414081 & $.17014201759477 \mathrm{e}-1$ & $.32520704779144 \mathrm{e}-5$ \\
\hline 3 & & -.13989539029 & .10932818306819 & $.10650782552992 \mathrm{e}-3$ \\
\hline 4 & & & .13585569409025 & $.18657913487212 \mathrm{e}-2$ \\
\hline 5 & & & -.23295091113684 & $.16586741288851 \mathrm{e}-1$ \\
\hline 6 & & & .47353038377966 & $.59859999105052 \mathrm{e}-1$ \\
\hline 7 & & & & $.52311798496 \mathrm{e}-2$ \\
\hline 8 & & & & -.1097356195 \\
\hline 9 & & & & .55812532 \\
\hline 10 & & & & .19185945 \\
\hline$r$ & $d_{r}(5)$ & $d_{r}(6)$ & $d_{r}(7)$ & $d_{r}(8)$ \\
\hline 0 & $.671251761107 \mathrm{e}-16$ & $.1036004645427 \mathrm{e}-24$ & $.886492719 \mathrm{e}-36$ & $.337201 \mathrm{e}-49$ \\
\hline 1 & $.23412332535824 \mathrm{e}-13$ & $.67968140667178 \mathrm{e}-22$ & $.98944375081241 \mathrm{e}-33$ & $.59511917 \mathrm{e}-46$ \\
\hline 2 & $.35711692341033 \mathrm{e}-11$ & $.20378083365099 \mathrm{e}-19$ & $.51762930260135 \mathrm{e}-30$ & $.500204322 \mathrm{e}-43$ \\
\hline 3 & $.31271184907852 \mathrm{e}-9$ & $.36980514080794 \mathrm{e}-17$ & $.16867245856115 \mathrm{e}-27$ & $.2664702284 \mathrm{e}-40$ \\
\hline 4 & $.17346173129392 \mathrm{e}-7$ & $.45348387982697 \mathrm{e}-15$ & $.38372675160809 \mathrm{e}-25$ & $.1010164552 \mathrm{e}-37$ \\
\hline 5 & $.63429411057027 \mathrm{e}-6$ & $.39728668850800 \mathrm{e}-13$ & $.64746354773372 \mathrm{e}-23$ & $.29004988867 \mathrm{e}-35$ \\
\hline 6 & $.15410644373832 \mathrm{e}-4$ & $.2563279107877 \mathrm{e}-11$ & $.84021141030379 \mathrm{e}-21$ & $.65555882460 \mathrm{e}-33$ \\
\hline 7 & $.2441498848698 \mathrm{e}-3$ & $.12372292296 \mathrm{e}-9$ & $.85817644593981 \mathrm{e}-19$ & $.11966099802 \mathrm{e}-30$ \\
\hline 8 & .2390928284571e-2 & $.44915158297 \mathrm{e}-8$ & $.70024645896 \mathrm{e}-17$ & $.17958286298 \mathrm{e}-28$ \\
\hline 9 & $.127561073626 \mathrm{e}-1$ & $.1222154548 \mathrm{e}-6$ & $.4607034349989 \mathrm{e}-15$ & $.22443685425 \mathrm{e}-26$ \\
\hline 10 & $.24303820161 \mathrm{e}-1$ & $.2461203700 \mathrm{e}-5$ & $.2455973970377 \mathrm{e}-13$ & $.2357312577 \mathrm{e}-24$ \\
\hline 11 & $-.333141763 \mathrm{e}-1$ & $.3579140509 \mathrm{e}-4$ & $.106223013225 \mathrm{e}-11$ & $.20942850060 \mathrm{e}-22$ \\
\hline 12 & $.25775611 \mathrm{e}-1$ & $.3597968761 \mathrm{e}-3$ & $.3719625461492 \mathrm{e}-10$ & $.15805997923 \mathrm{e}-20$ \\
\hline 13 & .531596583 & $.230207769 \mathrm{e}-2$ & $.1048661496741 \mathrm{e}-8$ & $.10159435845 \mathrm{e}-18$ \\
\hline 14 & -.325832 & $.7699469185 \mathrm{e}-2$ & $.2357398870407 \mathrm{e}-7$ & $.55665248752 \mathrm{e}-17$ \\
\hline 15 & -1.34187 & $.4281359929 \mathrm{e}-2$ & $.416315210727 \mathrm{e}-6$ & $.25985097519 \mathrm{e}-15$ \\
\hline 16 & & $-.2312387714 \mathrm{e}-1$ & $.564739434674 \mathrm{e}-5$ & $.103134457 \mathrm{e}-13$ \\
\hline 17 & & .109503 & $.56831273239 \mathrm{e}-4$ & $.346778002 \mathrm{e}-12$ \\
\hline 18 & & .2900464 & $.40016131254 \mathrm{e}-3$ & $.982481680 \mathrm{e}-11$ \\
\hline 19 & & -.9016 & $.1755324808 \mathrm{e}-2$ & $.232784142 \mathrm{e}-9$ \\
\hline 20 & & -.89361 & $.340409901 \mathrm{e}-2$ & $.456549799 \mathrm{e}-8$ \\
\hline 21 & & -.181 & $-.2741804 \mathrm{e}-2$ & $.7309216472 \mathrm{e}-7$ \\
\hline 22 & & & $.353555 \mathrm{e}-3$ & $.9368893764 \mathrm{e}-6$ \\
\hline 23 & & & .117734 & $.9348804928 \mathrm{e}-5$ \\
\hline 24 & & & $.20714 \mathrm{e}-1$ & $.69517414 \mathrm{e}-4$ \\
\hline 25 & & & -.9671 & $.356576507 \mathrm{e}-3$ \\
\hline 26 & & & -.284 & $.1059852 \mathrm{e}-2$ \\
\hline 27 & & & 1.3 & $.8242527 \mathrm{e}-3$ \\
\hline 28 & & & -1. & $-.206921 \mathrm{e}-2$ \\
\hline 29 & & & & $.181031 \mathrm{e}-1$ \\
\hline 30 & & & & $.862815 \mathrm{e}-1$ \\
\hline 31 & & & & -.14025 \\
\hline 32 & & & & -.91619 \\
\hline 33 & & & & -.942 \\
\hline 34 & & & & $-.153 \mathrm{e}-1$ \\
\hline 35 & & & & $-.3 ?$ \\
\hline 36 & & & & $?$ \\
\hline
\end{tabular}

TABLE 5.2.1. Coefficients of $Q_{k}(x)=d_{0}(k) x^{k(k+1) / 2}+d_{1}(k) x^{k(k+1) / 2}+\cdots$, for $k=1 \quad 8$ odd twists $d<0$ 


\begin{tabular}{|c|c|c|c|c|}
\hline$r$ & $e_{r}(1)$ & $e_{r}(2)$ & $e_{r}(3)$ & $e_{r}(4)$ \\
\hline 0 & .3522211004995828 & $.1238375103096 \mathrm{e}-1$ & $.1528376099282 \mathrm{e}-4$ & $.31582683324433 \mathrm{e}-9$ \\
\hline 1 & -.4889851881547 & $.6403273133043 \mathrm{e}-1$ & $.60873553227400 \mathrm{e}-3$ & $.40700020814812 \mathrm{e}-7$ \\
\hline 2 & & -.403098546303 & $.51895362572218 \mathrm{e}-2$ & $.19610356347280 \mathrm{e}-5$ \\
\hline 3 & & .878472325297 & -.20704166961612e-1 & $.4187933734219 \mathrm{e}-4$ \\
\hline 4 & & & $-.4836560144296 \mathrm{e}-1$ & $.32338329823195 \mathrm{e}-3$ \\
\hline 5 & & & .6305676273171 & $-.7264209058150 \mathrm{e}-3$ \\
\hline 6 & & & -1.23114954368 & $-.97413031149 \mathrm{e}-2$ \\
\hline 7 & & & & $.6254058547 \mathrm{e}-1$ \\
\hline 8 & & & & $.533803934 \mathrm{e}-1$ \\
\hline 9 & & & & -1.125788 \\
\hline 10 & & & & 2.125417 \\
\hline$r$ & $e_{r}(5)$ & $e_{r}(6)$ & $e_{r}(7)$ & $e_{r}(8)$ \\
\hline 0 & $.671251761107 \mathrm{e}-16$ & $.1036004645427 \mathrm{e}-24$ & $.886492719 \mathrm{e}-36$ & $.337201 \mathrm{e}-49$ \\
\hline 1 & $.2024913313373 \mathrm{e}-13$ & $.6113326104277 \mathrm{e}-22$ & $.91146378 \mathrm{e}-33$ & $.556982629 \mathrm{e}-46$ \\
\hline 2 & $.261100345555 \mathrm{e}-11$ & $.16322243213252 \mathrm{e}-19$ & $.437008961 \mathrm{e}-30$ & $.43686422 \mathrm{e}-43$ \\
\hline 3 & $.187088892376 \mathrm{e}-9$ & $.2605311255687 \mathrm{e}-17$ & $.1297363095 \mathrm{e}-27$ & $.216465856 \mathrm{e}-40$ \\
\hline 4 & $.8086250862418 \mathrm{e}-8$ & $.2766415183453 \mathrm{e}-15$ & $.2670392090 \mathrm{e}-25$ & $.7604817313 \mathrm{e}-38$ \\
\hline 5 & $.2126496335545 \mathrm{e}-6$ & $.2056437432502 \mathrm{e}-13$ & $.404346681 \mathrm{e}-23$ & $.201532781 \mathrm{e}-35$ \\
\hline 6 & $.319415704903 \mathrm{e}-5$ & $.10957094998959 \mathrm{e}-11$ & $.46631481394 \mathrm{e}-21$ & $.418459324 \mathrm{e}-33$ \\
\hline 7 & $.21201987479 \mathrm{e}-4$ & .42061728711797e-10 & $.41831543311 \mathrm{e}-19$ & $.698046515 \mathrm{e}-31$ \\
\hline 8 & $-.33900555230 \mathrm{e}-4$ & $.11491097182922 \mathrm{e}-8$ & $.29548572643 \mathrm{e}-17$ & $.951665168 \mathrm{e}-29$ \\
\hline 9 & $-.775061385 \mathrm{e}-3$ & $.21545094604323 \mathrm{e}-7$ & $.1652770327 \mathrm{e}-15$ & $.1073015400 \mathrm{e}-26$ \\
\hline 10 & $.333997849 \mathrm{e}-2$ & $.25433712247032 \mathrm{e}-6$ & $.73192383650 \mathrm{e}-14$ & $.1008662234 \mathrm{e}-24$ \\
\hline 11 & $.22204682 \mathrm{e}-1$ & $.1448397731463 \mathrm{e}-5$ & $.25506469557 \mathrm{e}-12$ & $.7945270901 \mathrm{e}-23$ \\
\hline 12 & -.1538433 & $-.2179868777201 \mathrm{e}-5$ & $.6901276286 \mathrm{e}-11$ & $.5257922143 \mathrm{e}-21$ \\
\hline 13 & $-.19794 \mathrm{e}-1$ & $-.54298634893 \mathrm{e}-4$ & $.141485467 \mathrm{e}-9$ & $.2924082555 \mathrm{e}-19$ \\
\hline 14 & 2.01541 & $.1698771341 \mathrm{e}-3$ & $.210241720 \mathrm{e}-8$ & $.1363867915 \mathrm{e}-17$ \\
\hline 15 & -4.451 & $.22887524 \mathrm{e}-2$ & $.20651382 \mathrm{e}-7$ & $.5311448709 \mathrm{e}-16$ \\
\hline 16 & & -.1042e-1 & $.101650951 \mathrm{e}-6$ & $.1714154659 \mathrm{e}-14$ \\
\hline 17 & & $-.4339429 \mathrm{e}-1$ & $-.16979129 \mathrm{e}-6$ & $.453180963 \mathrm{e}-13$ \\
\hline 18 & & .343054 & $-.37367 \mathrm{e}-5$ & $.9644403068 \mathrm{e}-12$ \\
\hline 19 & & -.1947171 & $.97069 \mathrm{e}-5$ & $.160742335 \mathrm{e}-10$ \\
\hline 20 & & -3.16910 & $.18351 \mathrm{e}-3$ & $.200188929 \mathrm{e}-9$ \\
\hline 21 & & 7.31266 & $-.54878 \mathrm{e}-3$ & $.16931900 \mathrm{e}-8$ \\
\hline 22 & & & $-.5621 \mathrm{e}-2$ & $.7257434 \mathrm{e}-8$ \\
\hline 23 & & & $.284 \mathrm{e}-1$ & $-.14329111 \mathrm{e}-7$ \\
\hline 24 & & & $.639 \mathrm{e}-1$ & $-.25913136 \mathrm{e}-6$ \\
\hline 25 & & & -.7 & $.6473933 \mathrm{e}-6$ \\
\hline 26 & & & .86 & $.138673 \mathrm{e}-4$ \\
\hline 27 & & & 5. & $-.2339 \mathrm{e}-4$ \\
\hline 28 & & & $-.1 \mathrm{e} 2$ & $-.48124 \mathrm{e}-3$ \\
\hline 29 & & & & $.162 \mathrm{e}-2$ \\
\hline 30 & & & & $.976 \mathrm{e}-2$ \\
\hline 31 & & & & $-.83 \mathrm{e}-1$ \\
\hline 32 & & & & $-.62 \mathrm{e}-1$ \\
\hline 33 & & & & 2. \\
\hline 34 & & & & -2 . \\
\hline 35 & & & & -9 \\
\hline 36 & & & & $30 . ?$ \\
\hline
\end{tabular}

TABLE 5.2.2. Coefficients of $Q_{k}(x)=e_{0}(k) x^{k(k+1) / 2}+e_{1}(k) x^{k(k+1) / 2}+\cdots$, for $k=1$ 8 even twists $d>0$ 


\begin{tabular}{|c|c|c|c|}
\hline$k$ & reality (5.2.1) & conjecture (5.2.2) & ratio \\
\hline 1 & 1460861.8 & 1460891. & 0.99998 \\
2 & 17225813.8 & 17226897.5 & 0.999937 \\
3 & 316065502.1 & 316107868.6 & 0.999866 \\
4 & 7378585496. & 7380357447.1 & 0.99976 \\
5 & 198754711593.6 & 198809762196.4 & 0.999723 \\
6 & 5876732216291.7 & 5877354317291.3 & 0.999894 \\
7 & 185524225881950. & 185451557119001. & 1.000392 \\
8 & 6149876164696600 & 6141908614344770 & 1.0013 \\
\hline
\end{tabular}

TABLE 5.2.3. Smoothed moment of $L\left(\frac{1}{2}, \chi_{d}\right)$ versus Conjecture 1.5.3, for fundamental discriminants $-1000000<d<0$, and $k=1, \ldots, 8$.

\begin{tabular}{|c|c|c|c|}
\hline$k$ & reality (5.2.1) & conjecture (5.2.2) & ratio \\
\hline 1 & 1144563.5 & 1144535.5 & 1.000024 \\
2 & 9252479.6 & 9252229.9 & 1.000027 \\
3 & 109917867.0 & 109917367.9 & 1.0000045 \\
4 & 1622521963.4 & 1622508843.4 & 1.0000081 \\
5 & 27321430060. & 27320230686. & 1.000043 \\
6 & 501621762060.6 & 501542204848.7 & 1.000159 \\
7 & 9787833470714.1 & 9783848274459.6 & 1.000407 \\
8 & 199831160877919 & 199664775232854 & 1.000833 \\
\hline
\end{tabular}

TABLE 5.2.4. Smoothed moment of $L\left(\frac{1}{2}, \chi_{d}\right)$ versus Conjecture 1.5.3, for fundamental discriminants $0<d<1000000$, and $k=1, \ldots, 8$.

\begin{tabular}{|c|c|c|c|c|}
\hline $\mathrm{r}$ & $f_{r}(1)$ & $f_{r}(2)$ & $f_{r}(3)$ & $f_{r}(4)$ \\
\hline 0 & 1.2353 & .3834 & .00804 & .0000058 \\
1 & & 1.850 & .209 & .000444 \\
2 & & & 1.57 & .0132 \\
3 & & & 2.85 & .1919 \\
4 & & & & 1.381 \\
5 & & & & 4.41 \\
6 & & & & 4.3 \\
\hline
\end{tabular}

TABLE 5.3.1. Coefficients of $\Upsilon_{k}(x)=f_{0}(k) x^{k(k-1) / 2}+f_{1}(k) x^{k(k-1) / 2-1}+\ldots$, for $\mathrm{k}=1,2,3,4$.

\begin{tabular}{|c|c|c|c|}
\hline$k$ & left side (5.3.8) & right side (5.3.8) & ratio \\
\hline 1 & 14628043.5 & 14628305. & 0.99998 \\
2 & 100242348.8 & 100263216. & 0.9998 \\
3 & 1584067116.8 & 1587623419. & 0.998 \\
4 & 41674900434.9 & 41989559937. & 0.993 \\
\hline
\end{tabular}

TABLE 5.3.2. Moments of $L_{11}\left(\frac{1}{2}, \chi_{d}\right)$ versus their conjectured values, for fundamental discriminants $-85,000,000<d<0, d=2,6,7,8,10 \bmod 11$, and $k=1, \ldots, 4$. The data agree with our conjectures to the accuracy to which we have computed the moment polynomials $\Upsilon_{k}$. 

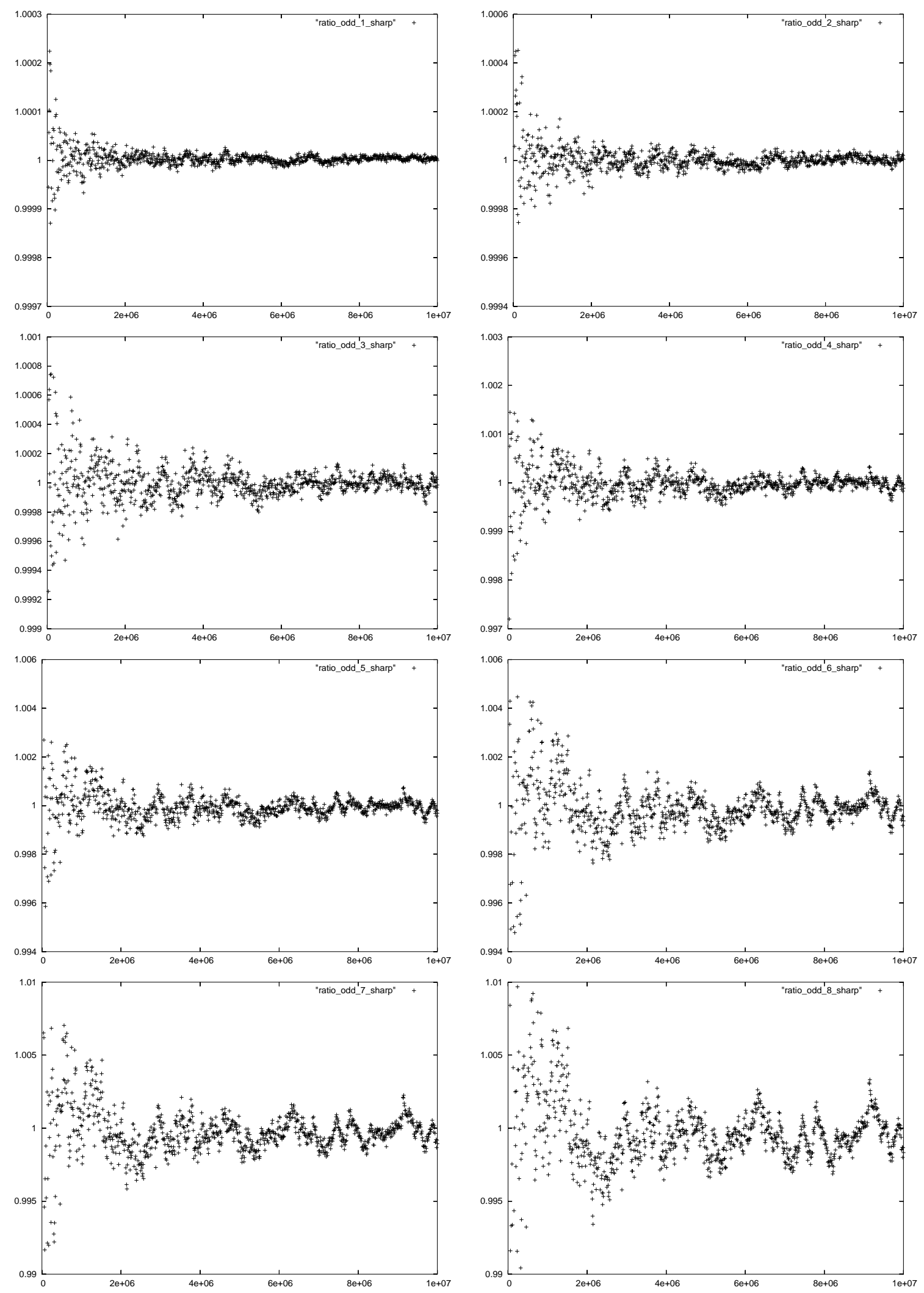

Figure 1. Horizontal axis in each graph is $X$. These graphs depict the first eight moments, sharp cutoff, of $L\left(1 / 2, \chi_{d}\right),-X \leq d<0$ divided by the conjectured value, sampled at $X=10000,20000, \ldots, 10^{7}$. One sees the graphs fluctuating above and below one. Notice that the vertical scale varies from 

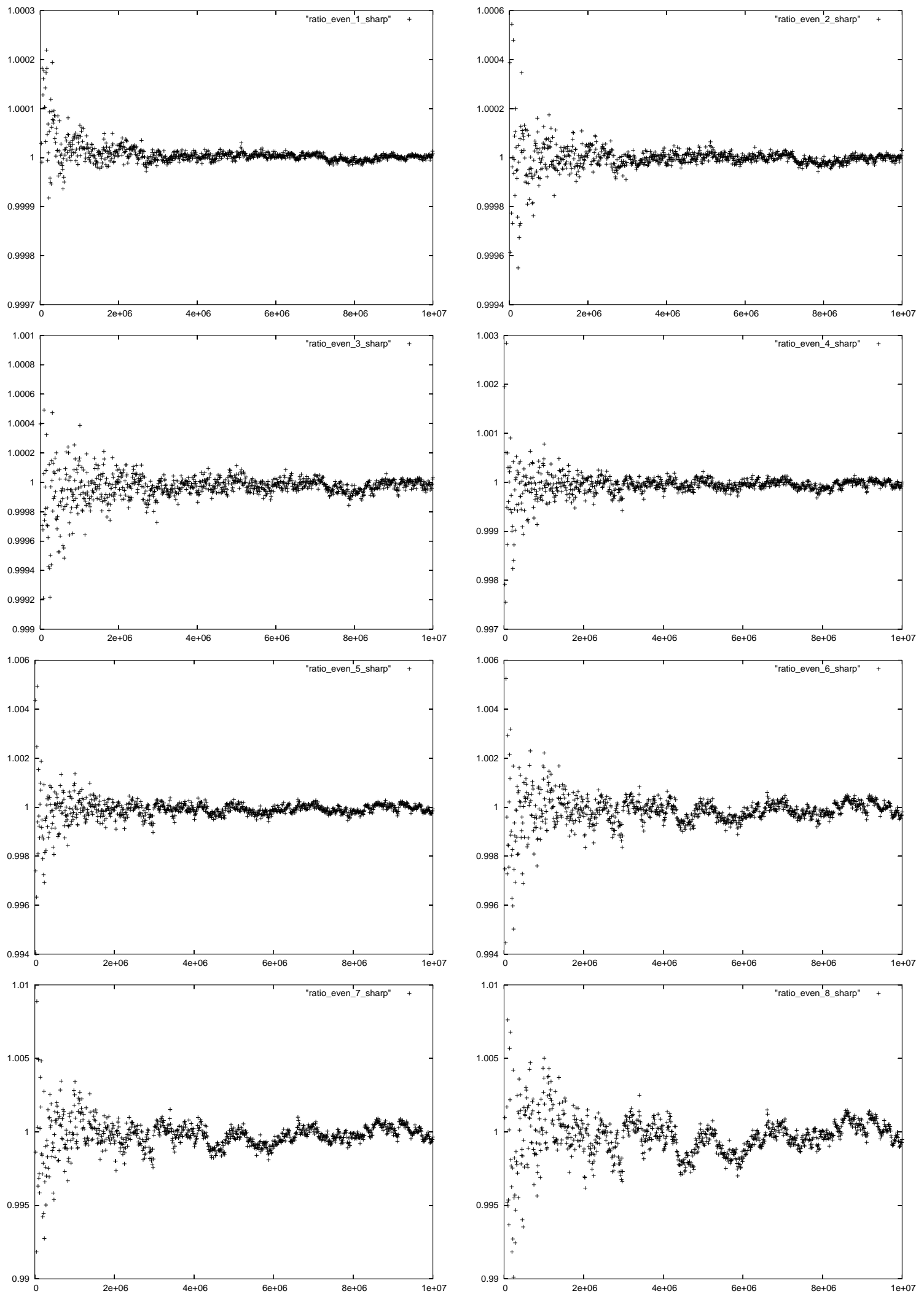

Figure 2. Same as the previous fingure, but for $0<d \leq X$. 